University of Louisville

ThinkIR: The University of Louisville's Institutional Repository

$12-2018$

\title{
The regional distribution and molecular interactions of phospholipids and glucose in mammalian vitreous humors.
}

\author{
Abigail Nicole Schnepf \\ University of Louisville
}

Follow this and additional works at: https://ir.library.louisville.edu/etd

Part of the Analytical Chemistry Commons

\section{Recommended Citation \\ Schnepf, Abigail Nicole, "The regional distribution and molecular interactions of phospholipids and glucose in mammalian vitreous humors." (2018). Electronic Theses and Dissertations. Paper 3136. https://doi.org/10.18297/etd/3136}

This Doctoral Dissertation is brought to you for free and open access by ThinkIR: The University of Louisville's Institutional Repository. It has been accepted for inclusion in Electronic Theses and Dissertations by an authorized administrator of ThinkIR: The University of Louisville's Institutional Repository. This title appears here courtesy of the author, who has retained all other copyrights. For more information, please contact thinkir@louisville.edu. 
THE REGIONAL DISTRIBUTION AND MOLECULAR INTERACTIONS OF PHOSPHOLIPIDS AND GLUCOSE IN MAMMALIAN VITREOUS HUMORS

\author{
By \\ Abigail Nicole Schnepf \\ B.S., Bellarmine University, 2008 \\ M.A., University of Louisville, 2016

\begin{abstract}
A Dissertation
Submitted to the Faculty of the College of Arts and Sciences of the University of Louisville In Part Fulfillment of the Requirements

For the Degree of
\end{abstract}

Doctor of Philosophy in Chemistry

Department of Chemistry

University of Louisville

Louisville, Kentucky

December 2018 

THE REGIONAL DISTRIBUTION AND MOLECULAR INTERACTIONS OF PHOSPHOLIPIDS AND GLUCOSE IN MAMMALIAN VITREOUS HUMORS

By

Abigail Nicole Schnepf

B.S., Bellarmine University, 2008

M.S., University of Louisville, 2016

A Dissertation Approved on

July $31^{\text {st }}, 2018$

By the following Dissertation Committee:

Dissertation Director

Dr. M. Cecilia Yappert

Dr. Douglas Borchman

Dr. Muriel Maurer

Dr. Francis Zamborini 


\section{ACKNOWLEDGEMENTS}

I would first and foremost like to thank my research advisor, Dr. M. Cecilia Yappert. Her love and passion for science along with her guidance and support has helped me succeed throughout my graduate school career by giving me the drive to always better myself through my work. I would also like to thank our collaborator, Dr. Douglas Borchman, who always provided ideas, support, and laughter whenever needed. I would also like to thank my other committee members, Drs. Muriel Maurer and Francis Zamborini, who have supported me along the way with guidance and advice for my project. I would also like to thank my lab members who are always willing to go the extra length to help with anything that might be needed. I wish them nothing but success in their future endeavors. I would also like to thank the other faculty members at the University of Louisville for always wanting to help in anyway they can.

This project would not have been possible without the financial support and guidance of the Chemistry department at University of Louisville. I would also like to thank the Research Initiative of University of Louisville for their grant to provide financial support.

Most importantly I would like to thank my family and friends. My mother and father, Kristi and Dave, have always given me the love and support to accomplish any goal I have ever set for myself. I would also like to thank my brother and sister, Jacob and Rachel, for always being there for me. I would specifically like to thank my best friend, Kendall, and fiancé, Patrick, who have been with me every step of the way 
throughout my graduate school career. I will forever be thankful for their love and friendship along the way and appreciate them always being there for me no matter the circumstances. I would also like to thank the rest of my friends and family for all of their support over the years. 


\begin{abstract}
THE REGIONAL DISTRIBUTION AND MOLECULAR INTERACTIONS OF PHOSPHOLIPIDS AND GLUCOSE IN MAMMALIAN VITREOUS HUMORS
\end{abstract}

Abigail Nicole Schnepf

July $31^{\text {st }}, 2018$

The vitreous humor $(\mathrm{VH})$ is located in between the lens and the retina. It is composed of $98 \%$ water, hyaluronan (HA), collagen, proteins, phospholipids (PLs), and other metabolites. With aging, the VH undergoes liquefaction, a process that causes the gel-like structure of the VH to turn into a liquid and can lead to serious ocular diseases, including retinal detachment, vitreal detachment, and macular hole formation. The liquefaction process is expedited in diabetic VHs. This project focuses on understanding the molecular changes that lead to liquefaction and the reasons for which this process is accelerated with diabetes.

Matrix-assisted laser desorption ionization/mass spectrometry (MALDI/MS) and nuclear magnetic resonance (NMR) spectroscopy were applied for in vitro, ex situ, and model studies of VHs to identify and quantify several components of the VH network and explore their interactions. In-vitro and ex-situ methods were optimized and conducted on porcine and human VHs. The mammalian species produced similar compositional trends. MALDI/MS showed that PLs were most abundant in the posterior region (closest to the retina) followed by the anterior (closest to the lens) and then the central regions. Diabetic and non-diabetic VHs regional distribution of glucose was compared. Glucose was 
present in significant higher levels (three-fold) in diabetic VHs compared to non-diabetic ones of similar age. The levels of glucose in the diabetic $\mathrm{VH}$ were highest in the posterior followed by the anterior and central regions. This trend follows that observed for the PLs. Model studies were performed to explore the interaction(s) of vitreal components and hyaluronan (HA) using MALDI/MS and NMR. The studies indicate interactions between both the headgroups of PLs and the carboxylate groups of HA as well as that between the acyl tails on PC with the hydrophobic regions of HA. Such interactions are proposed to disrupt the H-bonding network of HA and contribute to liquefaction of the VH. Future studies will focus on age-dependent studies of human VHs as well as the analysis of fresh human VHs following vitrectomy surgery. 
TABLE OF CONTENTS

PAGE

ACKNOWLEDGEMENTS ............................................................

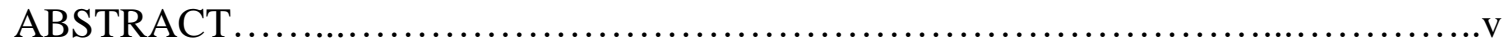

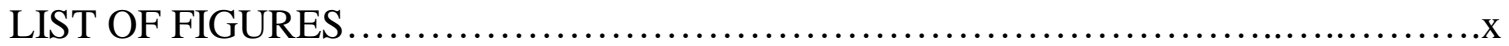

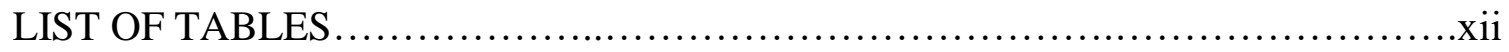

CHAPTER 1. INTRODUCTION AND BACKGROUND ...........................

VITREAL COMPONENTS ...............................................

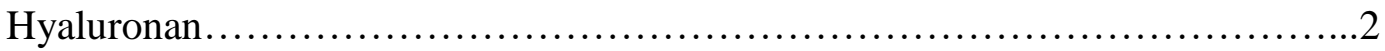

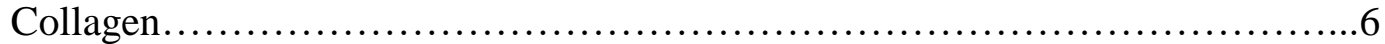

PLs, glucose and other components........................................10

DEVELOPMENT OF THE VITREOUS HUMOR............................12

VITREOUS HUMOR FUNCTIONS......................................13

AGEING AND LIQUEFACTION OF THE VH..............................13

Diseases associated with liquefaction..................................16

BARRIERS AROUND THE VH........................................18

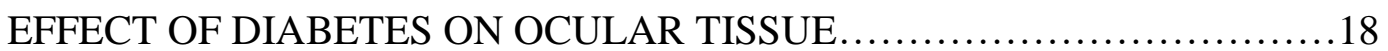

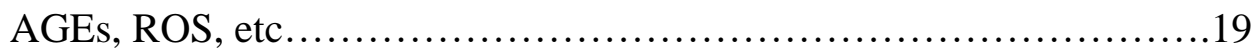

ANALYTICAL TECHNIQUES TO STUDY THE VH.......................20

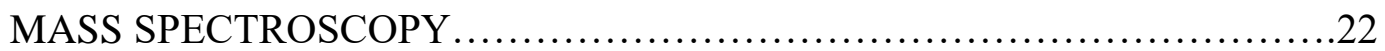

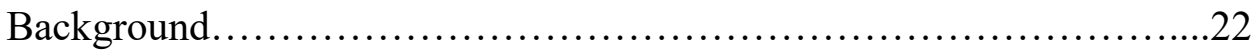

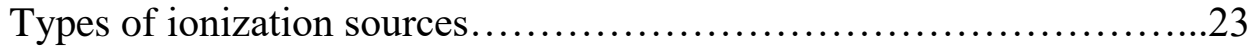

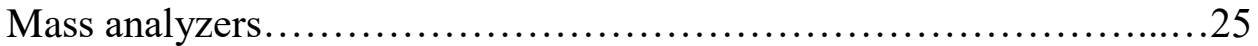

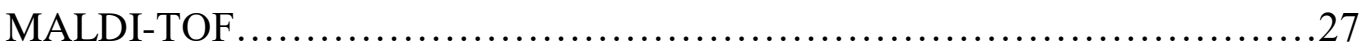

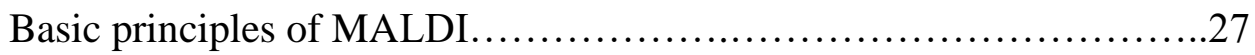

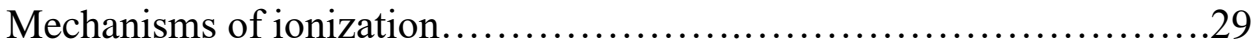

Previous matrices used for MALDI.................................... 30

Basic principles of TOF analyzer........................................ 31

Previous applications of MALDI-TOF ................................34 


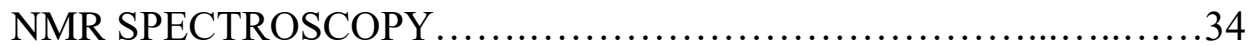

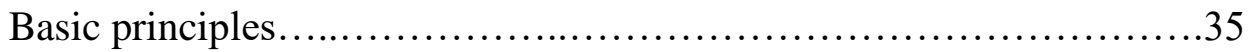

Chemical information obtained from NMR ...........................36

Previous applications of NMR ...................................... 41

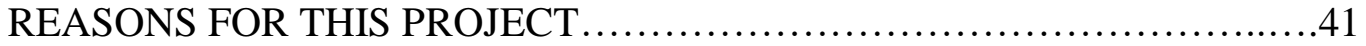

CHAPTER 2. REGIONAL DISTRIBUTION OF PHOSPHOLIPIDS IN PORCINE

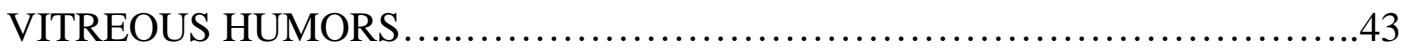

INTRODUCTION ..........................................................

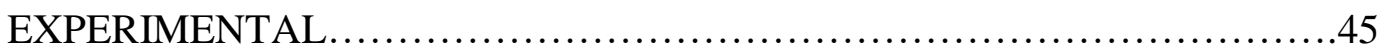

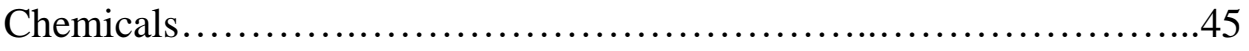

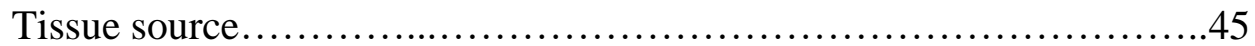

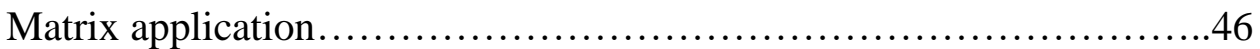

Ex-situ analysis...................................................

In-vitro analysis................................................... 47

Data Acquisition.................................................. 48

RESULTS.............................................................. 48

Experimental optimization........................................ 48

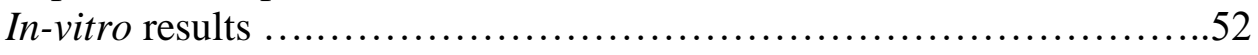

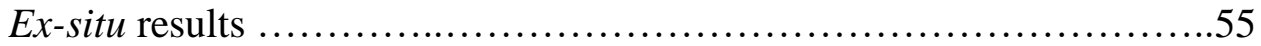

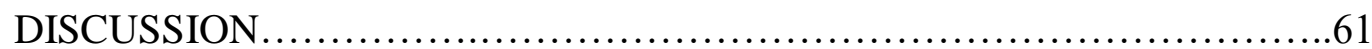

Analytical approaches...........................................61

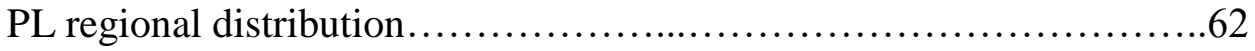

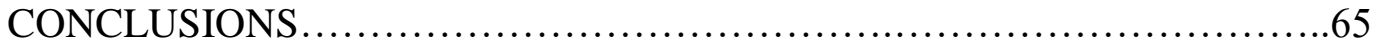

CHAPTER 3. IN-VITRO AND EX-SITU REGIONAL MASS SPECTRAL ANALYSIS OF PHOSPHOLIPIDS AND GLUCOSE IN THE VITREOUS HUMOR FROM DIABETIC AND NON-DIABETIC DONORS..............................67

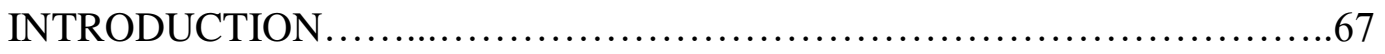

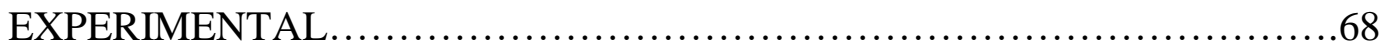

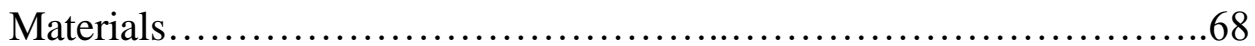

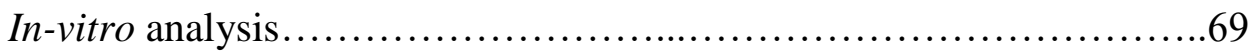

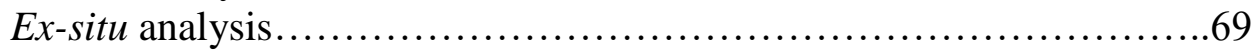

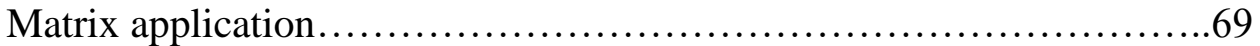

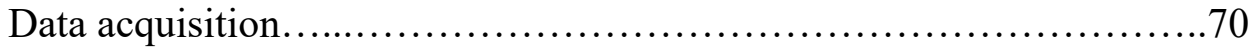

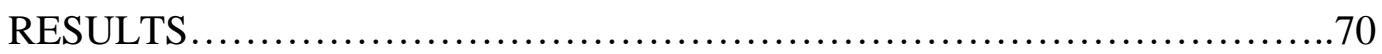

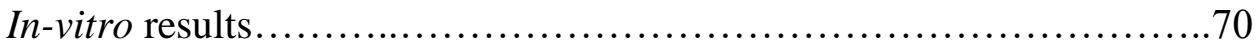


Ex-situ results

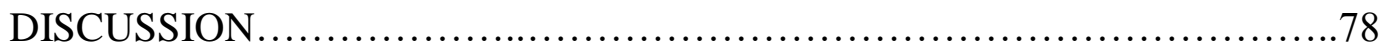

CHAPTER 4. INTERACTIONS OF HYALURONAN WITH GLUCOSE AND PHOSPHOLIPIDS DETERMINED USING MALDI/MS AND NMR...............82

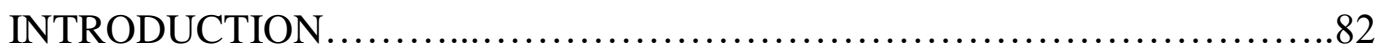

EXPERIMENTAL ........................................................ 85

Chemicals........................................................ 85

Matrix preparation.............................................85

Model studies........................................................

Porcine VH analysis........................................... 86

Data acquisition................................................ 86

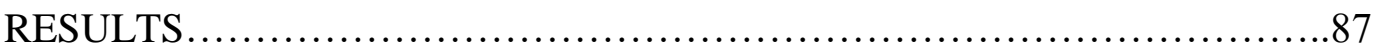

Changes in relative ionization efficiency for PLs in presence of HA....87

Changes in NMR spectral features for PLs in presence of HA...........88

Changes in RIE for glucose in presence of HA.....................92

Changes in NMR spectral features for glucose in presence of HA.........92

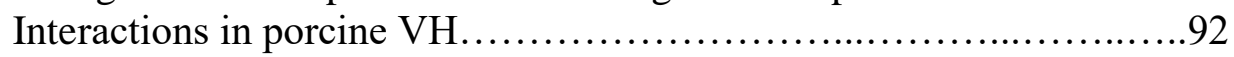

DISCUSSION......................................................... 96

Model studies of phospholipids and hyaluronan......................96

Model studies of glucose and hyaluronan...........................99

VH tissue results................................................. 100

Model and tissue comparisons.................................101

CONCLUSIONS......................................................... 101

CHAPTER 5. CONCLUSIONS AND FUTURE DIRECTIONS ......................103

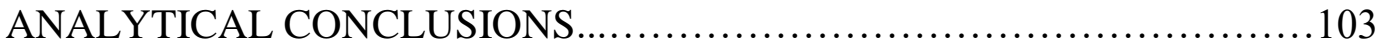

Tissue sectioning.................................................. 103

Matrix choices.................................................... 104

Impact of molecular interactions on RIE..........................104

PHYSIOLOGICAL CONCLUSIONS .................................... 105

Regional distribution of phospholipids and glucose in VHs............105

Impact of diabetes in levels of PLs and glucose in human VHs..........105

INTERACTIONS OF HYALURONAN WITH PLs AND GLUCOSE.........106

FUTURE STUDIES ................................................... 107

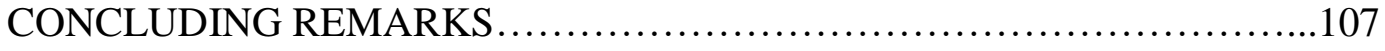




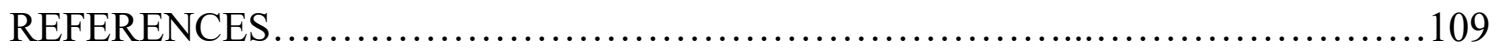

APPENDIX ............................................................ 120

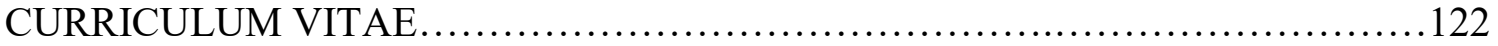




\section{LIST OF FIGURES}

FIGURE

PAGE

1-1. Components of the eye and the distribution of HA and collagen.................2

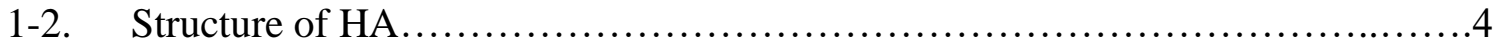

1-3. Proposed interaction of collagen and HA in the VH.........................

1-4. Proposed interactions of heterotopic collagen fibrils in the VH...............8

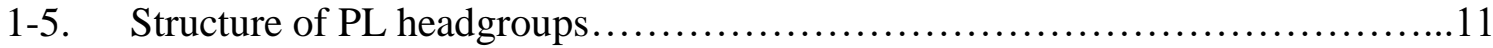

1-6. Development of the VH............................................

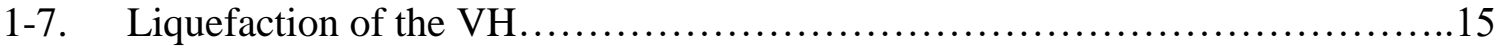

1-8. Principle of MALDI................................................ 27

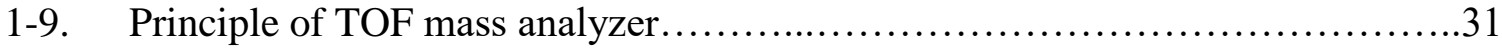

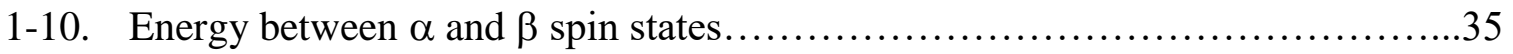

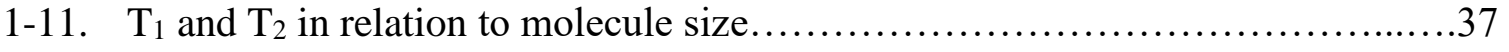

2-1. PNA dissolved in $\mathrm{MeOH}, \mathrm{CHCl}_{3}, \mathrm{H}_{2} \mathrm{O}: \mathrm{MeOH}(1: 1)$, and $\mathrm{CHCl}_{3}: \mathrm{MeOH} \ldots . . . . .48$

2-2. PNA dissolved in a) plastic Eppendorf tube and b) glass vial.................49

2-3. Extraction of PLs using $\mathrm{MeOH}: \mathrm{CHCl}_{3}(1: 2)$ (a) and $\mathrm{MeOH}$ only (b) on the lens, $\mathrm{VH}$, and retina......................................................52

2-4. B\&D extraction of the a) anterior region of the $\mathrm{VH}, \mathrm{b}$ ) central region of the $\mathrm{VH}, \mathrm{c}$ ) posterior region of the $\mathrm{VH}, \mathrm{d}$ ) lens, and $\mathrm{f}$ ) retina from e) frozen porcine eye......53

2-5. Imprint of porcine $\mathrm{VH}$ from the a) posterior $\mathrm{VH}$, b) central $\mathrm{VH}$,

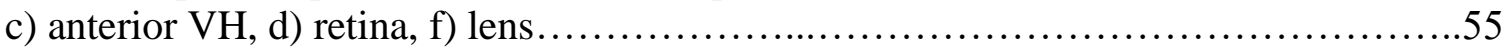

2-6. Negative mode of the a) retina and b) lens................................57 
3-1. MALDI-TOF spectra obtained in-vitro from extracts of VHd and VHnd.......68

3-2. Comparison of PL composition in the regional analysis of diabetic and non-diabetic VHs using in-vitro techniques...............................69

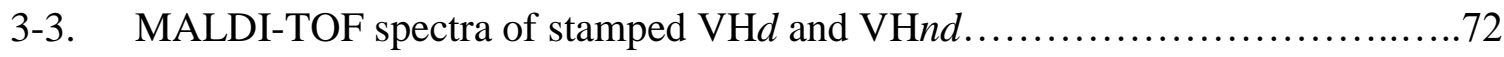

3-4. Comparison of the PL composition in the regional ex-situ analysis of VHd and

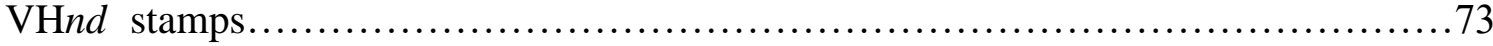

3-5. Relative intensities of glucose in regions of diabetic VH tissue compared to non-diabetic tissue...................................................... 75

4-1. The MALDI spectra of a) PC and b) PC with HA........................... 85

4-2. The relative ionization efficiency of PC in the presence of glucose, HA, and

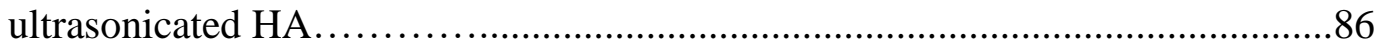

4-3. The NMR spectra of a) PC, b) HA, and c) PC mixed with HA..................88

4-4. MALDI spectra of a) glucose and b) glucose with HA......................89

4-5. The RIE of glucose in the presence of PC, HA, ultrasonicated HA, and PA.....90

4-6. NMR spectra of a) glucose, b) HA, and c) mixture of glucose and HA. .........91

4-7. The MALDI spectra of an extracted porcine VH using the Bligh and Dyer method a) without sonication and b) with ultrasonication...........................93

4-8. The relative intensities of $\mathrm{PC}(32: 0)$ and $\mathrm{PC}(34: 1)$ normalized with DMPC from porcine VHs.................................................94 


\section{LIST OF TABLES}

TABLE

PAGE

1-1. Summary of PL species detected in porcine tissue using in-vitro

B\&D extraction technique

1-2. Summary of PLs detected in porcine tissue using ex-situ stamp technique........58

1-3. Summary of PLs found in the retina and lens of various mammals ............62 


\section{CHAPTER 1 \\ INTRODUCTION AND BACKGROUND}

This chapter provides crucial background information on the vitreous humor (VH) and the analytical methodologies used to complete this project. The first section of the chapter focuses on vitreal components, functions, liquefaction, migration, and interaction of components affecting the biomatrix. The second part of this chapter describes the analytical techniques, specifically matrix-assisted laser desorption ionization (MALDI) mass spectrometry (MS) and nuclear magnetic resonance (NMR) spectroscopy, that we used to study the mammalian VHs. Chapters 2 and 3 present the application of these methodologies to find the regional distribution of phospholipids (PLs) and glucose the VHs of porcine and human eyes. Chapter 4 discusses the interactions of vitreal components with PLs and glucose and their implications in VH liquefaction. Finally, chapter 5 addresses the main findings of these projects and the direction of future studies.

\section{VITREAL COMPONENTS}

Light is directed into the eye and is focused by the cornea $(75 \%$ of focusing power of the eye) to the pupil and iris. The iris controls how much light is transmitted through the eye. After the light passes through the pupil and traverses the aqueous humor it is focused onto the retina by the lens ( $25 \%$ of the focusing power of the eye). The retina 
uses photorecepters to convert light to electrical signals. The optic nerve carries the electrical signals to the brain that allows the light to be processed as an image. Figure 1-1 shows the anatomical components of the human eye. Occupying about $80 \%$ of the ocular volume is the vitreous humor $(\mathrm{VH})$, which is located in between the lens and the retina. The VH needs to be transparent to allow light to pass through without being scattered.

The $\mathrm{VH}$ is made up of over $98 \%$ water that hydrates a network of hyaluronan and collagen in which proteins, phospholipids, and other metabolites are found. ${ }^{1}$ Functions of the $\mathrm{VH}$ and possible causes of liquefaction have been studied previously. Figure 1-1 shows the distribution of collagen and hyaluronan (HA) in the VH. Even though there is a significant amount of information on HA and collagen, the interactions with each other and with other components in the $\mathrm{VH}$, such as phospholipids and glucose have yet to be explored.

\section{Hyaluronan}

HA was first detected in 1934 by Karl Meyer and John Palmer from bovine VH and the structure of HA was determined in $1950 .{ }^{3,4}$ In the $1950 \mathrm{~s}$, HA was first used as a vitreal replacement during ocular surgery. ${ }^{5}$ Other applications of HA include wound healing, cosmetics, and prevention of blood coagulation using HA derivatives for cardiovasculature applications. ${ }^{6}$ HA is a type of glycosaminoglycan (GAG), a polysaccharide with repeating units. When a GAG is attached to a protein it is known as a proteoglycan. Besides HA, other types of GAGs found in the $\mathrm{VH}$ are chondroitin sulfate proteoglycans, which include versican and type IX collagen. Collagen type IX and versican are synthesized in the Golgi apparatus and HA is synthesized at the plasma membranes. ${ }^{7}$ 


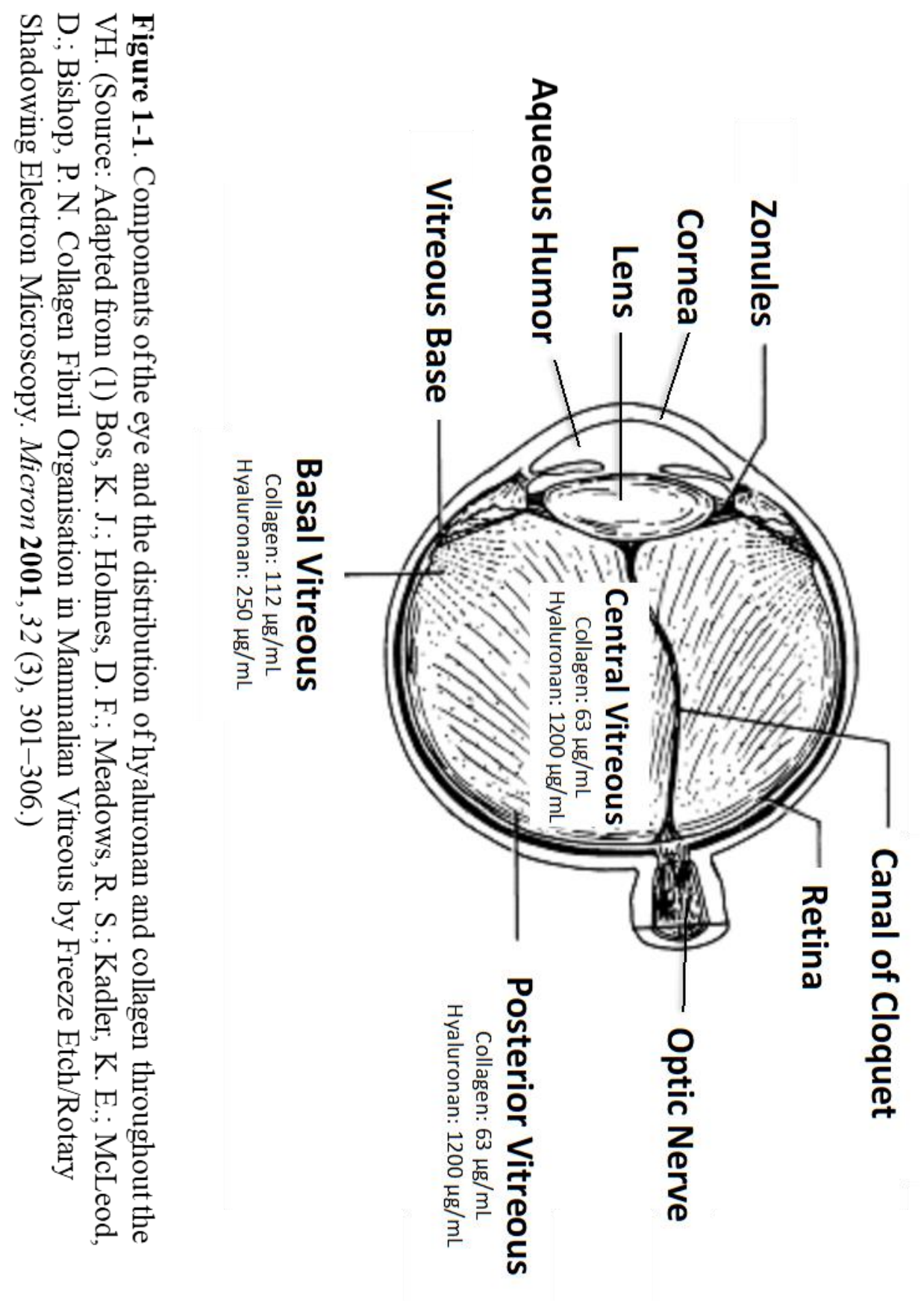


HA is composed of polyanionic saccharides which are made up of glucuronic acid (GlcA), and an amino sugar, glucosamine (N-GlcNA) linked via a glycosidic bond (Fig. 1-2). ${ }^{3}$ GlcA and GlcNA form the repeating unit of a long unbranched polysaccharide. The length of the HA chain varies by species. For example, a rabbit VH has 2000-3000 kDa HA strands but bovine HA strands are shorter at $500-800 \mathrm{kDa} .{ }^{8}$ Along the polysaccharide chains, hydrogen bonds cause the strand to twist and form a helical 'ribbon' with hydrophobic and hydrophilic regions between the $\beta 1-3$ and the $\beta 1-4$ linkages. ${ }^{9,10}$ This feature allows HA to interact with other molecules and HA strands. The ability to interact with molecules gives HA its viscoelastic properties. One disaccharide unit of HA can interact with 15 water molecules. ${ }^{11}$ Polymeric HA can interact with water to increase its molecular weight up to 1000 fold. ${ }^{12}$ Molecular dynamics show that water molecules force the disaccharide units to form stronger interactions causing the glycosidic linkages to stiffen as well as form water bridges forming hydrogen bonded complexes. ${ }^{13}$ HA interacts with PLs as well. The flexibility of HA changes upon interacting with phosphatidylcholines (PCs) based on broadening of the methyl protons on N-GlcNA of HA. The PC tails possibly interact with the hydrophobic patches of HA based on this NMR data in addition to data from gel permeation chromatography and multi-angle laserlight scattering. ${ }^{14}$ The organization of PC is affected by the molecular weight of HA. PC aggregates around the HA strands. ${ }^{15} \mathrm{HA}$ also interacts with collagen type VI. ${ }^{16}$ However, the HA-collagen interactions are weak and break when exposed to high salt concentrations. ${ }^{17}$ Collagen type VI consists of 3 alpha chains so the N-terminus on the alpha-3 chain of the collagen can interact with HA or type II collagen. ${ }^{18} \mathrm{HA}$ also has a 


\section{D-Glucuronic Acid N-Acetylglucosamine}

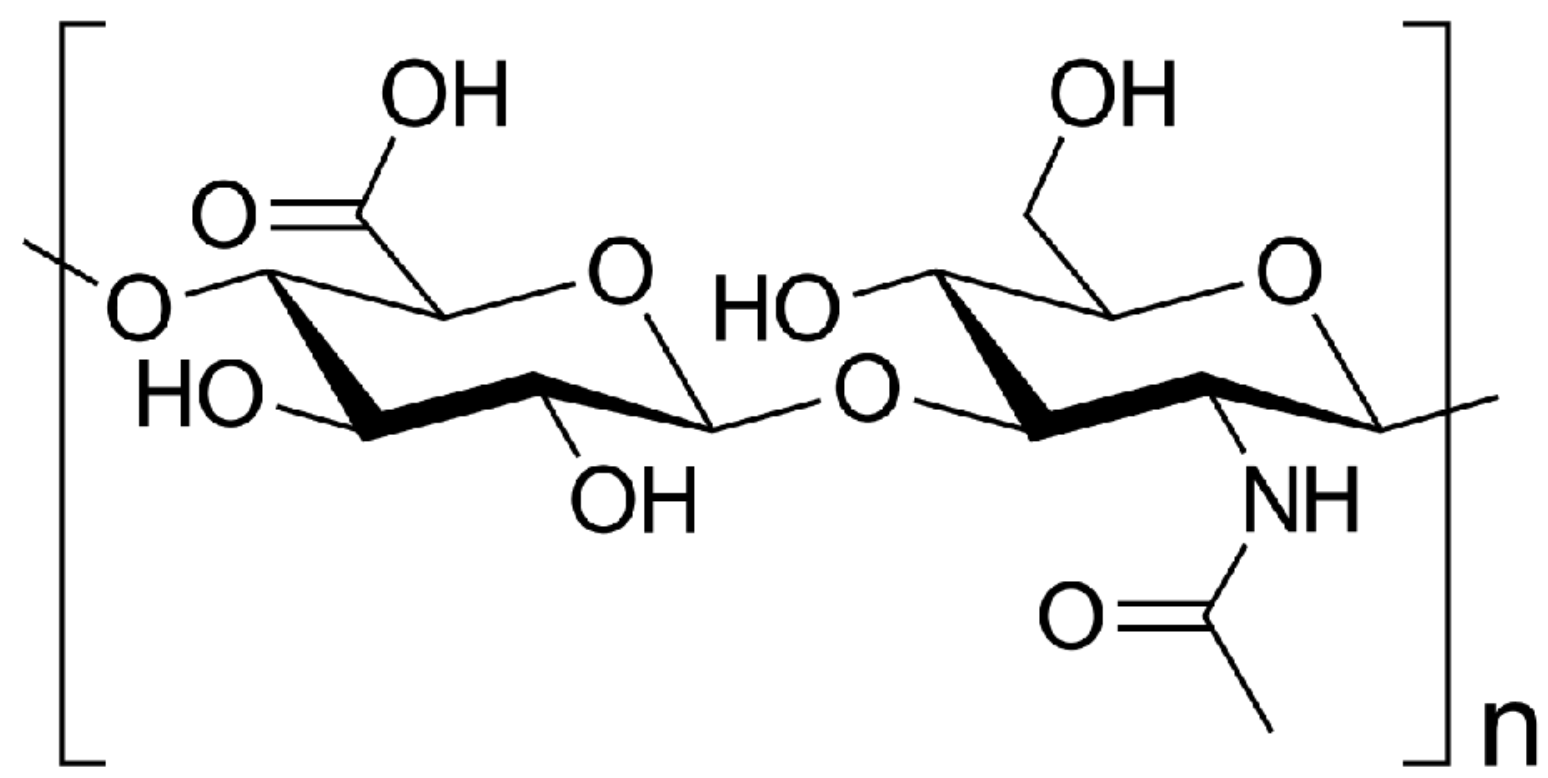

Figure 1-2: Structure of hyaluronan. Disaccharide made up of D-glucuronic acid and Nacetylglucosamine. 
high affinity to other proteoglycans, cell membranes, and cell receptors such as CD-44, a cell adhesion glycoprotein. ${ }^{19}$

It has been confirmed using circular dichroism that there is a conformational change of HA depending on its physical state (gel or liquid). ${ }^{20}$ At a high viscosity, the ${ }^{13} \mathrm{C}$ NMR resonance $\mathrm{C}=\mathrm{O}$ of $\mathrm{HA}$ broadens but when the viscosity is reduced the peak sharpens due to hydrogen bonds breaking and allowing the acetamido groups to rotate freely. ${ }^{21}$ Oxygen in the singlet state can also change the conformation of $\mathrm{HA} .{ }^{20}$ When the $\mathrm{pH}$ is at the cellular level, around 7, the carboxyl groups of HA are all ionized giving it an overall negative charge. ${ }^{10}$

Depending on the size of the strand, HA can either function as a space filler with long strands or cause inflammation and stimulate an immune response with shorter strands. ${ }^{7}$ HA slows down diffusion of molecules through steric hindrance. ${ }^{16} \mathrm{HA}$ is also important in water homeostasis, cell signaling, angiogenesis, axonal growth, tumor progression, cell proliferation, inhibiting lymphocyte stimulation, macrophage phagocytosis, and prostaglandin synthesis. ${ }^{20}$ The lowest concentration of HA is in the anterior region of the $\mathrm{VH}$ and the concentration increases towards the posterior region. ${ }^{20}$

\section{Collagen}

The focus of this project is to address the roles that HA and PLs as well as their interactions play in liquefaction. However, it should be noted that collagen is also an important component of the $\mathrm{VH}$ and its participation in liquefaction will be investigated in future studies. The proposed interaction between collagen and HA is seen in figure 1-3. 


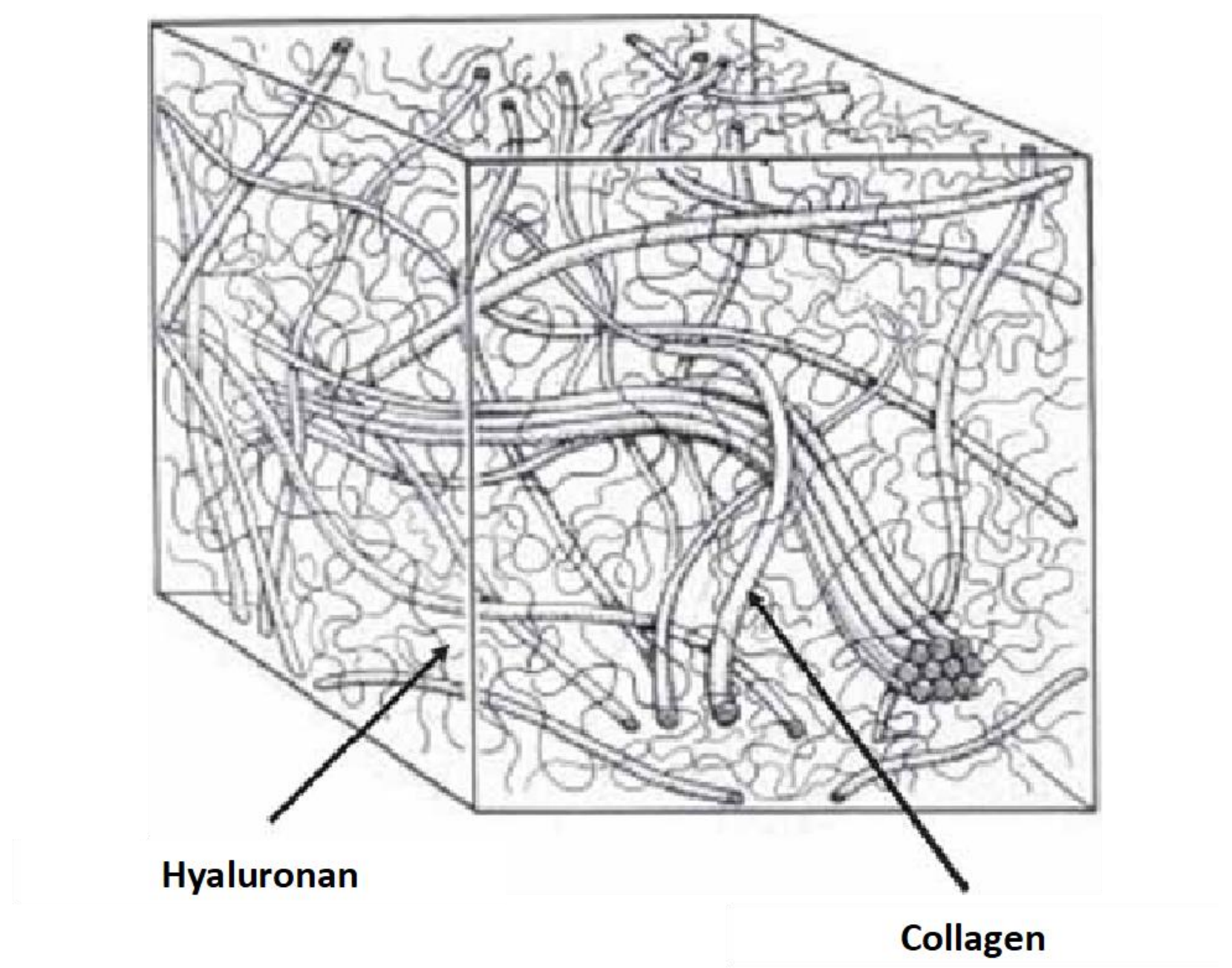

Figure 1-3. Proposed interaction of hyaluronan and collagen in the VH. (Source: Adapted from Gandorfer, Arnd. (2009). Objective of Pharmacologic Vitreolysis. Developments in ophthalmology. 44. 1-6.) 
Collagen forms a left-handed triple helix with 3 amino acid residues per turn. It usually contains the amino acid pattern of $(\mathrm{gly}-\mathrm{x}-\mathrm{y})_{\mathrm{n}}{ }^{22}$ There are many types of collagen that have been discovered but the most abundant in the VH are types II, IX, and V/XI. The structures are seen in figure 1-4. Collagen is initially in a form known as procollagen. $\mathrm{N}$-proteinase and $\mathrm{C}$-proteinase cleave the $\mathrm{N}$ - and $\mathrm{C}$ - terminals, which are non-collagenous and link everything together. This allows the procollagen to form collagen fibrils. ${ }^{23}$ The various types of collagen have different functions. Type II collagen acts like the skeleton because it provides strength and stability to the collagen network. ${ }^{24}$ Type II is made up of 3 identical chains known as $\alpha 1(\mathrm{I})$. The NC3 domain of type II has a bend which allows the binding of its positively charged N-terminus with other molecules such as negatively charged proteoglycans. ${ }^{24}$ Types IX and V/XI are heterotrimers. ${ }^{24} \mathrm{IX}$ is made up of $\alpha 1(\mathrm{IX}) \alpha 2(\mathrm{IX}) \alpha 3(\mathrm{IX})$ domains and are located between 4 non-collagenous domains thus making it a proteoglycan. ${ }^{7,25}$ Type V/XI collagen is a hybrid version of types V and XI. It interacts with type II and makes up the core of the network fibrils. ${ }^{26}$ The composition of this hybrid is made up of $\alpha 1(\mathrm{XI}) \alpha 2(\mathrm{~V})$ domains and is responsible for determining the length and the diameter of the fibrils. Type $\mathrm{V}$ collagen is important in the formation of collagen fibrils. ${ }^{23}$ Type IX is not able to form fibrils on its own so it has to connect itself to other ones. ${ }^{23}$ Type IX runs in the opposite direction of type II collagen and is connected by covalent bonds to the $\mathrm{N}$-terminus of the type II collagen. Chondroitin sulfate chains are also perpendicular to type II collagen which helps prevent type II collagen fibrils from sticking together. ${ }^{24,27}$ Type IX collagen is used for stabilization of the network but is not important for determining the length and diameter of the fibrils. ${ }^{28}$ 


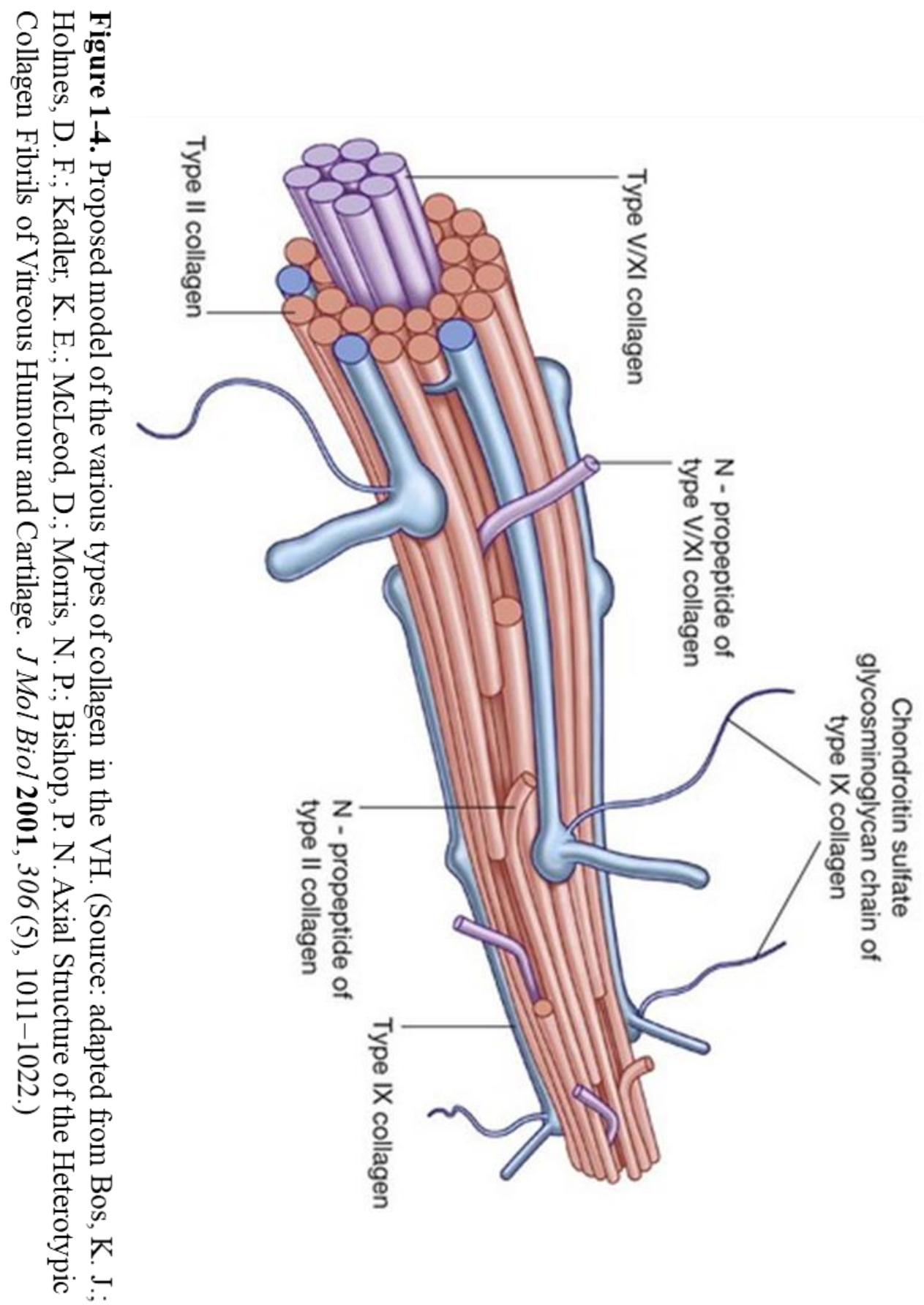


Type IX collagen has a half-life of 11 years and once it starts to break down, type II collagen begins to aggregate due to the collapse of the natural spacers between the fibrils. ${ }^{27}$ Collagen has the highest concentration in the base and posterior regions of the $\mathrm{VH}$, followed by the anterior region of the $\mathrm{VH} .{ }^{29}$ The lowest collagen concentration is found in the central $\mathrm{VH}$. The amount of collagen remains constant throughout the lifetime of the eye since it is not synthesized after the VH is fully developed.

\section{PLs, glucose, and other components of the VH}

Since the VH is made up of $98 \%$ water, the other components are present in very minute quantities. Besides $\mathrm{HA}$ and collagen, other components of the $\mathrm{VH}$ include phospholipids (PLs), cholesterol, chondroitin sulfate proteoglycans, proteins, sodium, potassium, ascorbic acid, glucose, and other metabolites have been found in human VHs. The presence of PLs was reported as early as 1954 by D'Asaro saying that there was minute (if any) PL content in the $\mathrm{VH}^{30}$ Krause reported that the total levels of PLs and cholesterol found in the VH were $0.014 \mathrm{mg} / \mathrm{mL}$ and $0.012 \mathrm{mg} / \mathrm{mL}$, respectively. ${ }^{31}$ The major PLs reported in the $\mathrm{VH}$ were phosphatidylcholine (PC) and phosphatidylethanolamine (PE); sphingomyelin (SM) was reported in lower abundance

(Fig. 1-5). ${ }^{32}$ The fatty acid chains of the PLs were found to be made up of $55 \%$ unsaturated species. The most common unsaturated chains reported were (18:1) and (20:4), where (X:Y) $\mathrm{X}$ is the total number of carbons in the tail and $\mathrm{Y}$ is the number of double bond. The most common saturated tails are of (16:0) and (18:0). ${ }^{33}$

A type of chondroitin sulfate proteoglycan present in the $\mathrm{VH}$ is versican. It is a high molecular weight protein that has been shown to aggregate with HA. ${ }^{34}$ The versican amino terminus can interact with HA or a protein and the carboxyl-terminus can interact 


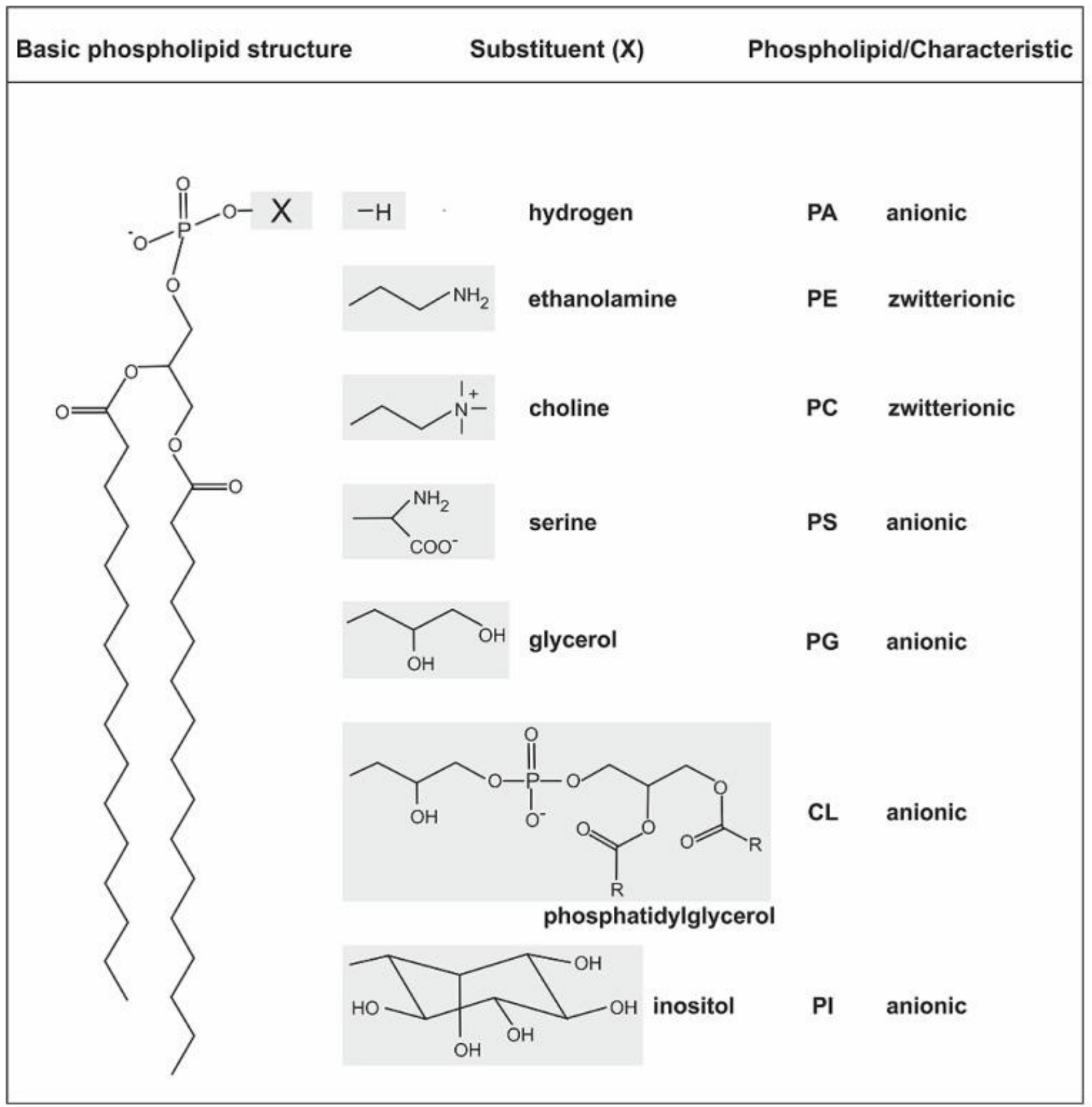

Figure 1-5. Structures of phospholipid headgroups. (Source: Adapted from https://www.researchgate.net/figure/General-structure-of-phospholipids-andcommon-head-groups-PLs-contain-two-fatty-acids_fig1_261605192) 
with proteins. ${ }^{35}$ The most abundant protein found in the VH is albumin. ${ }^{36}$ Ascorbic acid amounts are found to be lower in VHs from diabetics relative to non-diabetics. ${ }^{37}$ Glucose levels of entire VHs are about 2-3 times higher in diabetic than non-diabetic ones obtained from vitrectomies using blood glucose meters. ${ }^{38,39}$ Regional values of PLs and glucose have yet to be reported in the $\mathrm{VH}$ and will be further investigated.

\section{DEVELOPMENT OF THE VH}

There are three main stages, primary, secondary, and tertiary during the development of the vitreous. The stages occur during fetal development. The primary vitreous begins to form around 3-4 weeks after gestation from the surface ectoderm. ${ }^{40}$ Optic vesicles touch the surface ectoderm and secrete mesodermal cells into the vitreous space. ${ }^{41}$ The mesodermal cells turn into the hyaloid artery creating a vasculature network throughout. ${ }^{41}$ Lastly, the choroidal fissure closes, which makes the eye a closed system and intraocular pressure is established. ${ }^{40}$ The secondary vitreous then begins to develop around 6-12 weeks from secretions of the neuroectodermal particles from the retina. ${ }^{41,42}$ It is composed mainly of type II collagen creating an extracellular matrix. The secondary vitreous compresses the primary vitreous and the space between the two becomes Cloquet's canal ${ }^{41}$. During the development of the secondary vitreous, the hyaloid vasculature in the primary vitreous degenerates starting anteriorly towards the posterior region until blood flow ceases after 4 months. ${ }^{41,42}$ Phagocytes break down the vessel walls and hyalocytes and fibroblasts are deposited on the vitreous cortex and base that later synthesize hyaluronan, collagen, and glycoproteins. ${ }^{40,41}$ The final stage of development is the tertiary vitreous, which is notpart of the VH. It is developed from 
secondary vitreous fibrils that condense and elongate to form the lens zonules. ${ }^{40}$ The stages of vitreal development can be seen in figure 1-6.

\section{VH FUNCTIONS}

Several functions of the VH have been reported. One of the vitreal functions is allowing light to reach the retina without being scattered. This is possible due to the transparency of the $\mathrm{VH}$, which contains very low levels of macromolecules, preventing light from being scattered. ${ }^{20}$ Having a refractive index of 1.33 , which is the same as that of water, allows light to be further focused between the lens and retina. Another function of the $\mathrm{VH}$ includes providing support during ocular growthand helping to maintain spherical shape of the eye. ${ }^{20,17}$ The $\mathrm{VH}$ is also important in protecting the lens from oxidation and other ROS migrating towards the lens from the retina. The VH also protects the retina from UV radiation with antioxidants, such as ascorbate. ${ }^{43,44}$ It is possible that the regional changes in the HA-collagen network may serve other functions yet to be elucidated. The $\mathrm{VH}$ serves as a repository for the surrounding ocular regions. The role that these "waste" compounds play in liquefaction is still unknown.

\section{AGEING AND LIQUEFACTION OF THE VH}

The VH undergoes liquefaction as it ages. This process has been reported as early as age 4 in humans and $50 \%$ of the $\mathrm{VH}$ becomes liquefied by the time a person is in his/her 80s (Fig.1-7). ${ }^{20}$ There are many hypotheses on how or why the VH liquefies: enzymatic activity disrupts the collagen network; type IX collagen breaks down causing 


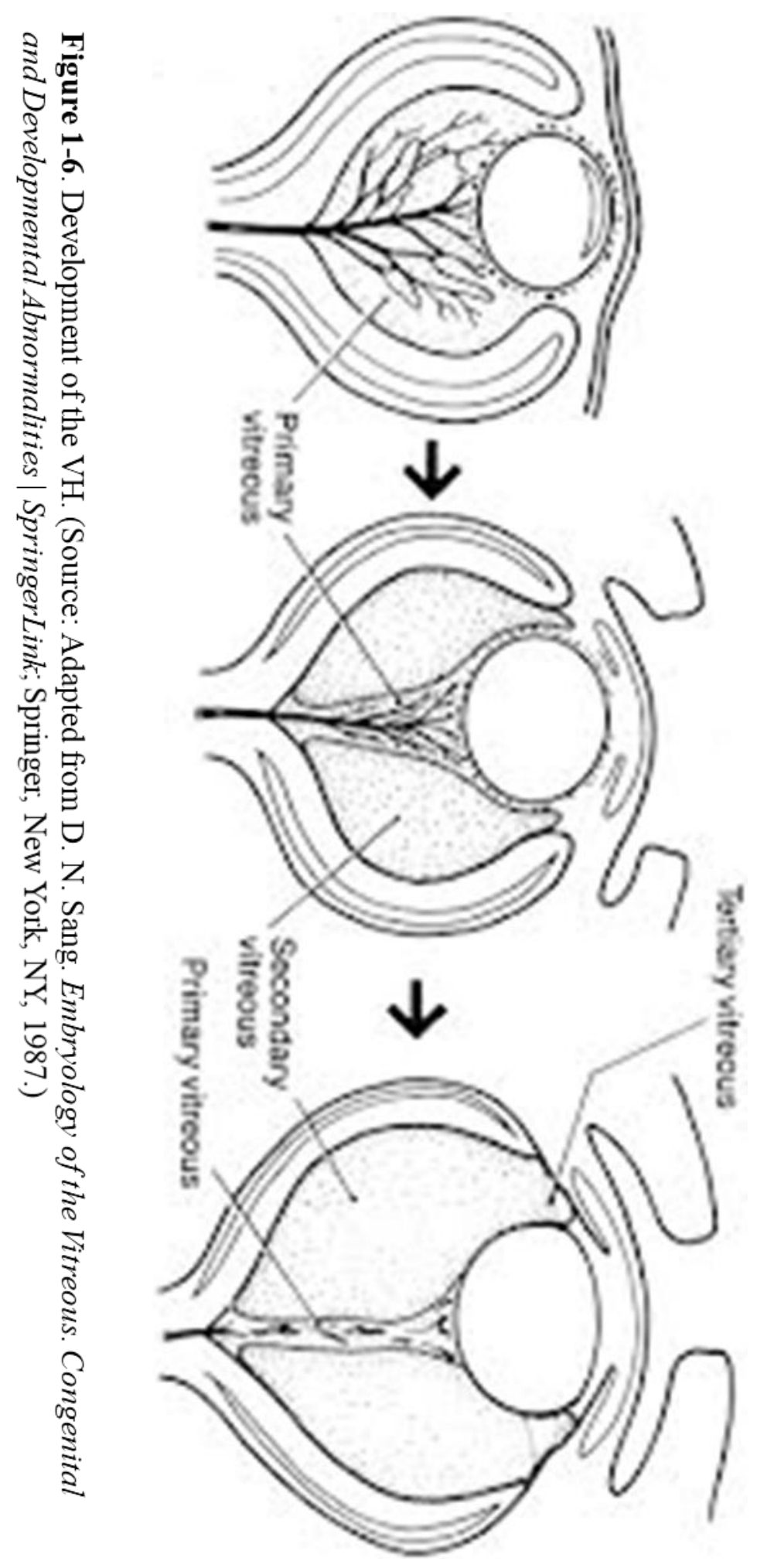




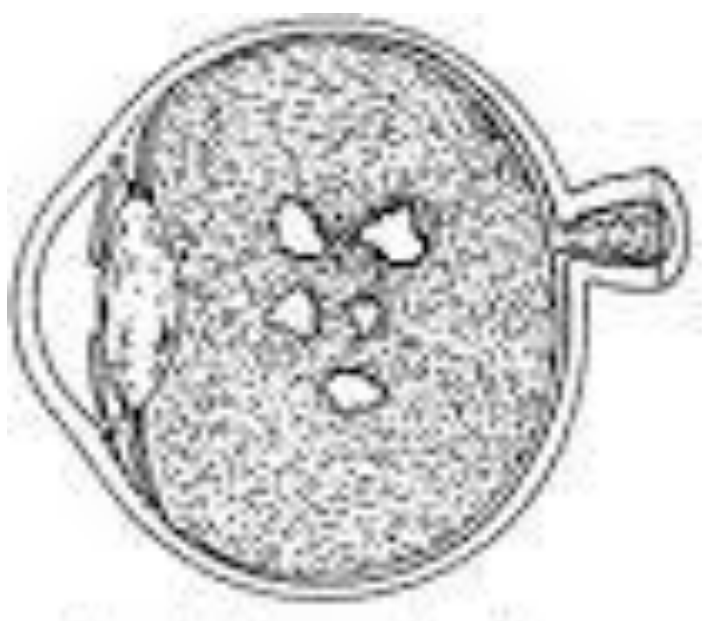

Early liquefaction

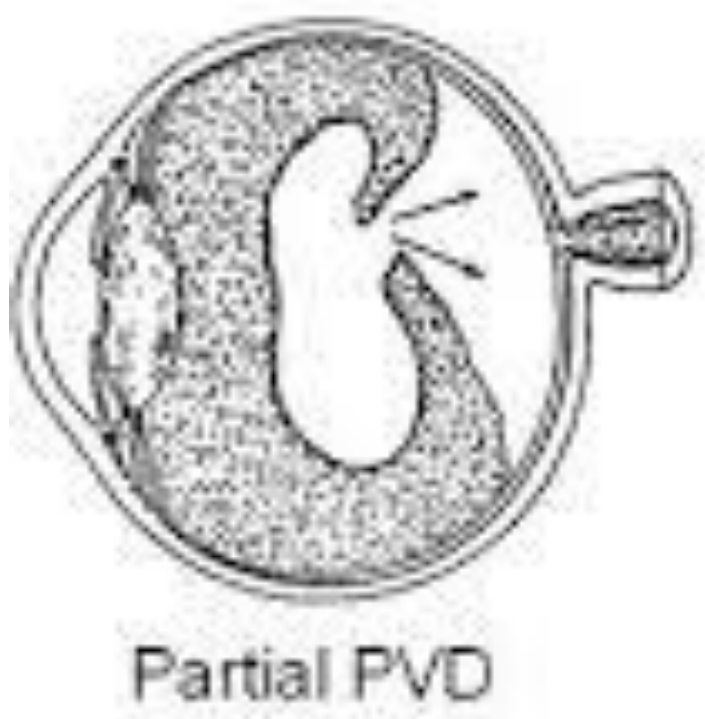

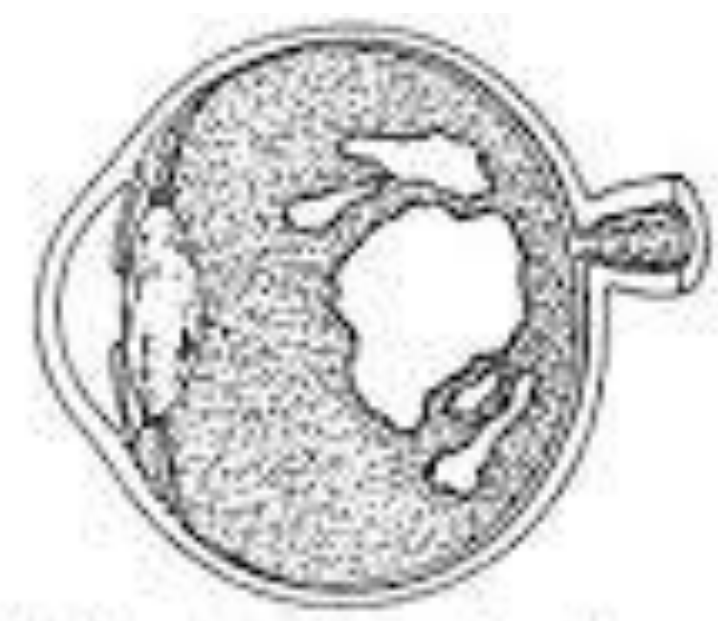

Extensive liquefaction

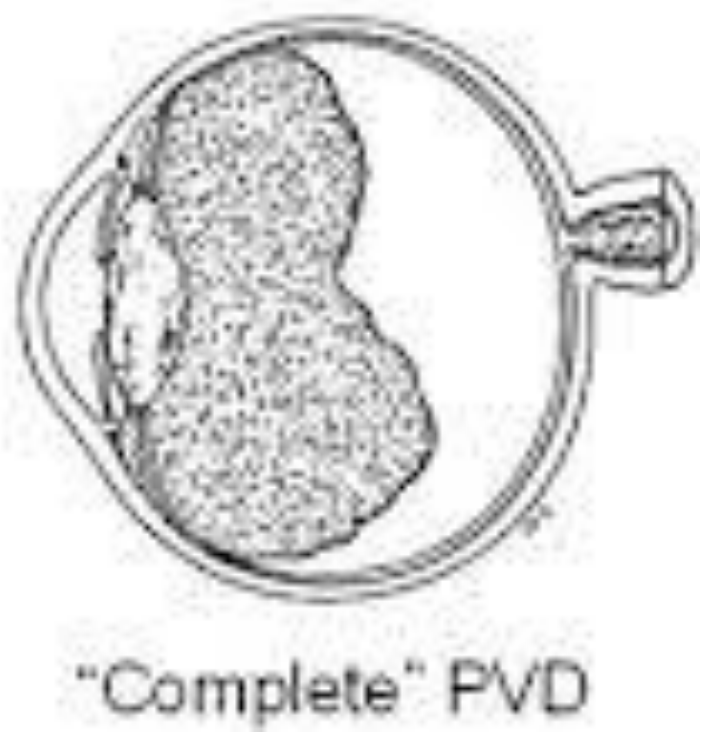

Figure 1-7. Liquefaction of the VH. (Source: Adapted from Le Goff, M. M.; Bishop, P. N. Adult Vitreous Structure and Postnatal Changes. Eye 2008, 22 (10), 1214-1222.) 
type II collagen to stick to itself; HA breaks down; collagen and HA interactions are hindered due to a structural change in collagen from oxidation. ${ }^{20,23}$

When HA was degraded in the VH by injecting hyaluronidase, the VH gel shrunk but did not liquefy. ${ }^{20}$ After a day, the VH turned cloudy but eventually cleared up after a month indicating that HA is always being synthesized in some capacity. ${ }^{45}$ Compared to the addition of hyaluronidase, the VH was more liquefied when lysine-glucose and amino acids underwent the Maillard Reaction. ${ }^{46}$ The Maillard products promoted liquefaction in the VH suggesting glucose could play a role in the increased liquefaction rate of diabetic VHs. Collagenase was also added to the VH to fragment the collagen and the VH did undergo liquefaction. ${ }^{20}$ When transmission electron microscopy was used to look at a liquefied $\mathrm{VH}$, breakdown in collagen fibrils was observed in the liquefied regions compared to the gel regions of the $\mathrm{VH}^{47}$ The fibrils of younger $\mathrm{VHs}$ are in tight networks but are not fused together in any way and collagen type IX is used to space out the network. As the VH gets older the fibril network aggregates due to the collapse of the spacers and liquefaction occurs. ${ }^{20,48}$ Fluorescein was found to disperse more easily in a liquefied $\mathrm{VH}$ than a gelled one, which indicates there is more flow once the $\mathrm{VH}$ is liquefied and this can contribute to ocular diseases. ${ }^{49}$

Liquefaction is a serious problem because it can lead to harmful eye diseases including posterior $\mathrm{VH}$ detachment, macular hole formation, and retinal detachment. ${ }^{48}$ In diabetic patients, the liquefaction process occurs earlier in life and can lead to more serious diseases such as diabetic retinopathy.

\section{Diseases associated with liquefaction}


Diseases associated with vitreal liquefaction include cataracts, posterior vitreal detachment, macular hole formation, retinal detachment, and in patients with diabetes, diabetic retinopathy. Cataracts can result from oxidation of the lens. In gelled VHs, oxygen from the retina is consumed by the VH before it reaches the lens. However, when the $\mathrm{VH}$ is liquefied it no longer consumes the oxygen and therefore the oxygen reaches the lens causing oxidative stress that contributes to cataractogenesis. ${ }^{44}$ The opacity of cataracts cause vision problems.

Posterior vitreal detachment (PVD) is also caused by liquefaction. It takes place when the posterior vitreous separates from the retina due to the adhesion weakening between the two components and affects over 70\% of the population in their 50s. The VH gel shrinks during the liquefaction process causing the $\mathrm{VH}$ to pull away from the retina. With PVD, patients experience flashes of light or "floaters". Floaters consist of collagen fiber aggregates. ${ }^{27}$ They cause blurred vision due to shadows cast on the retina. ${ }^{50}$ Floaters are reported to have various compositions and sizes. ${ }^{51}$ It is possible that part of the hyaloid vessel that breaks down at birth results in fragments that are also part of the floaters. ${ }^{52}$ In adults, floaters are most likely caused by PVD but in adolescence, floaters most likely result from myopic vitreopathy. ${ }^{53}$ Other causes of floater formation include asteroid hyalosis, inflammation, and cataract surgery. A proposed treatment for floaters is focusing a laser onto the floater to disrupt the collagen strands of floaters. ${ }^{51} \mathrm{~A}$ treatment proven effective is performing a vitrectomy. ${ }^{50}$

Macular hole formation results from the $\mathrm{VH}$ pulling away from the retina and causing the macula to tear. ${ }^{54}$ Symptoms include distorted and blurred central vision. Retinal detachment occurs when liquid from a liquefied vitreous enters a tear in the retina 
causing the retina to pull away from the retinal pigment epithelium layer. ${ }^{55}$ Patients experience floaters and flashes in their vision and eventually the peripheral vision goes dark.

\section{BARRIERS AROUND THE VH}

Electrolytes enter the $\mathrm{VH}$ through the ciliary body and the aqueous humor but non-electrolytes enter from the choroidal and retinal areas. The ciliary body, choroidal, retinal, and optic nerve tissues provide a blood VH barrier. Therefore, the passage of molecules into the $\mathrm{VH}$ depends on barriers. ${ }^{56}$ There are two main barriers in the eye, the blood-aqueous barrier (BAB) and the blood-retinal barrier (BRB). The BAB consists of non-pigmented epithelium of the ciliary body, the posterior iris epithelium, and the iris vessel and Schlemm's canal endothelium. ${ }^{57}$ It is responsible for providing nutrition to the lens and cornea. The ciliary body regulates the $\mathrm{BAB} .{ }^{58}$ There is a complex interface between the retinal basal lamina, which is composed of type IV collagen and glycoproteins, and the vitreous cortex known as the BRB. ${ }^{59}$ There is an active transport from the $\mathrm{VH}$ to the retinal vessels and a passive transport from the $\mathrm{BRB}$ into the $\mathrm{VH} .^{57}$ An increase of glucose transport from retinal vessels into the $\mathrm{VH}$ occurs in patients with diabetes. ${ }^{58}$ A high passive transport level can be correlated to the degree of retinopathy, which is a direct effect of the breakdown of the BRB. ${ }^{60}$

\section{EFFFECT OF DIABETES ON OCULAR TISSUE}

Diabetes causes hyperglycemia that leads to damage of the vasculature system. An increase in oxidative stress is seen with this disease and causes the formation of free 
radicals, which leads to glucose oxidation, glycation of proteins, and a decrease in antioxidants. ${ }^{61,62}$ Some of the oxidative stress biomarkers are superoxide dismutase, catalase, glutathoine, and lipid peroxidation. ${ }^{61}$ This leads to many ocular diseases such as diabetic retinopathy, which involves the degeneration of blood vessels in the retina most likely from inflammation and other factors. ${ }^{63}$ Higher levels of glucose (Non-Diabetic (ND): 0.7, Diabetic (D): 3mM), sorbitol (ND:0.3, D:1.7mM), and fructose (ND: 0.4, D: $1.2 \mathrm{mM}$ ) are found in lenses of diabetics relative to those from non-diabetics. ${ }^{64}$

Diabetics have higher levels of glucose and lactate in the $\mathrm{VH}$, which can cause serious problems. ${ }^{65}$ The transport of glucose into the $\mathrm{VH}$ is of interest due to the higher serum levels reported in diabetics. Glucose is transported into the retina by facilitated diffusion through GLUT1 and GLUT3 transport proteins. ${ }^{42}$ The transport of glucose in the VH was studied using fluorescence. Fluorescein was injected into the blood and the transport between the retina and $\mathrm{VH}$ was studied. Fluorescein favored the movement of the $\mathrm{VH}$ to the retina more than the retina to the $\mathrm{VH}$. However, a longer time for the dye to move out of the VH was noted in diabetic tissue due to the increased flow of dye into the $\mathrm{VH} .{ }^{66}$ The transport of molecules into the $\mathrm{VH}$ increases with exposure to diabetes because of higher permeability most likely a result from changes to the epithelial barrier,

changes to the inner retinal vessels, and the effect of glycosylated proteins on transport. ${ }^{67}$ Absorption in the UV-Vis and near-infrared, polarimetry, thermal emission, Raman spectroscopy, fluorescence, and ultrasound have been used to detect glucose in serum. ${ }^{68}$

\section{AGEs, ROS, etc.}

Advanced glycation end products (AGEs), which are elevated in diabetics, can be used to measure the glycemic control and measure protein oxidation for amounts of reactive 
oxygenated species (ROS) caused by hyperglycemia. ${ }^{69}$ ROS can cause lipid oxidation of polyunsaturated lipids by converting $\mathrm{H}_{2}$ to $\mathrm{HOOH}$ or can cause lipid peroxidation. ${ }^{70}$ Changes in the $\mathrm{m} / \mathrm{z}$ value could indicate a free radical attack on lipids. ${ }^{71}$ Common products of lipid oxidation are lipids with shortened chains containing hydroxyl, carbonyl, or carboxyl groups. ${ }^{72}$ Another consequence of diabetes is protein glycation. Glycation occurs on the lysine, arginine, or N-terminal of proteins and involves nonenzymatic covalent attachment of a reducing sugar. ${ }^{73}$ For example, the carbonyl group of glucose interacts with the amino group on lysine and forms a Schiff base that undergoes an Amadori rearrangement to fructosamine and can be detected using mass spectrometry and looking for a M+162 $\mathrm{Da}$ of the modified protein. ${ }^{74}$ Protein-bound fluorescence can measure products of AGE products. ${ }^{75}$ AGEs increase the formation of ROS that reduce pericytes and cause the breakdown of the BRB, prevent antioxidants from working properly, activate factor that increase inflammatory cytokines, and alter protein structure and functions. ${ }^{76}$ AGEs can be created from oxidation and glycation and produce pentosidine and $\mathrm{N}$-carboxymethyl lysine. The main target of sugars is collagen, which forms methylglyoxal-modified collagen.

\section{ANALYTICAL TECHNIQUES USED TO STUDY THE VH}

\section{Spectroscopy techniques}

Spectroscopic techniques that have been used to study the $\mathrm{VH}$ are nuclear magnetic resonance (NMR) and Raman spectroscopy. ${ }^{77}$ Raman Spectroscopy has mainly been used to study the lens so far. Differences in the lens with and without diabetes have been reported. NMR has also been used to study the VH. The Carr Purcell method was 
used to measure $T_{1}$ and $T_{2}$. Shorter relaxation times of both $T_{1}$ and $T_{2}$ were noted with lower viscosity. ${ }^{78}$ The relaxation times were also shorter with the addition of collagenase to the VH. The addition of hyaluronidase had minor changes and ascorbic acid had no observable change in the relaxation times.

\section{In-vivo techniques}

In-vivo techniques such as dynamic light scattering, and optical coherence tomography have previously been used to study the $\mathrm{VH} .{ }^{77}$ Dynamic light scattering has been used to observe changes in the structure from diabetic vitreopathy. Optical coherence tomography has been used to measure the size and distance between structures in the VH.

\section{In-vitro techniques}

The VH has also been studied using dark-field slit microscopy as an in-vitro technique, which showed the structure of collagen and age related changes on the overall vitreous structure. ${ }^{77}$ Even with all of these techniques, there has not been a noninvasive technique reported to image the $\mathrm{VH}^{79}$

\section{Techniques used to study distribution of PLs}

Since the VH is such a dilute system, molecular concentrations are extremely low requiring very sensitive techniques to detect PLs and other constituents of the VH. In the past, PLs have been quantified in ocular tissues (lens and retina) using ${ }^{31} \mathrm{P}$ NMR. ${ }^{80,81,82,83}$ Unfortunately, this approach does not provide the sensitivity needed to establish the regional PL composition of VHs. So far, matrix-assisted laser desorption ionization (MALDI) mass spectrometry (MS) has been successfully applied to study the distribution

of lipids, proteins, and other metabolites throughout the retina and lens. ${ }^{84,85,86,87,88,89,90}$ 
This report focuses on the development of analytical methodologies to determine the source and to quantify the ex-situ and in-vitro regional distribution of PLs in porcine VHs. Determining compositional differences in various regions of the VH will help us explore compositional changes that may contribute to the liquefaction of VHs.

The two analytical methods used to explore the $\mathrm{VH}$ are matrix-assisted laser desorption ionization mass spectroscopy (MALDI/MS) and nuclear magnetic resonance spectroscopy (NMR). The next sections address the principles and applications of MS and NMR.

\section{MASS SPECTROSCOPY}

\section{Mass spectroscopy background}

According to Webster's dictionary, mass spectroscopy (MS) is "an instrumental method for identifying the chemical constitution of a substance by means of the separation of gaseous ions according to their differing mass and charge". MS involves the ionization of analytes and analysis of their mass-to-charge ratios $(\mathrm{m} / \mathrm{z}) .{ }^{91}$ The ion sources can either involve hard ionization or soft ionization. Hard ionization promotes the molecules to an excited state and then the relaxation process involves the breaking of bonds, which causes fragmentation of the parent ion. Soft ionization causes little, if any, fragmentation of the parent ion. As a result, the most intense peak occurs at the $\mathrm{m} / \mathrm{z}$ value of the parent ion. Some of the most commonly used ionization sources include electron ionization (EI), chemical ionization (CI), electrospray ionization (ESI), matrix-assisted laser desorption ionization (MALDI), fast atom bombardment (FAB), and secondary ion mass spectrometry (SIMS), and desorption electrospray ionization (DESI). ${ }^{92}$ 


\section{Types of ionization sources}

Electron ionization:

In EI, the analyte in the gas phase is ionized using a beam of energetic electrons (70eV) from a heated filament source, which is usually $\mathrm{W}$ or $\mathrm{Re} .{ }^{93}$ Upon impact, radicals are produced and their instability leads to fragmentation and formation of cations. ${ }^{94} \mathrm{EI}$ is a hard ionization technique so the parent ion fragments to daughter ions. Advantages of EI include high reproducibility, high ionization efficiency, and sensitivity. Disadvantages include only formation of cations, the sample has to be volatile, and limited to low molecular weight (under $600 \mathrm{Da}$ ) compounds. In addition, it cannot be used to analyze mixtures or used for imaging.

Chemical ionization:

$\mathrm{CI}$ involves the use of a reagent gas that at high pressure collides with the analyte to form ions. ${ }^{95}$ The reagent gas can be selected to react with multiple compounds or specific molecules. Less fragmentation occurs using CI compared to EI, which makes it a softer form of ionization but it is still not applicable for imaging. ${ }^{96}$

Electrospray ionization:

In ESI, the analytical solution, in a polar solvent, travels through a capillary tube with a conductive tip held at a high voltage at a low flow rate. A positively or negatively charged spray is produced..$^{97}$ If positive, the anions move to the tip of the capillary tube and the cations toward the inlet of the MS. It passes through a desolvating tube where the solvent is evaporated and the analyte forms charged droplets. ${ }^{98}$ The droplets become smaller and smaller as the solvent evaporates causing an increase in the charge density until the Rayleigh limit is reached and the repulsion increases and overcomes surface 
tension. Coulombic explosions occur and as the solvent evaporates multiply charged ions are produced ${ }^{93}$ ESI is a soft ionization method and can be used on low or high molecular weight molecules but can not be used for imaging. For ESI, the salt content of the analyte needs to be extremely low to prevent clogging of the capillary tube.

Fast atom bombardment:

FAB requires the use of a matrix, often an organic compound with low vapor pressure such as glycerol. ${ }^{99} \mathrm{~A}$ fast particle beam focuses on the analyte and matrix mixture. The most common particle beams are Ar or Xe at $4-10 \mathrm{keV} .{ }^{100}$ The kinetic energy from the beam is transferred to the analyte mixture and collisions cause desorption and ionization of the analyte. ${ }^{100}$ FAB has high ionization efficiency, low detection limits, and can be used for generation of positive or negative ions. Even though it is known as a soft ionization technique, fragments of the parent ion are produced and matrix-related ions complicate the spectrum. This ionization method is not applicable for imaging.

Secondary ion:

In SIMS, an ion beam, usually $\mathrm{Ar}^{+}$or $\mathrm{Cs}^{+}$, is directed to the surface of the solid analyte causing 'sputtering' of secondary ions. ${ }^{101}$ The parent ion is not seen and is fragmented. The power of the ion beam is responsible for the ionization efficiency and the amount of fragmentation. Imaging is possible in SIMS due to the ability to focus the ion beam, which leads to good special resolution. ${ }^{102}$ However, since only fragments are detected, the parent ion(s) from which they derive cannot be determined. SIMS has been used for imaging applications.

Desorption electrospray ionization: 
DESI operates at atmospheric pressure. An electrically charged mist is focused into the surface of the analyte and is attracted to the surface by applying a voltage on the sample. ${ }^{103}$ Ionization of the analyte results from the formation of charged analyte-solvent droplets. The ions are desorbed and carried to the MS analyzer. ${ }^{93}$ Limitations of DESI include poor spatial resolution and potential sample degradation upon solvent interaction. However, it still can be used for imaging.

The ionization technique used for this project is MALDI and will be explored in more detail in the following sections.

\section{Mass Analyzers}

Common types of mass analyzers used in MS are magnetic sector analyzer (measures momentum, resolution: $10^{5}$ ), quadrupole ion trap (measures frequency, resolution: $10^{4}$ ), cyclotron resonance (ICR) analyzer (measures frequency, resolution: $10^{6}$ ), time of flight (TOF) detector (measures flight time, resolution: $10^{3}$ ).

Magnetic sector:

A magnetic sector analyzer works by accelerating the ions into a flight tube. The ions are separated based on their charge and velocity. The ions travel in an arc due to the applied magnetic field. The size of the arc depends on the $\mathrm{m} / \mathrm{z}$ value. The larger values will have a larger arc. The magnetic field is scanned over a variety of strengths. Specific magnetic field strengths will allow only specific $\mathrm{m} / \mathrm{z}$ values to be detected. If the ion does not have the selected $\mathrm{m} / \mathrm{z}$ value, it will have a trajectory that will not allow it to pass through to the detector. The $\mathrm{m} / \mathrm{z}$ value detected is based on the magnetic field strength, $\mathrm{B}$, the accelerating voltage, $\mathrm{V}$, and the radius of the circular path, $\mathrm{r}$, from the following equation: $\mathrm{m} / \mathrm{z}=\mathrm{B}^{2} \mathrm{er}^{2} / 2 \mathrm{~V} .{ }^{104}$ 
Quadrupole:

A quadrupole mass analyzer consists of four parallel rods that have DC voltages of opposite signed applied to opposite rods. Two of the rods are positively charged and two are negatively charged. Ions drift through the four parallel rods. Variable AC potentials are applied and cause the ions in the detector to oscillate around the center of the rods. When the voltage is changed, the $\mathrm{m} / \mathrm{z}$ value of the resonant ion can be controlled and causes the non-resonant ions to collide with the rods and filter out. ${ }^{105} \mathrm{The} \mathrm{m} / \mathrm{z}$ value detected depends on the frequency of the rf field, $\mathrm{f}$, and the internal radius of the quadrupole, $\mathrm{r}_{0}$, in the following equation: ${ }^{93}$

$$
\mathrm{m} / \mathrm{z}=0.069 \mathrm{~V} / \mathrm{f}^{2} \mathrm{r}_{\mathrm{o}}{ }^{2}
$$

Another type of quadrupole is an ion trap, which is a 3D version of the quadrupole and has 3D electric fields to store ions. For a quadrupole ion trap, a radiofrequency is applied to the ring electrode and the two exit endcap electrodes are grounded. Ions with the targeted $\mathrm{m} / \mathrm{z}$ value circulate in the stable orbit of the cavity surrounded by the ring. When the voltage of the trap is increased, the orbits of the heaver ions are stabilized and the lighters ones are destabilized and collide with the ring electrode. The equation to determine the $\mathrm{m} / \mathrm{z}$ for the ion trap is $:^{93}$

$$
\mathrm{m} / \mathrm{z}=0.1075 \mathrm{~V} / \mathrm{f}^{2} \mathrm{r}_{\mathrm{o}}^{2}
$$

\section{Cyclotron resonance}

In ICR, the ions enter the detector and are trapped in a circular orbit that is perpendicular to the applied magnetic field. ${ }^{106}$ Their radius depends on the ion velocity. The trapped ions are excited from the applied oscillating electric field perpendicular to the magnetic field. The ion can absorb energy from an applied electric field if the 
frequency of the field matches the cyclotron frequency. The absorbed energy increases the velocity of the ion and the orbital radius increases. Once the electric field is removed the radius remains increased. To determine the $\mathrm{m} / \mathrm{z}$ value detected, the fixed magnetic field, $\mathrm{B}$, and the cyclotron frequency, $\mathrm{f}_{\mathrm{c}}$, must be known in the following equation: ${ }^{93}$

$$
\mathrm{m} / \mathrm{z}=\mathrm{B} / \mathrm{f}_{\mathrm{c}}
$$

The TOF analyzer is commonly used with MALDI and will be further explored in the following section.

\section{MALDI}

\section{Basic principles of MALDI}

Franz Hillenkamp and Michael Karas developed MALDI in 1985. MALDI is a soft form of ionization and requires the use of a matrix. The matrix needs to be significantly more concentrated than the analyte, co-crystallize with the analyte, and be able to absorb at the wavelength of the laser. ${ }^{104,107}$ The matrix and analyte can be "premixed" before spotting small aliquots on a stainless steel MALDI or applied using the "dried droplet" method where the analyte is spotted on the plate, dried, and the matrix is spotted on top of the spot. The co-crystalized complex is dried and inserted into a chamber, which is usually at a high vacuum pressure $\left(10^{-7}\right.$ Torr). A laser is fired directly on the samples and the matrix absorbs the radiation. ${ }^{107,108}$ The energy causes the matrix to be promoted to a higher electronic state and transfer part of the energy to the analyte. The matrix and analyte are desorbed and form a plume of ions that travel to the mass analyzer where they are separated by their mass-to-charge ratios (m/z) (fig. 1-8). ${ }^{109}$ MALDI allows for a wide range of molecular weights to be generated with minimal fragmentation. 


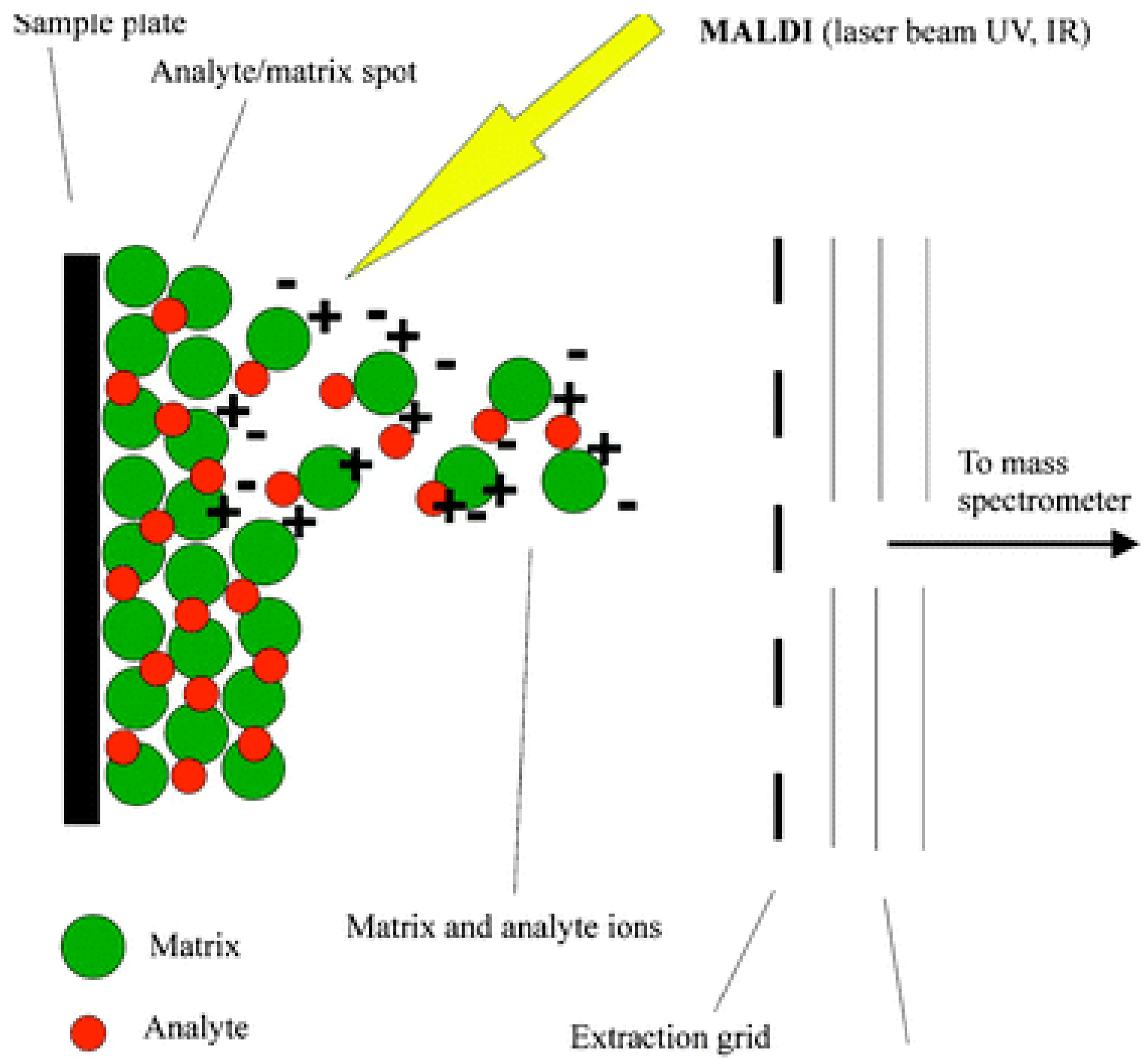

Focussing lens

Figure 1-8. A laser is fired at the co-crystallized matrix-analyte mixture on the MALDI plate. The ions are directed into the mass analyzer. (Source: Adapted from Petković, M.; Kamčeva, T. FAB, ESI and MALDI Mass Spectrometric Methods in the Study of Metallo-Drugs and Their Biomolecular Interactions. Metallomics 2011, 3 (6), 550-565.) 
However, MALDI has low spatial resolution particularly if inhomogeneous crystallization takes place.

\section{Mechanisms for ionization in MALDI}

Ionization occurs from a combination of primary and secondary ionization reactions. ${ }^{110}$ Primary ionization reactions occur from the laser pulse and immediately produce ions. ${ }^{11}$ Secondary ionization reactions occur in the ion plume after laser ablation from the primary ions interacting with neutral species. ${ }^{111}$

Multiple mechanisms of primary ionization reactions have been reported. Some examples are multiphoton ionization, exciton pooling, excited-state proton transfer, and desorption of preformed ions. ${ }^{112,113}$ Multiphoton ionization involves the matrix molecules absorbing two or three photons from laser radiation and forming a radical. ${ }^{112}$ This mechanism is not favored due to the ionization potentials of the matrices, which would require the addition of multiple photons to form a radical. Exciton pooling involves multiple excited matrix molecules being in close proximity to each other. The molecules

redistribute their energy to one molecule, which is now able to form a radical. ${ }^{107,113,114}$ Excited-state proton transfer occurs when a matrix molecule is in an excited state and accepts a proton before relaxation. ${ }^{112}$ The matrix can accept a proton more readily due to the donor is more acidic, which favors donating a proton to the matrix. The simplest primary mechanism is desorption of preformed ions. Ions are present in the solid state and upon laser firing, the ions are desorbed from the sample. ${ }^{112}$

Secondary mechanisms are reactions occurring in the ion plume based on thermodynamics and kinetics. ${ }^{110,113}$ They include proton transfer, cation transfer, electron transfer, and electron capture which are thought to occur in the gas phase. ${ }^{112}$ Proton 
transfer involves the transfer of a proton from the matrix to the analyte. The analyte has a higher affinity for protons than the matrix favoring the reaction. If the analyte has a low affinity for a proton then the analyte is detected in its deprotonated form.

$$
\begin{gathered}
{[\text { matrix }+\mathrm{H}]^{+}+\text {analyte } \leftrightarrow \text { matrix }+[\text { analyte }+\mathrm{H}]^{+}} \\
{[\text {matrix }-\mathrm{H}]^{-}+\text {analyte } \leftrightarrow \text { matrix }+[\text { analyte }-\mathrm{H}]^{-}}
\end{gathered}
$$

Cation transfer occurs when a cation is transferred to the analyte.

$$
[\text { matrix }+\mathrm{Na}]^{+}+\text {analyte } \leftrightarrow \text { matrix }+[\text { analyte }+\mathrm{Na}]^{+}
$$

Electron transfer involves transferring an electron from the analyte to the more favorable cations from the matrix.

$$
[\text { matrix }]^{+}+\text {analyte } \leftrightarrow \text { matrix }+[\text { analyte }]^{+} \text {or }[\text { matrix }]^{-}+\text {analyte } \leftrightarrow \text { matrix }+[\text { analyte }]^{-}
$$

For the electron capture reaction, excess electrons are needed and captured by the analyte using the negative mode. The intensity of ions is affected by the homogeneity of the matrix-analyte mixture, amount of collisions occurring in the ion plume, and the temperatures reached in the plume to achieve activation energies. ${ }^{115}$

\section{Previous matrices used for MALDI}

The matrix choice is of utmost importance for MALDI/MS. A good matrix produces a high signal-to-noise ratio for analyte peaks, absorbs at the laser emission wavelength, produces minimal matrix related peaks to avoid interference with analyte peaks, and is not likely to form cluster ions. ${ }^{116}$ The type of analyte will factor in to which matrix will work the best for analysis. For example, a common matrix for proteins and peptides is 4hydroxy- $\alpha$-cyanocinnamic acid. ${ }^{117}$ However, this matrix gives poor results for the detection of lipids. For our studies, we focus on the analysis of lipids and sugars. This section will focus on previously used matrices for these molecules. 
Commonly used matrices for lipids include 2,5-dihydroxybenzoic acid (DHB), graphite, para-nitroaniline (PNA), 9-aminoacridine (9-AA), and 2-(2-Aminoethylamino)5-nitropyridine (AAN). ${ }^{118-120}$ DHB is one of the most popular choices for the analysis of lipids in the positive mode. AAN and 9-AA have shown promising results in the negative mode. ${ }^{121}$ Good results have also been reported using PNA in both the positive and negative modes. ${ }^{85,122}$ However PNA presents a serious limitation: it sublimes under vacuum conditions. ${ }^{123,124}$ Short acquisition times are needed thus limiting its application in lengthy imaging experiments. ${ }^{125}$

Sugars are harder to ionize than lipids. To our knowledge there is not a standard matrix used for detecting sugars. Different matrices have been used successfully to ionize sugars. Some of the matrices include charcoal, 3-aminoquinoline, N-(1-naphthyl) ethylendiamine dihydrochloride, carbon nanotubes, ionic liquid, and DHB. ${ }^{126-129}$ Our group has had the most success using DHB to detect glucose.

\section{Basic principles of TOF analyzer}

The TOF detector allows ions to be separated based on their $\mathrm{m} / \mathrm{z}$ values (fig. 1-9). The TOF analyzer is also compatible with extracting both positive and negative ions by adjusting the polarity of the main voltage source. ${ }^{130}$ The analyte is ionized and the ions are accelerated into the analyzer from an applied voltage by an electric field. The applied voltage is usually set between $15,000-25,000 \mathrm{~V}$ depending on the $\mathrm{m} / \mathrm{z}$ of the ions of interest. All ions theoretically enter the drift tube with the same kinetic energy (KE) and are related to the mass of the ion, $\mathrm{m}$, and the velocity travelled, v. KE is also related to the applied voltage, $\mathrm{V}$, electron charge, e, and the charges of the ion, $\mathrm{z}^{93,131}$

$$
\mathrm{KE}=1 / 2 \mathrm{mv}^{2}=\mathrm{zeV}
$$




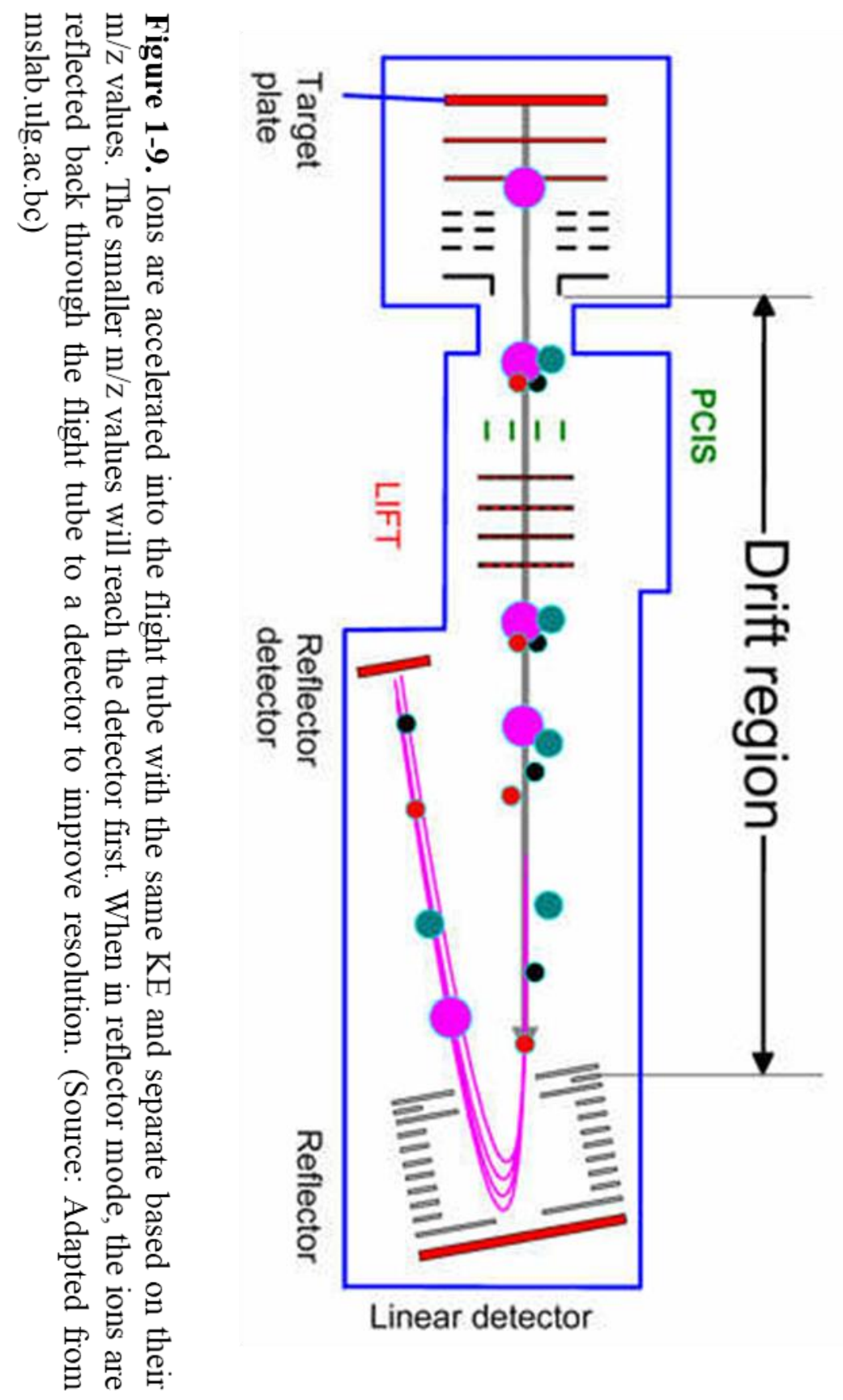


The velocity the ion travels depends on its mass. The drift tube has no electromagnetic fields so the ions can travel at constant velocities for a set drift length, D. The velocity is related to $\mathrm{D}$ and the time travelled, $\mathrm{t}$.

$\mathrm{V}=\mathrm{D} / \mathrm{t}$ Therefore, the smaller mass ions will travel faster and reach the detector before the larger mass ions. The $\mathrm{m} / \mathrm{z}$ of the ion can be calculated using the length of the drift tube, $\mathrm{D}$, the time of flight, $\mathrm{t}$, and the $\mathrm{KE}$ in the following equation: ${ }^{93}$

$$
\mathrm{m} / \mathrm{z}=2 \mathrm{t}^{2} \mathrm{eV} / \mathrm{D}^{2}
$$

TOF analyzers have poor mass resolution because of the varying times of ionization of ions. To overcome this limitation, delayed extraction is utilized. A delayed extraction can improve mass resolution by allowing ions with the same $\mathrm{m} / \mathrm{z}$ to reach the detector at the same time. ${ }^{132}$ A delayed extraction involves a delay in between the laser pulse and the application of the accelerating voltage. This theoretically allows all of the ions to be accelerated into the drift tube at the same time.

A TOF detector can operate in a linear mode or reflector mode. In the linear mode, the ions travel the length of the drift tube to reach the detector. Since the reflector mode gives significantly better resolution, linear mode is usually only used when the ions are too unstable to travel back through the drift tube. The reflector mode involves the ions traveling the length of the tube and then they are reflected back through the tube from a relectron to a detector. A reflectron is made up of a series of rings at the end of the drift tube that have applied voltages. The voltage of the rings increases linearly. Ions penetrate the reflectron until they no longer have any KE. Then they are accelerated in the opposite direction into the drift tube with their initial KE. The ions that have a higher KE, 
penetrate deeper into the electric rings than the ones with less KE. The reflectron corrects for varying arrival times to the detector and increases the path lengths. ${ }^{93}$

\section{Previous applications of MALDI-TOF}

MALDI has many practical uses. Some of the purposes pertinent to this project are its biological applications and ability to provide qualitative data. Some of the shortcomings seen with MALDI are low reproducibility and difficulty with matrix. The matrix needs to be able to absorb the laser radiation and transfer the energy to the analyte so both can be ionized. The matrix needs to produce homogenous spots to help overcome the lack of reproducibility commonly associated with MALDI. Several matrices are often tried to find one that ionizes a specific analyte. MALDI provides many advantages. It is a soft ionization technique so the analyte usually will not undergo extensive fragmentation, which allows the parent ion to easily be detected. Minimal sample preparation is needed, which allows the sample and matrix to be applied to the plate and analyzed. MALDI is a fast, accurate approach that can be applied for bioanalysis. MALDI has already been used extensively to characterize the regional composition of the lens and retina through imaging experiments. This technique offers the versatility needed to study a delicate tissue such as the vitreous humor and was used in the analysis of the regional composition of the $\mathrm{VH}$.

NMR

\section{Basic principles of NMR spectroscopy}


For an atom to be NMR active, it needs to have a nuclear spin. ${ }^{1} \mathrm{H},{ }^{13} \mathrm{C}$, or ${ }^{31} \mathrm{P}$ have a nuclear spin of $1 / 2$ making them NMR active. Atoms that have a nuclear spin of zero, such as ${ }^{12} \mathrm{C}$, do not give NMR signals. When the nuclei are places in a magnet, they align with the magnetic field and have angular momentum, creating an electrical current. The nuclei spin along their own axis creating a small magnetic field. The frequency the nuclei are spinning is known as the Larmor frequency, $\mathrm{v}_{\mathrm{o}}$, and is linearly related to the magnetic field. ${ }^{133}$ It can be calculated using the strength of the magnetic field, $\mathrm{B}_{0}$, and the magnetogyric ratio, $\mathbf{r}$, which is the strength of the small magnetic field surrounding the nuclei. The magnetogyric ratio varies for different nuclei. For example, ${ }^{1} \mathrm{H}$ has a magnetogyric ratio is four times larger than that of ${ }^{13} \mathrm{C} \cdot{ }^{133}$

$$
v_{0}=\Upsilon B_{0} / 2 \pi
$$

For nuclei with a spin of $1 / 2, \alpha$ and $\beta$ quantum states are possible. In the $\alpha$-state, the nuclei are aligned with the external magnetic field and in the $\beta$-state they oppose the magnetic field. There is a small population difference between the $\alpha$ and $\beta$ states. The energy between the quantum states depends on the strength of the external magnet. The stronger the magnet, the larger the energy gap between states. ${ }^{134}$

$$
\Delta \mathrm{E}=\mathrm{hv}_{\mathrm{o}}
$$

Once the nuclei experience the external magnetic field, a relative small excess of spins are in the alpha state. Upon the application of a short but strong pulse of radiofrequencies, the orientation of the spins flips to the $\beta$-state causing the net magnetization to be in the $\mathrm{z}$ plane (Fig. 1-10). ${ }^{133}$ The nuclei experience a pulse with radiofrequencies applied at $90^{\circ}$ with certain delay times in between pulses. The length of the applied pulse determines the needed tipping angle and changes as $\alpha$-state spins are 
promoted to the $\beta$-state. After the pulse, the excited nuclei return to equilibrium in which an excess $\alpha$-state spins is present. The magnetic field applied to the nuclei induces an energetic transition that is dependent on the electron density around the specific nuclei. ${ }^{93}$ After the pulse, free-induction decay (FID) signals are acquired and the frequency of this decay is related to the chemical shift of a given nucleus. The relaxation causes decay in the signal over time. The FIDs are averaged together and converted to a spectrum after applying the Fourier Transform.

\section{Chemical information obtained from NMR}

Once the nuclei are excited, they relax to their equilibrium state. The relaxation process involves two main types of relaxation, $T_{1}$ and $T_{2} . T_{1}$ is the longitudinal relaxation in the z-plane caused by nuclei magnetic fields oscillating at the Larmor frequency. It is the process of establishing the normal Gaussian population distribution of the $\alpha$ and $\beta$ spin states, and measures the average lifetime of nuclei in the higher energy state by calculating the spins in the beta state returning to the excess $\alpha$ spin state, known as the Boltzman equilibrium. $T_{1}$ is a $1^{\text {st }}$ order exponential decay and is influenced by the mobility of the lattice. The excited spin release energy to the lattice. ${ }^{93}$ When the motions of the molecules in solution are slower, $\mathrm{T}_{1}$ is smaller and more efficient. The most efficient longitudinal relaxation occurs when $T_{1}$ is near the Larmor frequency. Larger molecules or viscous solutions tend to have shorter $\mathrm{T}_{1}$ relaxation times but once the molecular motions are slower than the Larmor frequency, $\mathrm{T}_{1}$ increases (fig.1-11). Quaternary carbons have longer $T_{1}$ values because they have an absence of motional effects compared to carbons bonded to protons. ${ }^{93}$ 


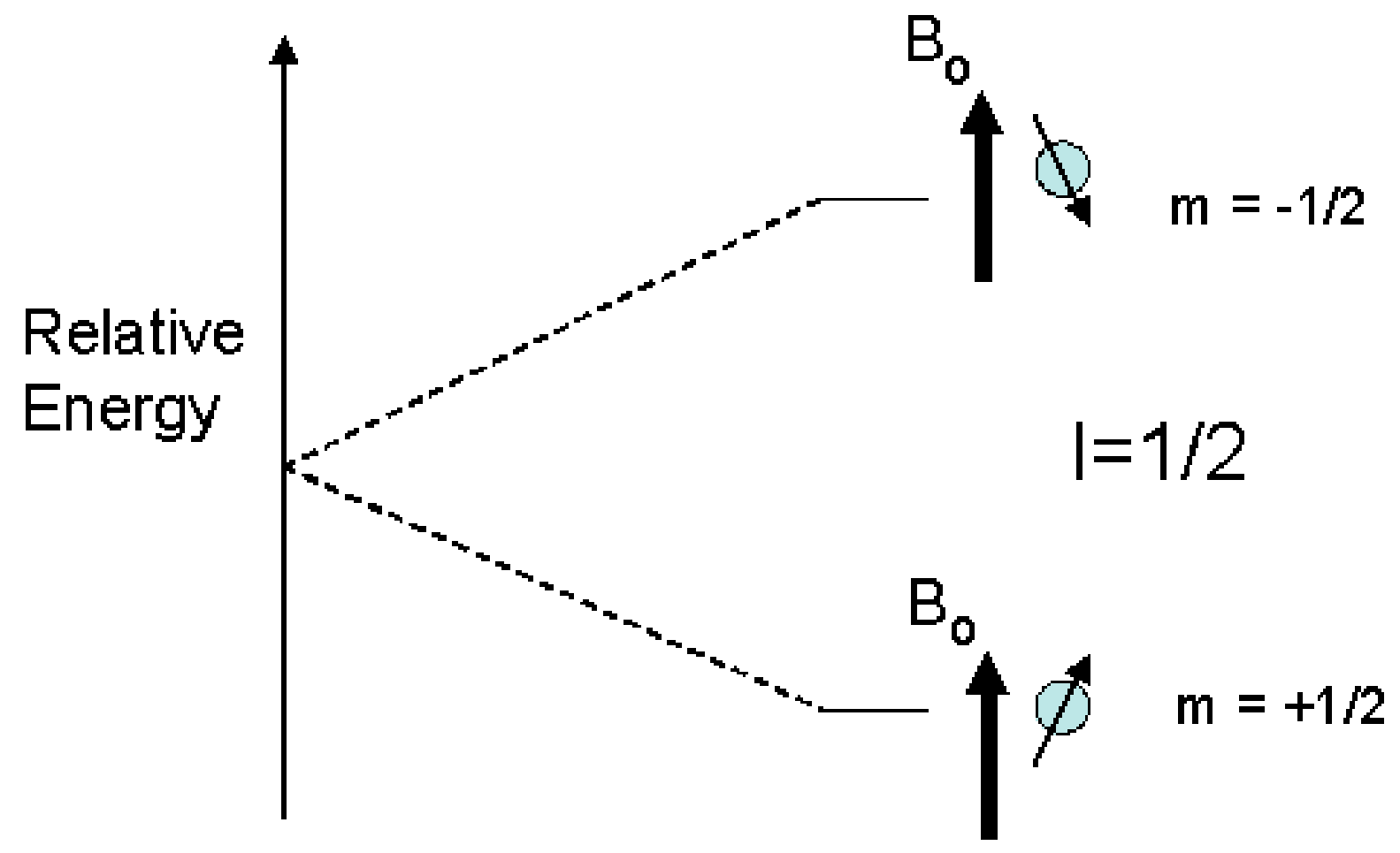

\section{Increasing Magnetic Field Strength}

Figure 1-10. When nuclei are exposed to an applied magnetic field, they align with the field in the alpha-spin state or against the applied field in the beta-spin state. The stronger the applied magnetic field is, the larger the energy gap is between the alpha and beta states. (Source: Adapted from process-nmr.com) 


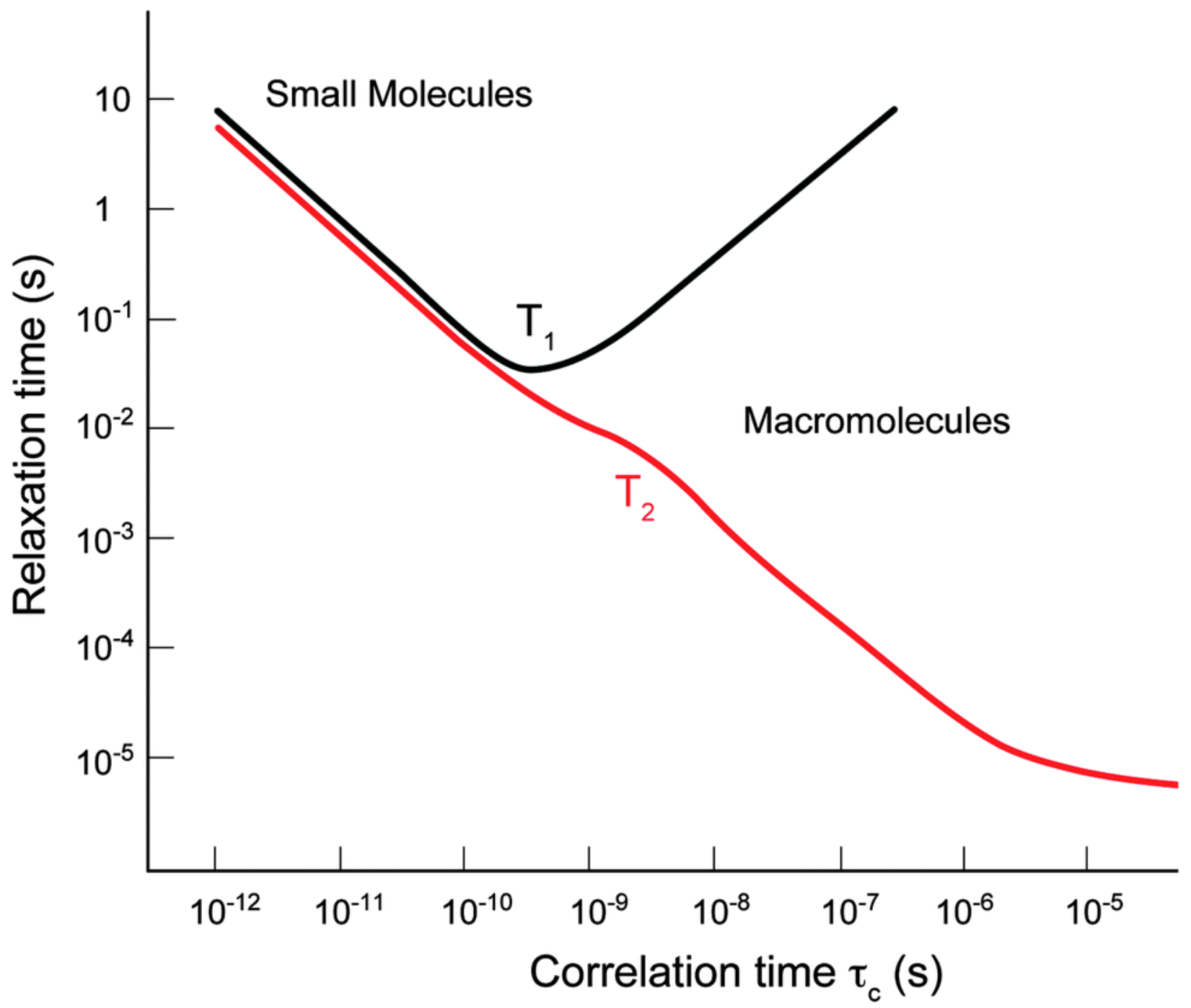

Figure 1-11. Shows the expected $\mathrm{T}_{1}$ and $\mathrm{T}_{2}$ values based on the size of a molecule. (Source: Adapted from R. Keshari, K.; M. Wilson, D. Chemistry and Biochemistry of 13 C Hyperpolarized Magnetic Resonance Using Dynamic Nuclear Polarization. Chemical Society Reviews 2014, 43 (5), 1627-1659.) 
$\mathrm{T}_{2}$ is the relaxation in the $\mathrm{x}-\mathrm{y}$ plane and is the loss of phase coherence among nuclei. During the scan, a $90^{\circ}$ pulse is applied and the nuclei spins are aligned in one direction but due to the inhomogeneity in the magnetic field or the interactions between the spins without energy transfer to the lattice the orientation is lost. If two similar nuclei have the same precession rates then the magnetic field of each nucleus can interact and cause an interchange of states so the excited nuclei relax and relaxed nuclei are excited. However, there is no net change in the spin state population. ${ }^{133}$ When the applied magnetic field varies from nucleus to nucleus, it can cause broadening because a band of frequencies is absorbed instead of a single one. Broadening could also be due to the other magnetic nuclei whose spins create local fields that could enhance or reduce the applied magnetic field. $T_{2} \leq T_{1}$ because the $\alpha$ and $\beta$ states naturally return to the $z$-plane causing the loss of magnetization in the $x-y$ plane.

Electrons from surrounding atoms have their own magnetic field known as diamagnetic currents that oppose the external magnetic field. The diamagnetic currents can reduce (shield) or enhance (deshield) the effect of the magnetic field on the proton. The shielding effect increases with increased electron density and higher atomic numbers because more electrons orbit the nucleus. Neighbors that can increase or decrease the electron density also affect it. The chemical shift, $\delta$, can be calculated using the absolute frequency, $v$, and the Larmor frequency, $\mathrm{v}_{0}$ and is in parts per million. ${ }^{93}$

$$
\delta=10^{6}\left(\mathrm{v}-\mathrm{v}_{0}\right) / \mathrm{v}_{0}
$$

A spectrum of a molecule will have a resonance for each nucleus in a different chemical environment. If two separate nuclei experience the same chemical environment, only one peak will appear but with a higher integration. 
A valuable piece of information that can be obtained from NMR spectra is the spin-spin splitting. Spin-spin splitting occurs when protons are bonded to adjacent atoms and have different chemical shifts. The magnetic field of the neighboring proton disturbs the external magnetic field causing an enhancement or a reduction depending on if the proton is in the $\alpha$ or $\beta$ state. Since the nuclei are nearly 50:50 in the $\alpha: \beta$ state, the proton resonance is split in half producing a doublet. ${ }^{133}$ If there is more than one neighboring proton, the splitting pattern can be more complex due to all the possible orientations of the protons. The ratio for the intensity of the splitting pattern follows Pascal's triangle. When a proton has two neighboring protons, there is a triplet splitting pattern with an intensity of 1:2:1 where the center peak has double the intensity of the two outer peaks. The spin-spin interactions can also be used to determine stereochemistry of molecules by measuring the strength of the interactions. The strength is measured by the $\mathrm{J}$ value $(\mathrm{Hz})$, which is the peak separation within the splitting pattern. ${ }^{133}$

The chemical shift can also be affected by hydrogen bonding. When hydrogen bonding occurs, the chemical shift increases because the proton is deshielded. ${ }^{135}$ Hydrogen bonding can affect the chemical shift of $\mathrm{OH}$ and $\mathrm{NH}$ protons. They can also undergo proton exchange. ${ }^{133}$ Depending on the solvent used, it can hydrogen bond with hydroxyls or amines creating changes in the chemical shift of the protons. When deuterated chloroform is used as the solvent, no H-bonding occurs with the solvent. However, if a solvent is a strong hydrogen bond acceptor, such as DMSO, an exchange of $\mathrm{OH}$ protons occurs causing the signal to shift downfield. If $\mathrm{D}_{2} \mathrm{O}$ is used as the solvent, no peak will appear because the $\mathrm{OH}$ can be transferred to the lone pair of the solvent.

\section{Previous NMR Applications}


NMR spectra provide information on the interactions between molecules through several methods. Simple ${ }^{1} \mathrm{H}$ NMR can be used to observe the change in chemical shift when a ligand, such as glucose, is titrated into a protein solution and determine the binding affinity. The changes in chemical shifts can indicate interactions. ${ }^{136}$ Isotope labeling in heteronuclear single quantum coherence (HSQC) can observe interactions based on the chemical shift as well. $\mathrm{T}_{1}$ and $\mathrm{T}_{2}$ measurements can be used to infer molecular interactions through peak broadening and lower $\mathrm{T}_{1}$ values. ${ }^{137}$

NMR spectroscopy has been used in this project used to study interactions between vitreal components that could be responsible for vitreal liquefaction.

\section{REASONS FOR THIS PROJECT}

The VH occupies $80 \%$ of the ocular volume and has important functions such as the maintenance of the ocular volume and its transparency by blocking the passage of large compounds that would interfere with vision. The compositional/structural changes that take place as the gel-like structure of the VH slowly turns into liquid over time need further study. Liquefaction is detrimental to ocular health because it can cause serious diseases such as retinal detachment.

To our knowledge, the potential role(s) that PLs may play in liquefaction have yet to be studied. Our long-term goal is to determine the structural/conformational alterations brought by compositional changes in PLs and their metabolites from peripheral tissues. This research will help us gain a deeper understanding of the mechanisms of liquefaction at the molecular level, the understanding of interactions between vitreal components, and the effect species migrating in from retinal tissue have on the biomatrix. This knowledge 
will be helpful for the development of future approaches to prevent vitreal and retinal diseases.

Chapters 2 and 3 focus on mapping the regional distribution of PLs and glucose in mammalian VHs to better understand the regional composition and the role they have on liquefaction using MALDI/MS. Based on the data collected, chapter 4 uses NMR and MALDI/MS to explore the interactions of HA-PLs and HA-glucose. It explores the role(s) that PLs and glucose could have in disrupting the gel-like HA-collagen-water hydrogen bonded network and possible effects of liquefaction. Chapter 5 concludes with a summary of the work and future directions of this project. 


\section{CHAPTER 2}

\section{REGIONAL DISTRIBUTION OF PHOSPHOLIPIDS IN PORCINE VITREOUS}

\section{HUMORS}

\section{INTRODUCTION}

The $\mathrm{VH}$ is located between the retina and the lens of the eye and occupies $80 \%$ of the ocular volume. A highly hydrated (98\% water) network of collagen and HA leads to its gel-like consistency. It is believed that the collagen creates the framework and the highly hydrated HA fills the spaces thus adding stability to the network. In addition,

proteoglycans, PLs, other glycosaminoglycans, and metabolites are present in the VH. ${ }^{1,38}$ Indeed, it is considered as a 'repository' of compounds derived from adjacent tissues. ${ }^{59}$

As the VH ages, the gel-like structure of the VH is slowly lost. This process is referred to as liquefaction and it has been reported to start as early as age four in humans. After seven decades of life, $50 \%$ of the $\mathrm{VH}$ is liquefied. ${ }^{59}$ There are many hypotheses on how or why the VH liquefies. For example, it has been proposed that enzymatic activity disrupts the collagen network; type IX collagen breaks down causing type II collagen to stick to itself; HA breaks down; collagen and HA interactions are hindered because macromolecules change the conformation of HA. ${ }^{23,27,47,59}$ Liquefaction is a serious problem because it can lead to ocular diseases such as posterior vitreous detachment, macular hole formation, and retinal detachment. ${ }^{48}$ Although much information is 
available regarding the $\mathrm{VH}$, little is known about the specific molecular changes that affect the composition and structure of the $\mathrm{VH}$ and lead to its liquefaction.

The VH has been studied using dark-field slit microscopy as an in-vitro technique that showed the structure of collagen and age- related changes on the overall vitreous structure. NMR spectroscopy was applied in vitro to study the VH. From the decrease in the longitudinal and transverse relaxation times, Aguayo et al. showed a reduction in the VH viscosity when collagenase was added. ${ }^{78}$ However, the addition of hyaluronidase led to minor changes whereas ascorbic acid did not. Dynamic light scattering has been used to observe changes in the level of heterogeneity and particle size in diabetic vitreopathy. Optical coherence tomography has also been applied to measure the size and distance between structures within the $\mathrm{VH} .{ }^{77}$

The presence of minute amounts of PLs in the VH was reported as early as 1954 by D'asaro. ${ }^{30}$ Due to the extremely low PL concentrations, very sensitive techniques are needed to detect PLs and other constituents of the VH. In the past, PLs have been quantified in ocular tissues (lens and retina) using ${ }^{31} \mathrm{P}$ NMR spectroscopy. ${ }^{80,83}$ Unfortunately, this approach does not provide the sensitivity needed for the regional quantification of PLs composition of VHs. At this time, MALDI/MS has been successfully applied to study the distribution of lipids, proteins, and other metabolites throughout the retina and lens. ${ }^{84-90}$

MALDI is a soft ionization technique in which a matrix compound is mixed with the sample. This matrix is present in excess relative to the analytes and absorbs at the wavelength emitted from the laser source. The matrix and analytes are placed on a plate where they co-crystallize. This metal plate is inserted into the ionization chamber held at 
high vacuum levels. ${ }^{108}$ A laser is fired, the matrix absorbs the energy that is then transferred to the analyte(s). Sublimation of the matrix and analyte occurs causing their desorption and ionization. ${ }^{93}$ Neutral species can be protonated or cationated and lead to the presence of cations from the parent molecules. ${ }^{138}$ Negative-ion spectra can also be acquired and the peaks correspond, most often, to the deprotonated analytes. ${ }^{138}$

The matrix choice is of utmost importance for MALDI/MS. Good results have been reported with the use of PNA for the analysis of lens PLs in-vitro and in-situ. ${ }^{85,122}$ Although it has been successfully used in ambient-pressure MALDI, PNA sublimes under high vacuum conditions and while it can be used for short (20-30 min) acquisition times, this matrix is not suitable for lengthy imaging experiments. ${ }^{139,123,124,125}$ This report focuses first on the development/optimization of the analytical approaches for measuring in vitro and ex situ the regional distribution of PLs in porcine VHs. Secondly, by comparison of the compositions of the anterior, central and posterior vitreous regions with those corresponding to the lens and retina, the possible sources of PLs in the VH will be assessed. To our knowledge, the potential role(s) that PLs may play in liquefaction have yet to be studied. Determining regional compositional differences in the $\mathrm{VH}$ will contribute to our understanding of the molecular changes that lead to $\mathrm{VH}$ liquefaction.

\section{EXPERIMENTAL}

\section{Chemicals}

Para-nitroaniline (PNA), chloroform $\left(\mathrm{CHCl}_{3}\right)$, and methanol $(\mathrm{MeOH})$ were purchased from Aldrich Chemical Co., Inc. (Milwaukee, WI). 


\section{Tissue source}

Porcine eyes were collected from a local abattoir and immediately dissected or frozen at 4 C. Although the actual age of each eye was not given, the range was between 4 and 12 months.

\section{Matrix application}

The matrix used for the analysis of PLs was para-nitroanaline (PNA) dissolved in methanol (26 mg PNA/mL MeOH). Several approaches were tested for the matrix/sample application, as listed below.

'Dried-Droplet Method': A 1-mL aliquot of PNA solution was spotted directly on top of either a clean MALDI plate (blank) or a sample previously spotted on the stainless-steel MALDI plate.

'Premix Method': A PL sample (extract or control) and the PNA solution were premixed and spotted on a MALDI plate.

'Dip Method': The entire MALDI plate was dipped in a dish filled with PNA solution (26 mg PNA/mL MeOH). The solvent was evaporated using a stream of cold air from a blow dryer.

'Sublimation Method': A dish with PNA crystals was placed on a hot plate and a MALDI plate was placed 2 inches above the dish. Heat was applied (low setting) for 20 min or until all PNA was sublimed onto the MALDI plate with or without (blank) a stamped sample.

'Recrystallization Method': (Adapted from Duenas et al., 2016 ${ }^{140}$ ). A plate coated with

sublimed PNA was placed in a Petri dish (See Fig. 1). A piece of tissue (Kimwipes ${ }^{\circledR}$ (Kimberly-Clark, Roswell GA)) cut in half was wetted with $1 \mathrm{~mL} \mathrm{MeOH:CHCl} 3$ (1:2) 
and placed in the bottom of a Petri dish and sealed with tape to form a solvation chamber. The chamber was placed in a warmed oven at $50 \mathrm{C}$ for $2 \mathrm{~min}$.

\section{Ex-situ analysis}

'Core Stamp Method': A porcine eye was frozen in liquid nitrogen. Then, the VH was separated from the rest of the eye by removing the sclera, retina and lens. A copper tube (1/4 inch diameter) with a jagged edge was introduced into the frozen $\mathrm{VH}$ to collect the 'core' of the VH. The tube was removed from the $\mathrm{VH}$ and the frozen core was pushed through the tube slowly and stamped repeatedly at different locations on the MALDI plate. Because the edges of each print were exposed to the tube walls that touched other areas of the $\mathrm{VH}$, only the center of each stamp was analyzed. PNA droplets $(1 \mathrm{~mL})$ were spotted onto the center of the stamps.

'Stamp Method': A frozen porcine ocular globe was sliced along the sagittal plane and the $\mathrm{VH}$ was isolated. One of the halves was taken and placed on a MALDI plate for $20 \mathrm{~s}$ and removed. Longer 'stamp' times caused layers that were too thick for analysis. The MALDI plate was either dipped in PNA or left clean for the stamping process. After stamping, PNA was sublimed or spotted onto the stamp if the dip method was not used.

\section{In-vitro analysis}

The VH was obtained in the same way as in the stamp method but it was then sectioned to collect the posterior, central and anterior regions. Once the three sections were isolated, a thin layer of the outermost regions was discarded to remove residual tissue form the adjacent zones. The remaining section was then analyzed. Each section was

extracted using the Bligh and Dyer method ${ }^{141}$ by adding $3.75 \mathrm{~mL}$ of $\mathrm{MeOH}: \mathrm{CHCl}_{3}(2: 1)$ per gram of tissue. After sonication, $1.25 \mathrm{~mL}$ of $\mathrm{CHCl}_{3}$ and $1.25 \mathrm{~mL}$ of $\mathrm{H}_{2} \mathrm{O}$ were added 
per gram of tissue. Other extraction methods used included extraction with just $\mathrm{MeOH}$ or with $\mathrm{MeOH}: \mathrm{CHCl}_{3}$ (1:2). ${ }^{142}$ The organic layer of the extract was mixed with solid PNA to achieve a matrix concentration of $26 \mathrm{mg} / \mathrm{mL}$. The solution containing the organic extract and PNA was spotted directly on a MALDI plate and analyzed.

\section{Data acquisition}

A matrix-assisted laser desorption ionization time of flight/mass spectrometer (MALDITOF/MS, Voyager Biospectrometry DE workstation, Applied Biosystems, Foster City, CA) with a nitrogen laser that emits at $337 \mathrm{~nm}$ was used to analyze all samples. Positive and negative-ion spectra were collected in the reflector mode. An accelerating voltage of $20 \mathrm{kV}$ was applied after an extraction delay time of $75 \mathrm{nsec}$. The laser energy was $70 \pm 5$ $\mathrm{mJ}$ and the pulse rate $3 \mathrm{~Hz}$. The laser intensity was adjusted to result in minimum fragmentation and high sensitivity. The mass range of $50-1200 \mathrm{~m} / \mathrm{z}$ was used and 75 shots were averaged per spectrum in both positive and negative mode. Data Explorer software version 4.8 (Applied Bio- systems, Foster City, CA) was used to analyze the data.

\section{RESULTS}

\section{Experimental optimization}

The solvent systems tested to dissolve the matrix, PNA, were those used for extracting PLs from tissue: $\mathrm{MeOH}_{\mathrm{CHCl}}$ (1:1), $\mathrm{CHCl}_{3}, \mathrm{MeOH}: \mathrm{H}_{2} \mathrm{O}$ (1:1), and $\mathrm{MeOH}$. As seen in Fig. 2-1, when PNA was dissolved in $\mathrm{MeOH}$ only, intense interference peaks were present. The peaks were also observed when $\mathrm{H}_{2} \mathrm{O}: \mathrm{MeOH}$ (1:1) was used. The interference peaks are believed to be due to polyethylene glycol (PEG) chains since the 
average experimental difference of $\mathrm{m} / \mathrm{z}$ values between adjacent peaks is $44.022 \pm 0.013$. The theoretical value for the $-\left(\mathrm{OC}_{2} \mathrm{H} 4\right)$ - repeating unit is 44.026 , well within the observed experimental range. However, PEG-related peaks previously reported in

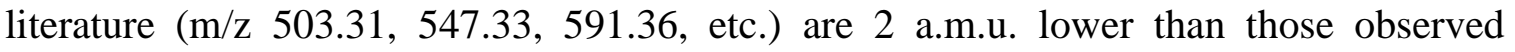
herein. ${ }^{143}$ Glass vials and pipettes were used to prevent contamination from the plastic material but the interferences were still detected. Studies are underway to determine the source of the contaminants. These interferences were greatly reduced by using a less polar solvent, such as $\mathrm{CHCl}_{3}$ (Fig. 2-1b). However, PNA does not fully dissolve in $\mathrm{CHCl} 3$. To overcome this problem, a 1:1 mixture of $\mathrm{MeOH}: \mathrm{CHCl}_{3}$ was tested. This allowed for suppression of the interference peaks and complete solubility of PNA as seen in Fig. 2-1d.

Unfortunately, other interferences appeared at $\mathrm{m} / \mathrm{z}$ values of $647.49,663.48$, and 685.47 when using $\mathrm{CHCl}_{3}$ (Fig. 2-2). These peaks were initially thought to be due to sphingomyelin (SM) $[\mathrm{SM}(18: 0 / 13: 0)+\mathrm{H}]^{+}$and $\mathrm{SM}[(18: 1 / 12: 0)+\mathrm{H}]^{+}$with $\mathrm{m} / \mathrm{z}$ values of 663.54 and 647.51, respectively. However, literature searches revealed that these peaks had been observed in previous studies and are related to tris(2,4-ditert-butylphenyl) phosphite, referred to as Irgafos $168 .{ }^{144}$ The theoretical $\mathrm{m} / \mathrm{z}$ values for its protonated, sodiated and potassiated adducts are $647.46,663.44$ and 685.42 . Irgafos is a processing stabilizer and antioxidant that is present in Eppendorf tubes. The solubility of Irgafos 168 is $25 \mathrm{mg} / \mathrm{mL}$ in chloroform but is insoluble in water $(0.00009 \mathrm{mg} / \mathrm{mL}) .{ }^{145}$ This is the reason for which the interferences were only observed when chloroform was used as a solvent in the Eppendorf tube. When glass vials were used, these peaks were no longer detected. 

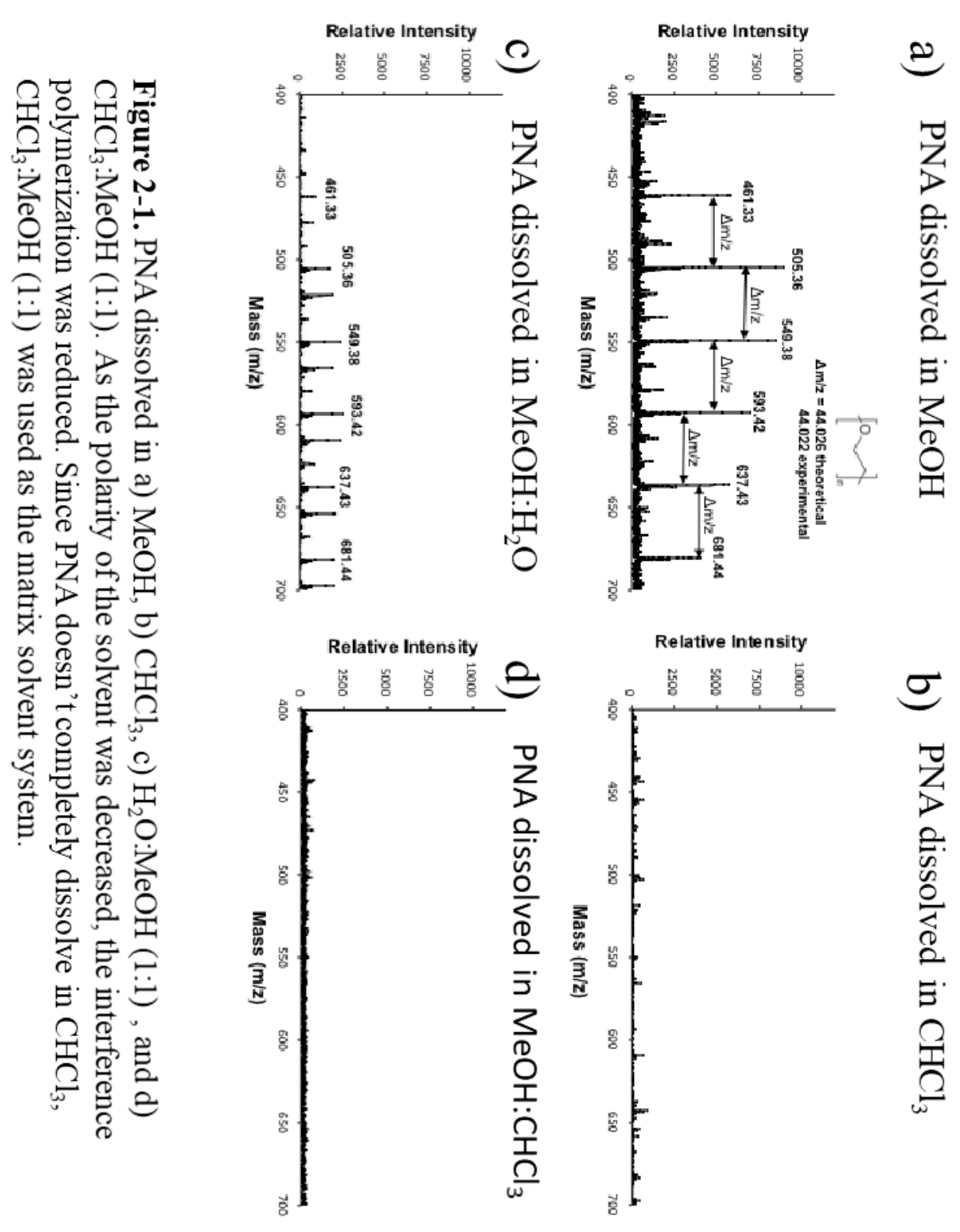


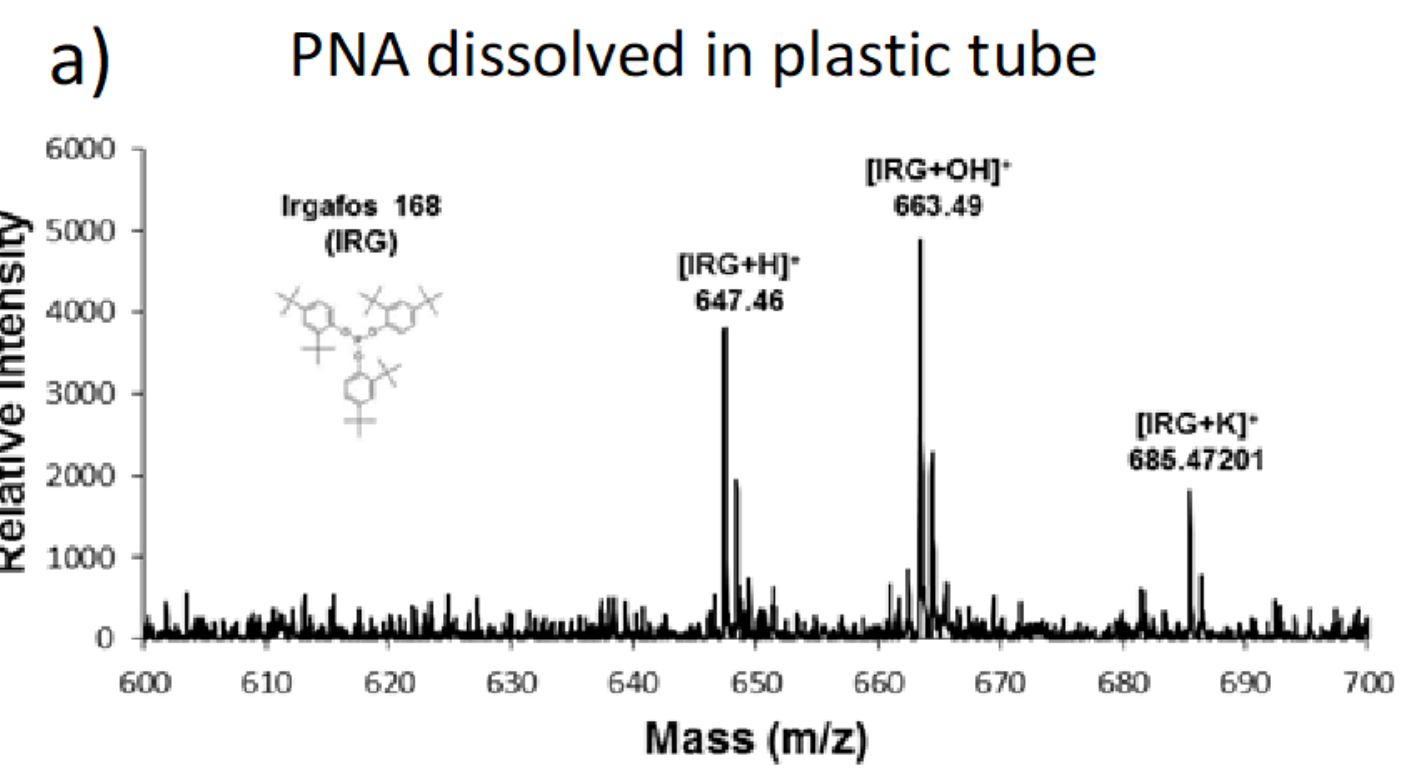

b)

PNA dissolved in glass vial

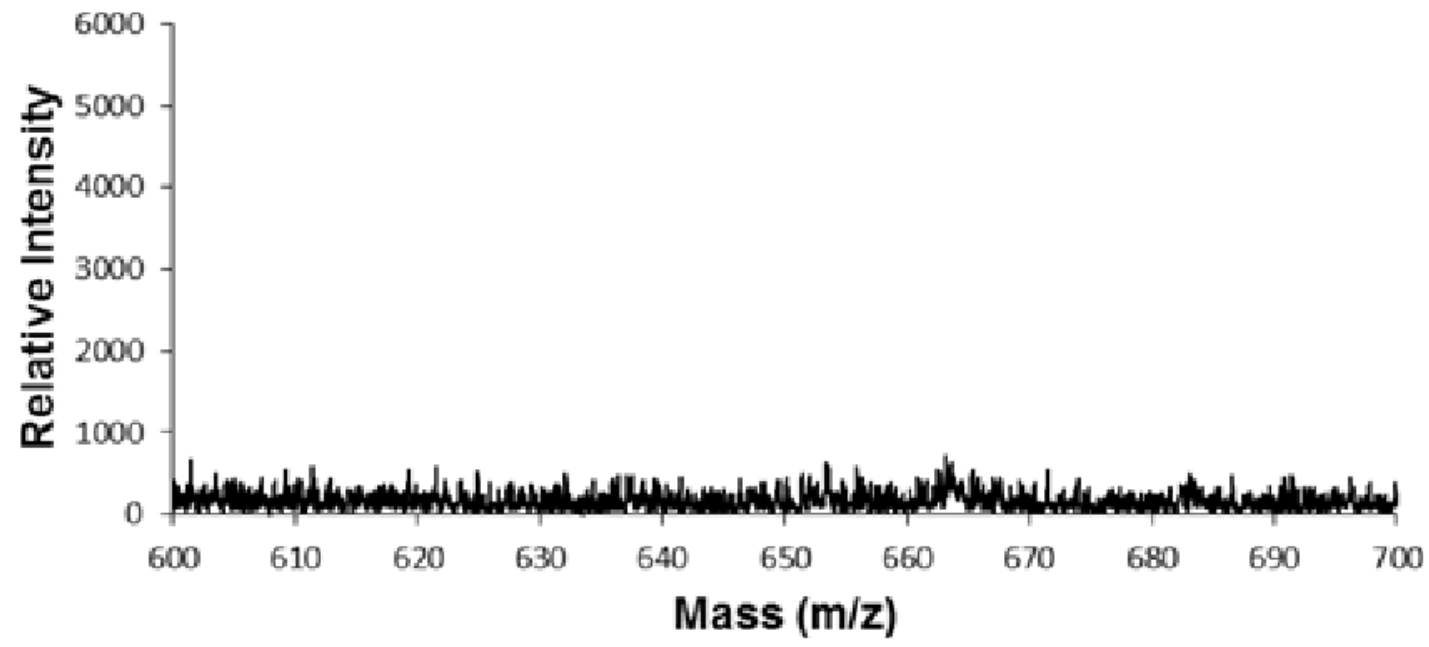

Figure 2-2. PNA dissolved in a) plastic Eppendorf tube and b) glass vial. Since the solvent system of $\mathrm{CHCl}_{3}: \mathrm{MeOH}(1: 1)$ was used with the matrix, the $\mathrm{CHCl}_{3}$, reacted with the plastic tube causing Irgafos 168 to appear in the spectra. Once the plastic was replaced with glass, the peaks no longer appeared. 


\section{In-vitro results}

Experimental Aspects:

After the PNA conditions were optimized, several extraction methods for in-vitro studies were explored. They include the use of $\mathrm{MeOH}: \mathrm{CHCl}_{3}$ (1:2), $\mathrm{MeOH}$ only, as well as the Bligh and Dyer (B\&D) method in which a biphasic system, $\mathrm{MeOH}: \mathrm{CHCl}_{3}: \mathrm{H}_{2} \mathrm{O}$ (2:2:1), is used. Interestingly, but not surprisingly, different extraction approaches were more effective for each of the various ocular tissues. Overall, the sensitivity observed in the spectra for lens and retinal $\mathrm{MeOH}$ extracts was higher than that for the $\mathrm{MeOH}: \mathrm{CHCl} 3$ extracts, but the compositional trends were the same. In addition, a few PLs undetectable in $\mathrm{MeOH}$ extracts of $\mathrm{VH}$ s could be detected in the $\mathrm{MeOH}: \mathrm{CHCl}_{3}$ extracts. The sensitivity obtained for the VH B\&D extracts surpassed that of the other two extraction methods. For example, the signal-to-noise ratio (S/N) for the peak at m/z 734 was 730:1 (B\&D) but only 100:1 and 130:1 in the $\mathrm{MeOH}: \mathrm{CHCl}_{3}$ and $\mathrm{MeOH}$ extracts, respectively. More SM species were also detected using the B\&D method, which was therefore chosen to explore the regional PL composition of porcine VHs.

Regional PL distribution:

The PLs detected in the lens, VH regions and retina are listed in Table 2-1. No peaks related to PL metabolites or fragments were detected below $\mathrm{m} / \mathrm{z}$ of 700 suggesting that these species are not present or are below detection limits in young porcine VHs. Positive-ion mass spectra of the $\mathrm{MeOH}: \mathrm{CHCl}_{3}$ extracts of porcine $\mathrm{VH}$ showed several phosphatidylcholine (PC) species, with PC(34:1) in highest abundance, followed by PC(32:0) (Fig. 2-3). In the retina extract, high levels of $\mathrm{PC}(34: 1)$ were observed and SM(16:0) was also detected. The lens extract also showed PC species. PC(32:0) was the 


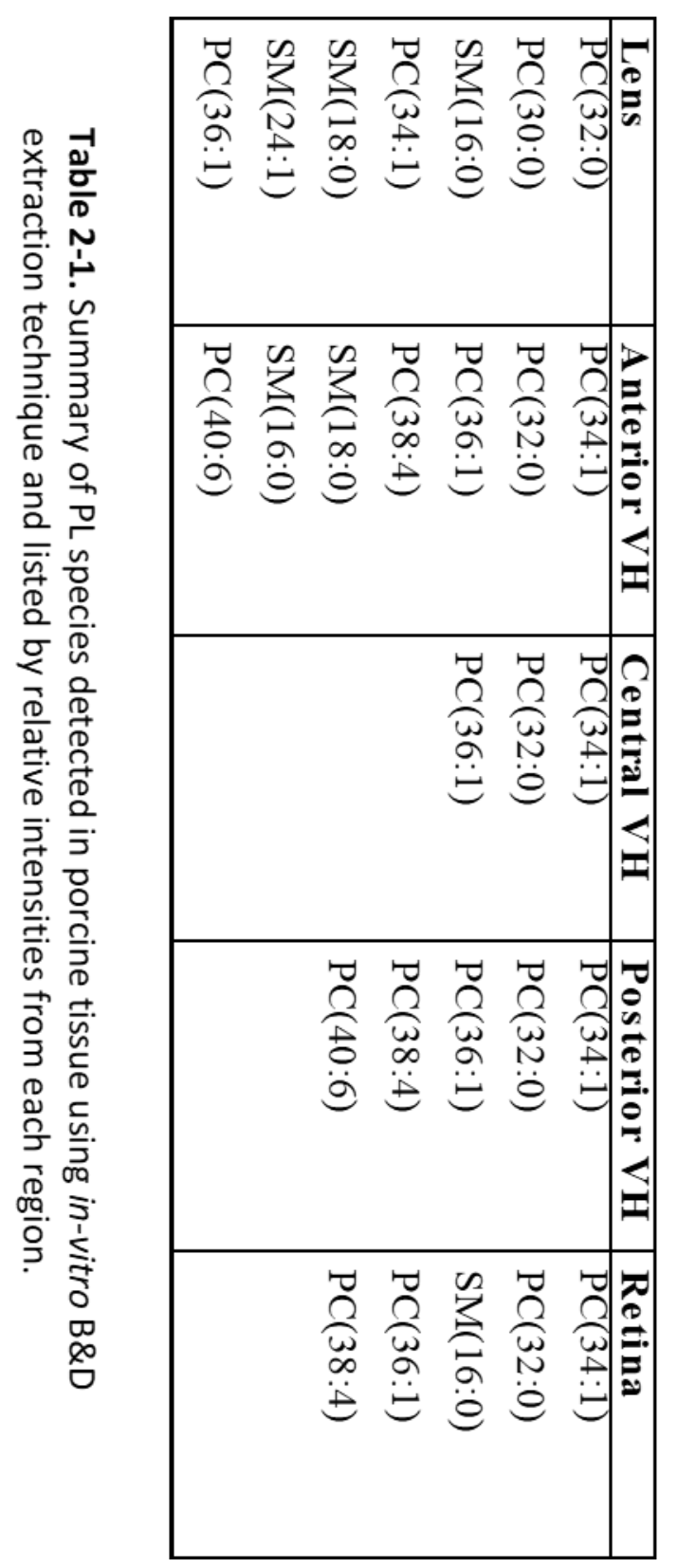




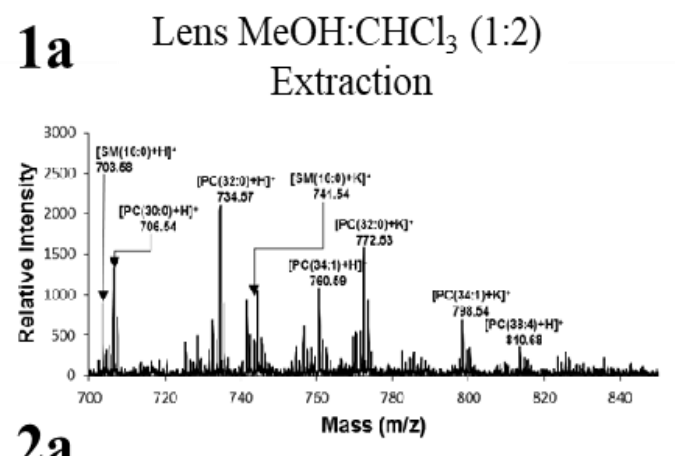

2a

VH MeOH: $\mathrm{CHCl}_{3}(1: 2)$ Extraction
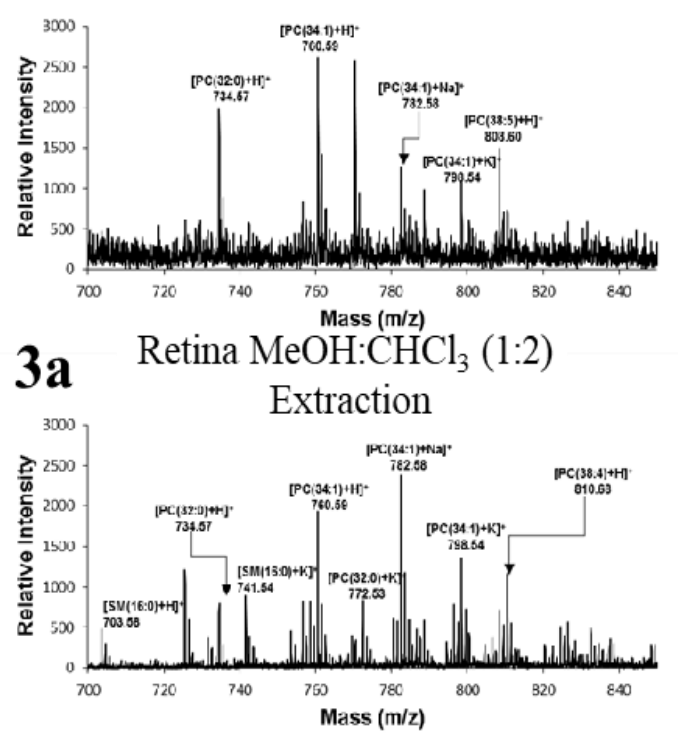

$\mathbf{1 b}^{*}$ Lens $\mathrm{MeOH}$ Extraction

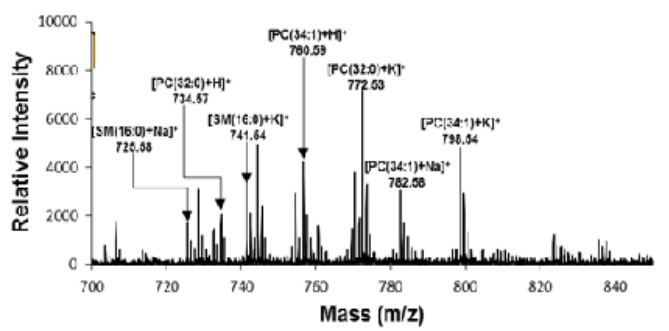

2b VH MeOH Extraction

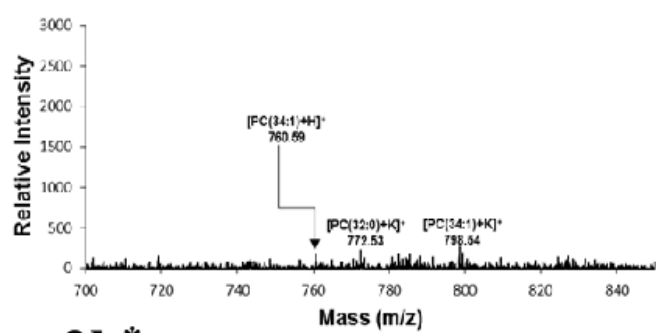

3b* Retina MeOH Extraction

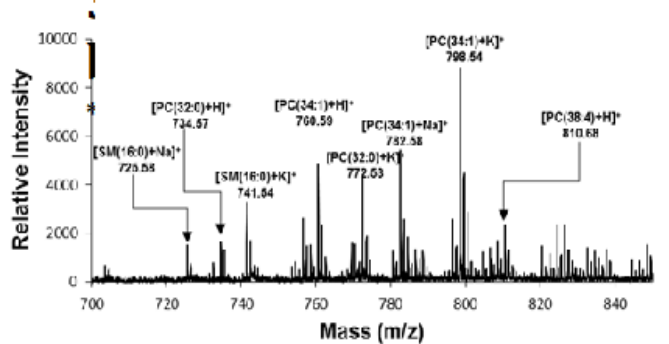

Figure 2-3. Extraction of PLs using $\mathrm{MeOH}: \mathrm{CHCl}_{3}$ (1:2) (a) and $\mathrm{MeOH}$ only (b) on the 1) lens, 2) $\mathrm{VH}$, and 3) retina. *Note that the axis for $1 \mathrm{~b}$ and $3 \mathrm{~b}$ are in a different scale due to the high relative intensity of the PL peaks. The extraction method using $\mathrm{MeOH}: \mathrm{CHCl}_{3}$ (1:2) and $\mathrm{MeOH}$ only have previously been used to study retinal and lens tissue. However, these methods do not prove to be ideal for studying the VH. 
most abundant, and PC(30:0) was also observed.

As seen in Fig. 2-4, there is a greater abundance of PCs in the posterior region of the VH. The highest levels were seen for PC(34:1) followed by $\mathrm{PC}(32: 0)$. The anterior region showed the presence of $\mathrm{SM}(16: 0)$ as well as the PC species. However, their abundance was lower relative to the posterior region. Negative-mode mass spectra were also collected for the all the extracts. PL related peaks were not detected in the negativeion mass spectra of $\mathrm{VH}$ extracts. This is believed to be due the relatively minute levels of PLs in porcine VHs and the inherently lower sensitivity of negative-ion spectral data. However, the lens and retina samples extracted using the B\&D method showed a variety of species. The lens extract showed high levels of phosphatidylethanolamine plasmalogen (PEp) (36:2) followed by PEp(34:1). In addition, phosphatidylserine (PS) (36:1) and PS(34:1) as well as phosphatidylinositol (PI) (36:2) (Fig. 2-5). The peak corresponding to $\operatorname{PEp}(38: 4)$ was the most intense followed by those of PI(38:4) and PS(36:1). PEp(36:2), PEp(34:1), PEp(40:4), and PS(38:4) were also present but in lower relative levels in the retina extract.

\section{Ex-situ results}

Experimental Aspects:

Several approaches were tested for the application of the matrix and sample onto the MALDI plate. Initially, the MALDI plate was dipped in PNA (26 mg/mL of MeOH) and the VH was stamped on top of the dried matrix. This approach showed little promise as only the potassiated species of $\mathrm{PC}(34: 1)$ and $\mathrm{PC}(32: 0)$ were detectable with relatively poor S/N (80:1 and 30:1, respectively). Secondly, PNA was sublimed on top of a dried 
a)

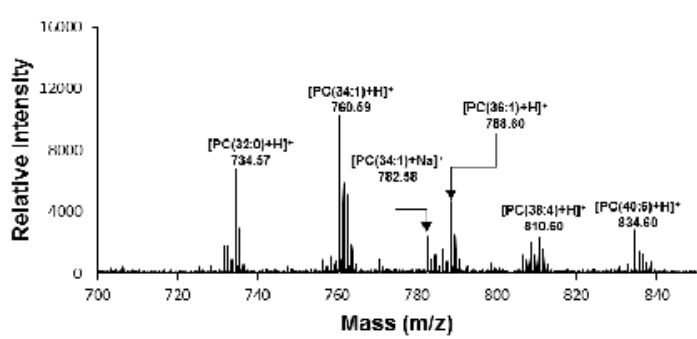

b)

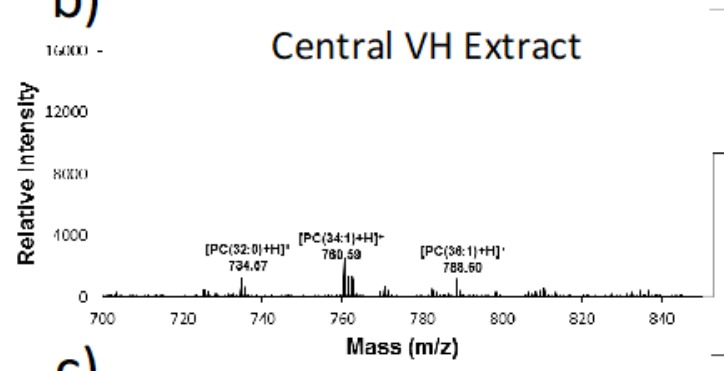

c)

Anterior VH Extract

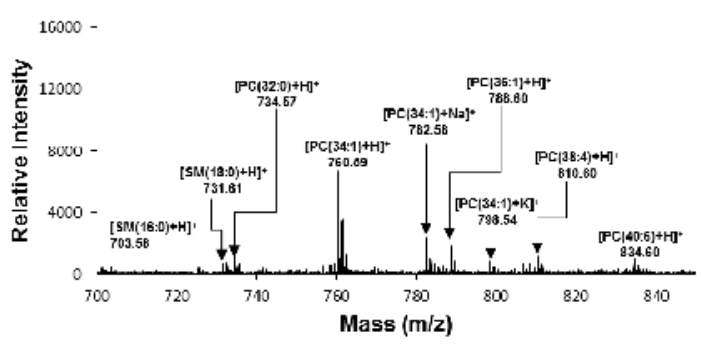

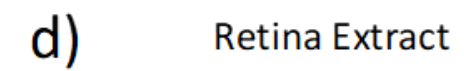

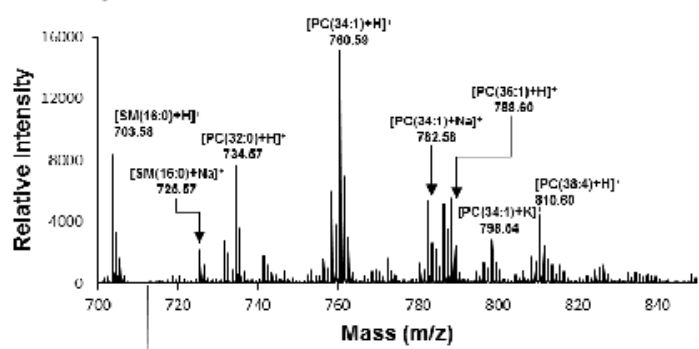

e)
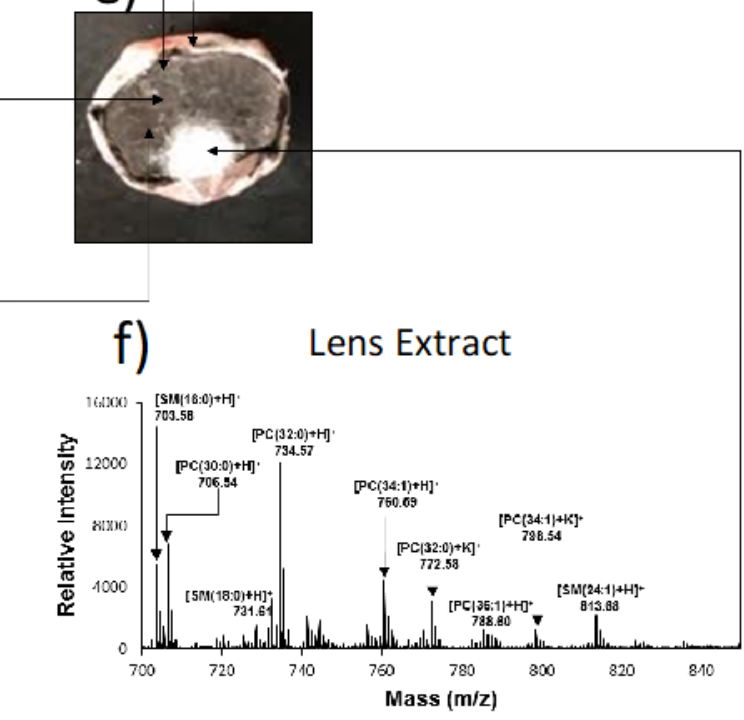

Figure 2-4. B\&D extraction of the a) anterior region of the $\mathrm{VH}, \mathrm{b}$ ) central region of the $\mathrm{VH}, \mathrm{c})$ posterior region of the $\mathrm{VH}, \mathrm{d}$ ) lens, and f) retina from e) frozen porcine eye. The B\&D extraction showed to be the best method for the analysis of PLs in the VH. The regional trends can be seen that the highest abundance of PLs are in the posterior followed by the anterior and the central regions. 
a)

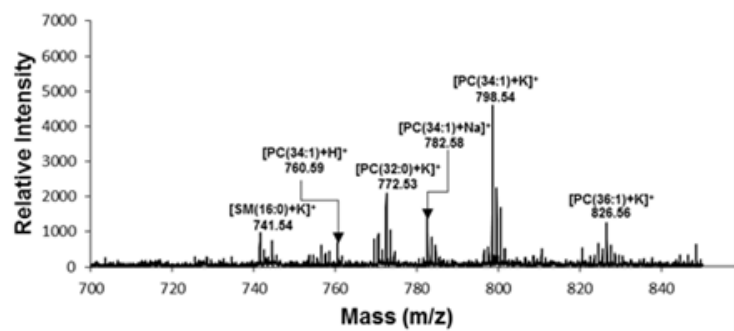

b)
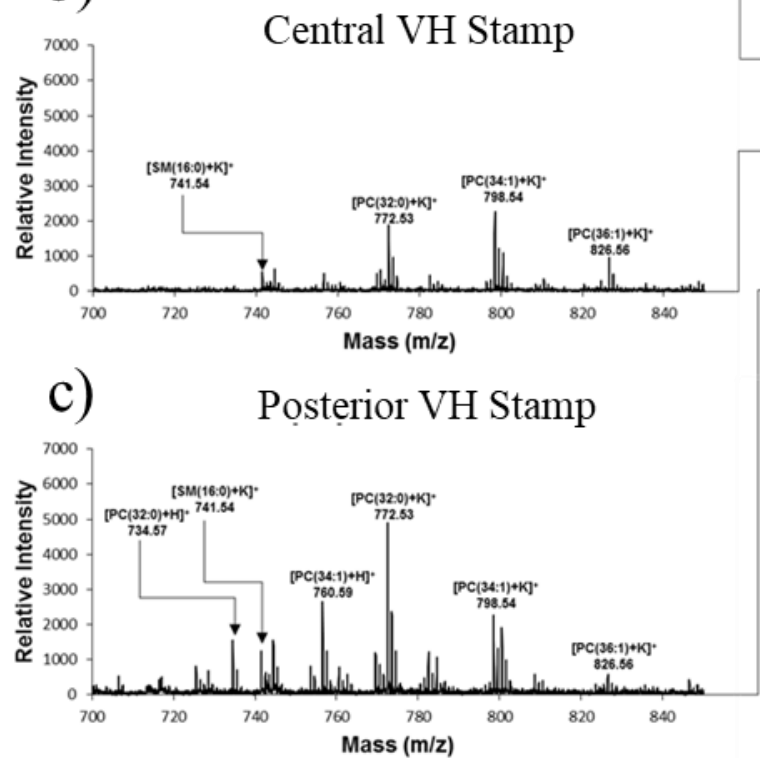

d) Lens Stamp

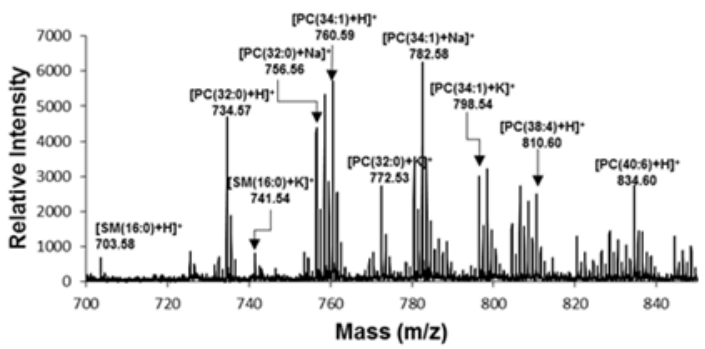

e)
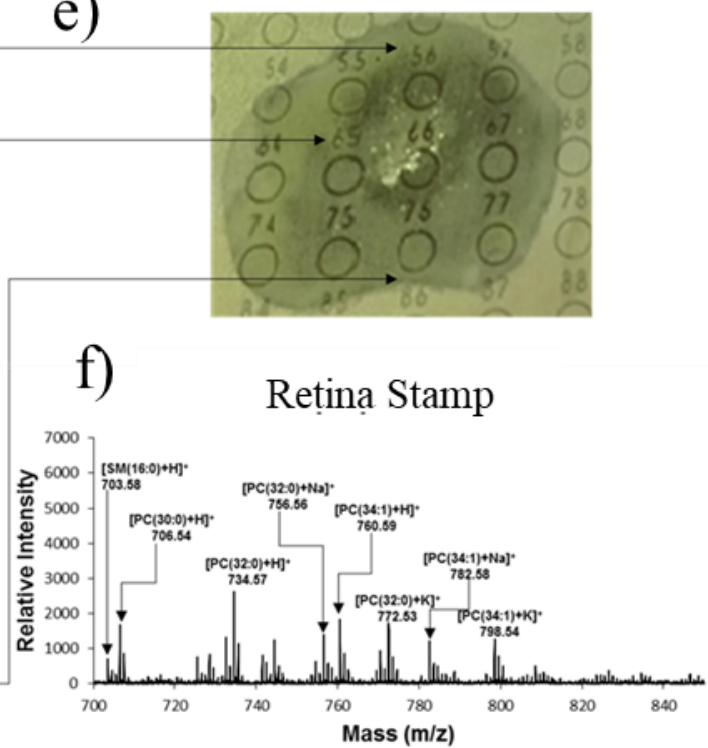

Figure 2-5. e) Imprint of porcine VH on a MALDI plate and regionally spotted with $0.100 \mathrm{uL}$ of PNA from the a) posterior $\mathrm{VH}, \mathrm{b}$ ) central $\mathrm{VH}, \mathrm{c}$ ) anterior $\mathrm{VH}, \mathrm{d}$ ) retina, $\mathrm{f}$ ) lens. The anterior VH stamp reflects similar species detected in the lens such as $\mathrm{SM}(16: 0)$ and $\mathrm{PC}(32: 0)$. The posterior region shares similarities with the retinal tissue with PC(34:1) being the most intense species in both. 
tissue stamp. This technique was not successful because the PNA did not co-crystallize with the dried tissue and sublimed once exposed to the vacuum environment of the MALDI before the acquisition could begin. A variation of this method was tested. ${ }^{140}$ After matrix sublimation onto the stamped sample, a recrystallization step was incorporated. Although more PL species were detected relative to the 'dip' method, the sensitivity was still poor. The S/N was 20:1 for PC(32:0) and 30:1 for PC(34:1). Lastly, the VH was stamped onto a clean MALDI plate but in this case, a 0.1-mL aliquot of PNA dissolved in $\mathrm{MeOH}: \mathrm{CHCl}_{3}$ was spotted on top of the dried VH stamp (Fig. 2-6). The resulting spot was $2 \mathrm{~mm}$ in diameter. The $\mathrm{S} / \mathrm{N}$ nearly tripled relative to the values seen with the dip method, going from 30:1 to $90: 1$ for $\mathrm{PC}(32: 0)$ and $80: 1$ to $210: 1$ for $\mathrm{PC}(34: 1)$ in the posterior region of the VH stamp.

It is believed that the solvent in the PNA droplet ( $\left.\mathrm{MeOH}: \mathrm{CHCl}_{3}\right)$ allowed the PLs to be 'extracted' from the biomatrix and detected more readily. The smaller aliquot of matrix applied to the tissue produced a homogeneous distribution unlike the larger aliquot.

Regional PL distribution:

The species present in the various regions of the $\mathrm{VH}$ as well as in the lens and retina are listed in Table 2-2. In the $\mathrm{VH}$, the most abundant $\mathrm{PL}$ in the anterior region was $\mathrm{PC}(32: 0)$ followed by $\mathrm{SM}(16: 0)$. In the posterior region, the peak due to $\mathrm{PC}(34: 1)$ was the most intense and the overall $\mathrm{S} / \mathrm{N}$ values were higher than those seen in the anterior and central regions. The central region contained PC(34:1) followed closely by PC(32:0). The retina and lens stamps showed that PC(32:0) was the most abundant species in the lens stamp, followed by $\mathrm{PC}(30: 0)$, which was not detected in the retina stamp. Overall, 

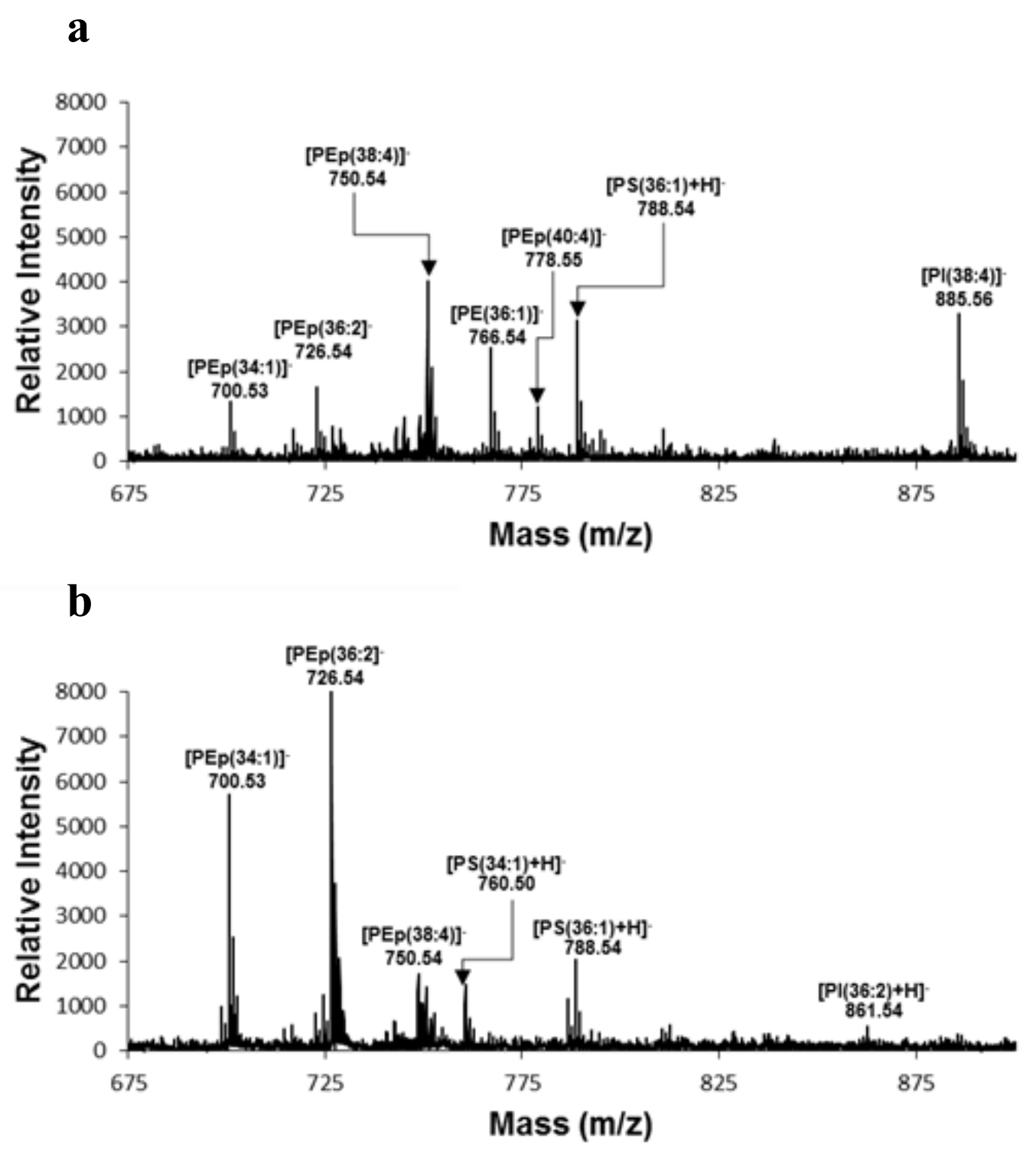

Figure 2-6. Negative mode of the a) retina and b) lens using the B\&D extraction method. A compositional variation can be seen from the different tissues. PS and PI are more abundant in the retina where PEp is the most abundant species in the lens. 


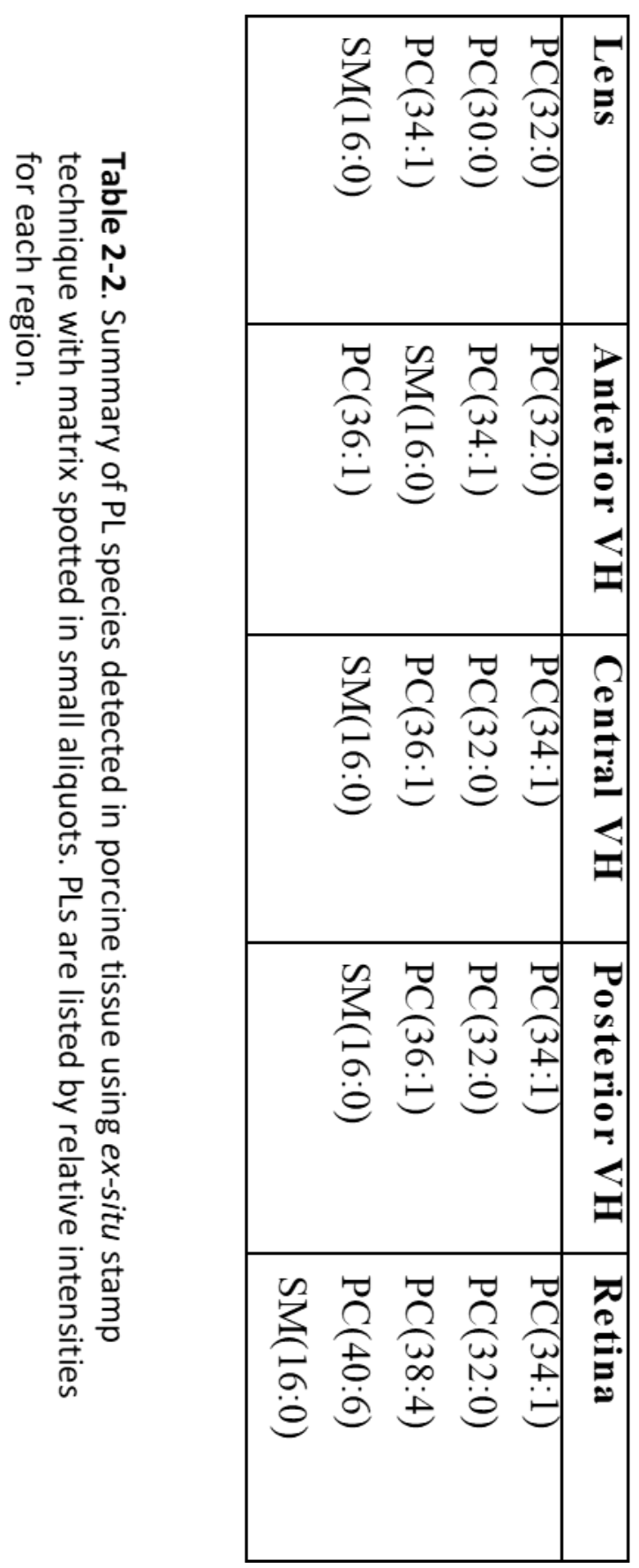


the $\mathrm{S} / \mathrm{N}$ for the PLs present in the lens were lower than those in the retina stamps for which PC(34:1) followed by PC(32:0) were the most abundant. SM(16:0) was detected in both the lens and retina stamps. Unfortunately, no PL peaks were detectable in the negative-ion spectra corresponding to the stamped tissues due to the minute PL amounts transferred to the MALDI plate and the lower sensitivity of negative-ion mass spectra.

\section{DISCUSSION}

\section{Analytical approaches}

Matrix Choice:

Although dihydroxybenzoic acid is an effective matrix for the MALDI/MS detection of relatively small molecules, such as phospholipids, PNA was shown to be a better choice for the PL analysis in porcine VHs. ${ }^{146}$ The best solvent system to dissolve the PNA was $\mathrm{MeOH}: \mathrm{CHCl}_{3}(1: 1)$ because it prevents the presence of interference peaks seen with just $\mathrm{MeOH}$ alone but still allows the solubilization of PNA, unlike when $\mathrm{CHCl}_{3}$ was used alone.

In-Vitro Experimental Optimization:

As shown in Fig. 2-3, while the best sensitivity was obtained for the methanolic extracts of the lens and retina, this was not the case for the $\mathrm{VH}$ extracts for which the $\mathrm{B} \& \mathrm{D}$ extraction provided the best results. The $\mathrm{B} \& \mathrm{D}$ extraction is a biphasic system and previous studies on the analysis of lens PLs showed the presence of sphingolipids in the interfacial layer. ${ }^{142}$ However, for the VH extracts, while significant amounts of PLs were detected in the organic layer, none were detectable in the interface.

Despite the mentioned changes in extraction efficiency, the type and relative 
abundance of PLs detected in the various extracts were comparable. Indeed, the overall trends observed in the comparison of lens, $\mathrm{VH}$ and retina methanolic and $\mathrm{B} \& \mathrm{D}$ extracts were similar.

\section{Ex-Situ Experimental Optimization:}

The thickness of the 'stamp' affects the sensitivity of the mass spectral data. Because the degree of transfer from the tissue to the MALDI plate is tissue dependent, it is necessary to evaluate the optimal time of contact between the tissue and the plate. If the transfer is not effective, the analytes may be undetectable. Conversely, if the thickness of the stamp is too large, the effectiveness of the ionization suffers.

In addition, the deposition of the matrix onto the stamp is also critical for regional studies. Spreading of matrix can lead to relocation of analytes. In these studies, the spotting of PNA $(0.10 \mathrm{~mL}$ aliquots) on the VH stamps gave the highest sensitivity, followed by the dipping method. For this reason, the same approach was used for the analysis of lens and retina stamps. Overall, both the extraction efficiency and matrix deposition approaches are highly dependent on the nature of the tissue. As expected, the regional analysis of 'stamped' tissue reveals more details on the PL distribution than the in-vitro studies where the results correspond the extracted PLs from the pooled tissue. As a consequence, the sensitivity obtained in the ex-situ mass spectral data is lower than that seen in the in-vitro studies.

\section{PL regional distribution}

In-vitro Studies:

Among the three tissues tested, most PLs were found in the retina followed by the 
lens and then the VH. The VH contained mostly PC(34:1) followed by PC(32:0). Within the $\mathrm{VH}$, the greater abundance of all detected PLs was found in the posterior region followed by the anterior and center zones. No species were detected in the negative mode for the VH. The negative mode showed the lens had $\operatorname{PEp}(36: 2)$ and (34:1) with the highest $\mathrm{S} / \mathrm{N}$. These species have been previously reported to be present in porcine lens. ${ }^{85,147} \operatorname{PEp}(36: 2)$ was also detected in porcine lens using desorption electrospray ionization MS. ${ }^{148}$ The retina showed a great abundance of unsaturated PLs; PEp(38:4), PS(36:1), and PI(38:4). As shown in Table 2-3, high abundance of PI followed by PE and PS species has also been reported in mice, salamander, and human retinas. ${ }^{84,149,150}$ Ex-Situ Studies:

The general trends seen in the in-vitro studies were also followed by the data collected ex situ. Indeed, there was a greater abundance of PLs in the retina relative to the lens and $\mathrm{VH}$ as well as a higher degree of unsaturation PC(34:1), (38:4), and (40:6) in the retina and posterior region of the $\mathrm{VH}$ than in the lens where saturated species are predominant, $\mathrm{PC}(30: 0)$ and $\mathrm{PC}(32: 0)$. The high levels of unsaturated species in retinas from various animals have been reported (see Table $2-3) .{ }^{84,149,150}$ The only variation was to the presence of minute levels of PS(34:1) in the porcine retina reported herein. Unlike human lenses that contain high levels of dihydrosphingomyelin, this sphingolipid was not detected in the porcine lens tissue most likely due to the young age of the porcine tissue. $^{81,142}$

The similarities of the PL species detected in the posterior region of the $\mathrm{VH}$ and the retina suggests that the retina is the source from which the VH lipids are derived. Interestingly, PCs, triacylglycerides, and cholesteryl esters have been reported as the 


\begin{tabular}{|c|c|c|}
\hline Retinal Tissue & Positive Mode & Negative Mode \\
\hline Porcine* (Our data) & $\begin{array}{l}\text { PC(34:1) } \\
\text { PC(38:4) } \\
\text { PC(40:6) } \\
\text { PC(32:0) } \\
\text { SM(16:0) } \\
\text { SM(24:1) }\end{array}$ & $\begin{array}{l}\text { PEp(38:4) } \\
\text { PEp(36:2) } \\
\text { PEp(34:1) } \\
\text { PE(36:1) } \\
\text { PS(36:1) } \\
\text { PI(38:4) }\end{array}$ \\
\hline Human $^{84}$ & $\begin{array}{l}\text { PC }(34: 1) \\
\operatorname{PC}(32: 0) \\
\operatorname{PC}(38: 6) \\
\operatorname{PC}(40: 6) \\
\operatorname{PC}(36: 1) \\
\operatorname{PC}(38: 4) \\
\operatorname{PC}(36: 1)\end{array}$ & $\begin{array}{l}\operatorname{PE}(36: 2) \\
\operatorname{PI}(38: 4) \\
\operatorname{PEp}(36: 2) \\
\operatorname{PEp}(34: 1) \\
\operatorname{PEp}(36: 1) \\
\operatorname{PS}(38: 4) \\
\operatorname{PS}(36: 1) \\
\operatorname{PS}(38: 4)\end{array}$ \\
\hline Mice $^{149,155}$ & $\begin{array}{l}\mathrm{PC}(34: 1) \\
\operatorname{PC}(32: 0) \\
\operatorname{PC}(38: 6) \\
\operatorname{PC}(40: 6)\end{array}$ & $\begin{array}{l}\text { PEp(40:6) } \\
\text { PI(38:4) } \\
\text { PE(44:12) } \\
\text { PS(40:6) }\end{array}$ \\
\hline Salamander ${ }^{150}$ & $\begin{array}{l}\text { PC(32:0) } \\
\text { PC(32:1) } \\
\text { PC(34:1) } \\
\text { PC(36:4) } \\
\text { PC(38:6) } \\
\text { PC(40:6) }\end{array}$ & \\
\hline
\end{tabular}

\begin{tabular}{|c|c|c|}
\hline Lens Tissue & Positive Mode & Negative Mode \\
\hline Porcine* (Our Data) & $\begin{array}{l}\text { SM(16:0) } \\
\operatorname{PC}(30: 0) \\
\operatorname{PC}(32: 0) \\
\operatorname{PC}(34: 1)\end{array}$ & $\begin{array}{l}\operatorname{PEp}(36: 2) \\
\operatorname{PEp}(34: 1) \\
\operatorname{PEp}(38: 4) \\
\operatorname{PS}(34: 1) \\
\text { PI(36:2) }\end{array}$ \\
\hline Porcine $e^{85,90,122}$ & $\begin{array}{l}\mathrm{PC}(32: 0) \\
\mathrm{PC}(34: 1) \\
\mathrm{PC}(30: 0) \\
\mathrm{SM}(16: 0)\end{array}$ & $\begin{array}{l}\operatorname{PEp}(36: 2) \\
\operatorname{PEp}(34: 1) \\
\operatorname{PEp}(36: 1) \\
\operatorname{PE}(36: 2)\end{array}$ \\
\hline Human $^{148}$ & $\begin{array}{l}\operatorname{DHSM}(16: 0) \\
\operatorname{DHSM}(24: 1)\end{array}$ & $\operatorname{PE}(36: 2)$ \\
\hline
\end{tabular}

Table 2-3: A summary of the lipids found in the retina and lens of various mammals. The data listed as Porcine* is the data collected from the current study. The rest of the data come from other papers. 
main components in human and porcine blood plasma with trace amounts of SM, LPC, and PI identified in other mammalian plasma. ${ }^{151-153}$ Porcine blood plasma contains about $1000 \mathrm{nmol} / \mathrm{g}$ TAGs, $1800 \mathrm{nmol} / \mathrm{g}$ PCs, and $1500 \mathrm{nmol} / \mathrm{g}$ cholesteryl esters. ${ }^{154}$ It is therefore possible that the abundance of PCs observed in the posterior $\mathrm{VH}$ could also be a consequence of plasma leakage from the retina vasculature. Furthermore, the high degree of unsaturation in the PLs detected in the posterior VH makes their acyl chains very pliable and thus able to intercalate within the collagen-hyaluronan-water network. As a result, the intermolecular forces holding the network together are proposed to be disrupted and lead to liquefaction. Future studies will address the age-dependence of these molecular changes.

\section{CONCLUSIONS}

PLs have previously been reported in the VH but the regional compositional differences were not known. Since there is no lipid synthesis in VH, the species observed are derived from adjacent tissues. ${ }^{59}$ The results of this work suggest that the PLs present in the posterior $\mathrm{VH}$ migrate from the retina and associated vasculature and those in the anterior $\mathrm{VH}$ from the lens. Since the saturated PLs are more abundant in the lens, it is proposed that they are able to migrate from the lens to the vitreous. This is supported by the presence of saturated species in the anterior region. Molecules, such as fatty acids, and fluids have been reported to migrate into the VH from the lens. ${ }^{156,157}$ However, not all species found in the lens have been observed in the VH. For example, whereas cholesterol is present in lens extracts, it was not detected in the $\mathrm{VH}$.

Conversely, there is a higher relative abundance of unsaturated species in the 
posterior $\mathrm{VH}$ and the retina. It is probable that the retina and associated vasculature could contribute to the unsaturated species found in the posterior VH. Previous studies indicate that the retina impacts the biochemistry of the $\mathrm{VH} .{ }^{158}$ Liquefaction begins in the posterior region of the VH. Since there are more unsaturated PLs in the posterior, they could play a role in liquefaction. Our studies suggest that the permanent positive charge of the headgroup of PCs and SMs allows for stronger interactions with the anionic hyaluronan, relative to other PL headgroups. Furthermore, the greater degree of unsaturation of PCs detected in the retina and posterior $\mathrm{VH}$ suggests that the greater flexibility of the unsaturated acyl chains could contribute to their intercalation within the water-collagenhyaluronan network thus leading to disruption of the molecular ensemble and potential liquefaction of the $\mathrm{VH}$. 


\section{CHAPTER 3 \\ IN-VITRO AND EX-SITU REGIONAL MASS SPECTRAL ANALYSIS OF \\ PHOSPHOLIPIDS AND GLUCOSE IN THE VITREOUS HUMOR FROM DIABETIC AND NON-DIABETIC HUMAN DONORS}

\section{INTRODUCTION}

Diabetes occurs when the blood has a high level of glucose over an extended time. The elevated glucose levels can cause serious health problems to the entire human body including ocular diseases such as macular degeneration, retinopathy, glaucoma, and cataracts. The effect of diabetes on the lens and retina has been previously reported. The lens has a higher abundance of sugars in diabetics compared to non-diabetics. ${ }^{45}$ Diabetes also causes thickening of the lens, changes in the refractive index, and increased membrane permeability. ${ }^{159}$ The consequences diabetes has on the retina stem from the thinning and breakdown of the BRB. The BRB is broken down from the vascular growth factor and induces retinal leukostasis that directly leads to diabetic retinopathy. ${ }^{160}$ Diabetes also causes the inner retina layer to become thinner and causes photoreceptor loss. ${ }^{161}$ Another ocular component affected by diabetes is the VH.

The VH occupies about $80 \%$ of the ocular volume and is located between the lens and the retina. It is made up of about $99 \%$ water and despite this large water content, it has a gelatinous consistency resulting from the hydration of a collagen-HA network. Other components found at minute concentrations in the VH include chondroitin sulfate proteoglycans, versican, proteins, PLs, glucose, and lactate..$^{1,38}$ 
During adolescence the $\mathrm{VH}$ begins to liquefy. This process starts earlier in diabetics. Children with type 1 diabetes have vitreal liquefaction at levels comparable to those in middle-aged healthy adults. ${ }^{162}$ The molecular structural changes that lead to disruption of the water-collagen-HA network are not well understood.

A powerful analytical method that has been used to study ocular tissues is matrix assisted laser desorption ionization mass spectroscopy (MALDI/MS). MALDI/MS has been applied to determine the regional composition of lenticular and retinal tissues. Indeed, with the application of MALDI/MS, the phospholipid (PL) composition in the lens shows more saturated species where the retinal tissue contains more unsaturated PL species. $^{84,85,90,122,148-150,155}$

The very high content of water in the VH makes it challenging to detect components in very low concentrations. Even with the limitations presented by the tissue, it has been studied. The VH regional composition has been explored using MALDI/MS on porcine eyes and showed that the highest abundance of PLs are in the posterior region followed by the anterior and central regions of the VH. ${ }^{163}$ The PL regional distribution in human VHs and the effect of diabetes on liquefaction have yet to be studied. Liquefaction of the VH can lead to serious ocular diseases. The VH is considered to be a repository tissue for adjacent tissues. Therefore, it is important to know the effect(s) that elevated glucose levels and weakening of the retinal barrier have on the distribution of PLs and glucose in the VH.

\section{EXPERIMENTAL}

\section{Materials}


para-Nitroaniline (PNA), chloroform $\left(\mathrm{CHCl}_{3}\right)$, and methanol $(\mathrm{MeOH})$ were purchased from Aldrich Chemical Co., Inc. (Milwaukee, WI). Human globes (3 normal (63 \pm 13 yo); 3 diabetic type $2(58 \pm 11$ yo)) were acquired from the Kentucky Lions Eye Bank and immediately frozen at $-4 \mathrm{C}$.

\section{In-Vitro analysis}

The $\mathrm{VH}$ of each frozen human globe was removed and sectioned into the anterior (closest to the lens), central, and posterior regions. The outer edges of each section were removed to avoid contamination from the other regions. Each section underwent the Bligh and Dyer extraction using a biphasic solvent system of $\mathrm{CHCl}_{3}: \mathrm{MeOH}: \mathrm{H}_{2} \mathrm{O}$ $(2: 1: 1){ }^{141}$ The organic layer of each extraction was added to PNA $(26 \mathrm{mg} / \mathrm{mL})$ and 1,2 dimyristoyl-sn-glycero-3-phosphocholine (DMPC) $(0.005 \mathrm{mg} / \mathrm{mL})$ was added to each mixture as an internal reference.

\section{Ex-Situ analysis}

Each human globe was cut in half along the sagittal plane. The VH was removed from one half and placed onto a clean stainless steel MALDI plate for 10 seconds and removed. The 'stamped' tissue was allowed to dry completely before analysis.

\section{Matrix application}

The matrix used for the analysis of phospholipids was PNA dissolved in methanol (26 mg PNA/mL methanol). For in-vitro analysis, the extract and the PNA solution were premixed. Several $0.75-\mu \mathrm{L}$ aliquots were spotted onto a stainless steel MALDI plate. For ex-situ studies, small aliquots $(0.1 \mu \mathrm{L})$ of PNA were spotted directly onto the stamped tissue. The small volume of the added matrix avoided spreading. Interestingly, glucose was detected using no matrix and a higher laser intensity of the $\mathrm{VH}$ tissue. 


\section{Data acquisition}

The mass spectrometer MALDI-TOF/MS, Voyager Biospectrometry DE workstation was used. It is equipped with a nitrogen laser that emits at $337 \mathrm{~nm}$. The positive-ion mode was used in the reflector mode. An accelerating voltage of $20000 \mathrm{~V}$ was applied after an extraction delay time of $75 \mathrm{nsec}$. The laser power was adjusted to allow minimum fragmentation of analytes while achieving high sensitivity. The $\mathrm{m} / \mathrm{z}$ range chosen extended from 50 to 1200 with an average of 75 shots per spectrum. Data were processed using Data Explorer.

\section{Statistical Analysis}

Two-way Analysis of Variance (ANOVA) was used to compare the effect of diabetes (Non-Diabetic vs Diabetic) and the location within the VH. $\mathrm{p} \leq 0.05$ was considered statistically significant. Data are reported as mean \pm standard error of mean (SEM) for each group.

\section{RESULTS}

\section{In-Vitro results}

Mass spectral traces

Figure 3-1 shows the mass spectra obtained for the regional extracts of non-diabetic VHs $(\mathrm{VH} n d)$ and diabetic VHs $(V H d)$. The posterior region extract exhibited the highest relative abundance of PLs followed by the extracts from the anterior and the central regions. The reported value for the relative intensity is the intensity of the respective peak relative to the matrix peak, which is the most intense peak on the mass spectrum. The most prominent species detected was PC with 34 carbons and one double bond, PC(34:1) 

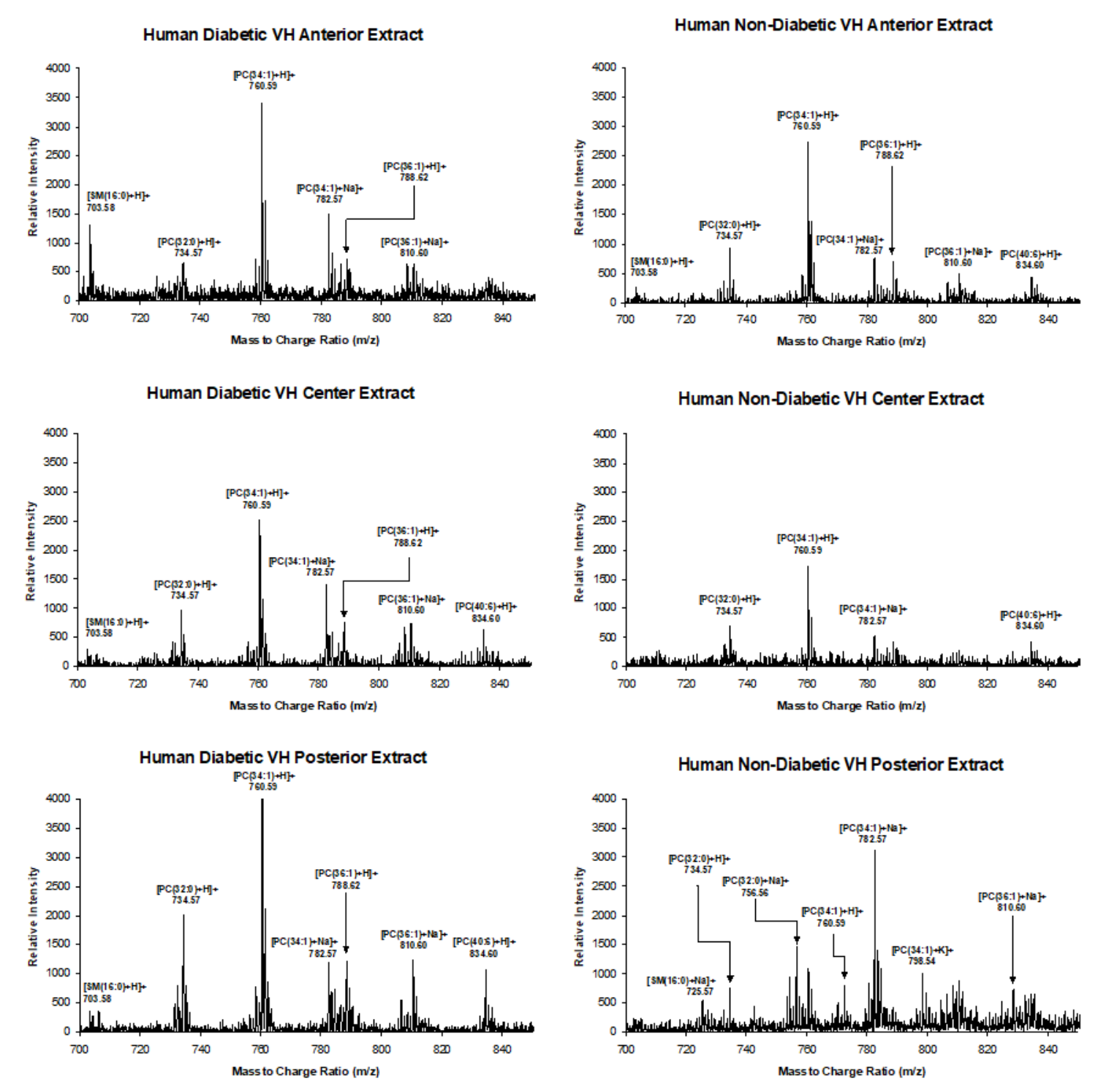

Figure 3-1. MALDI-TOF spectra obtained in-vitro from extracts of $\mathrm{VH} d$ and $\mathrm{VH} n d$. The extracted posterior region shows the highest relative intensity of PLs. VHd extracts have an overall higher relative PL abundance than the extracts from VHnd. 
and its relative intensity before normalization was significantly higher in the posterior regions of $\mathrm{VH} d(12.3 \pm 6.1)$ extracts relative to the $\mathrm{VH} n d(4.0 \pm 2.3)$ ones. The anterior and central regional spectral traces showed more similar relative intensities of $[\mathrm{PC}(34: 1)+\mathrm{H}]^{+}$but the $\mathrm{VH} d(4.2 \pm 2.8,4.0 \pm 3.6)$ were still more abundant than the VHnd $(3.7 \pm 2.1,2.6 \pm 2.1)$. The relative changes of the anterior and central regions compared to the posterior region of $\mathrm{VH} d$ and $\mathrm{VH}$ nd were also explored and showed significant changes ( $\rho=0.0007,2$-way ANOVA; Fig. 3-2). The percent error equation was used to determine the changes in total PL abundance in the anterior and central regions of VHs compared to the posterior region. The $\mathrm{VH} d$ had more variation between the posterior region compared to the anterior and central regions $(0.51 \pm 0.07,0.67 \pm 0.03)$ than VHnd $(0.84 \pm 0.04,0.78 \pm 0.08)$ due to the $\mathrm{VH} d$ having a greater abundance of PLs the posterior than the anterior regions compared to the VHnd. VHnd had comparable levels among the regions, which is why the values comparing the variation were closer to 1. The total relative abundances of PLs after normalization, corresponding to the anterior $(2.3 \pm 0.7)$ and central $(2.1 \pm 0.5)$ regions of $\mathrm{VH} d$ were slightly more abundant to those measured in VHnd $(1.0 \pm 0.7$ and $2.0 \pm 0.7$ respectively) samples. However, the extract of the posterior region of $\mathrm{VH} d$ showed about three times greater PL abundance $(6.4 \pm$ 3.7) than the corresponding VHnd extract $(2.1 \pm 1.3)$.

\section{$P C$ regional distribution}

The relative intensity of the peaks was normalized to the DMPC peak for each spectrum. All regional $\mathrm{VH} d$ extracts showed that $\mathrm{PC}(34: 1)$ is the most abundant species. $\mathrm{PC}(34: 1)$ has the highest abundance in the posterior extract $(14.8 \pm 1.3$ for $\mathrm{VH} d$ and $7.9 \pm 2.9$ for 


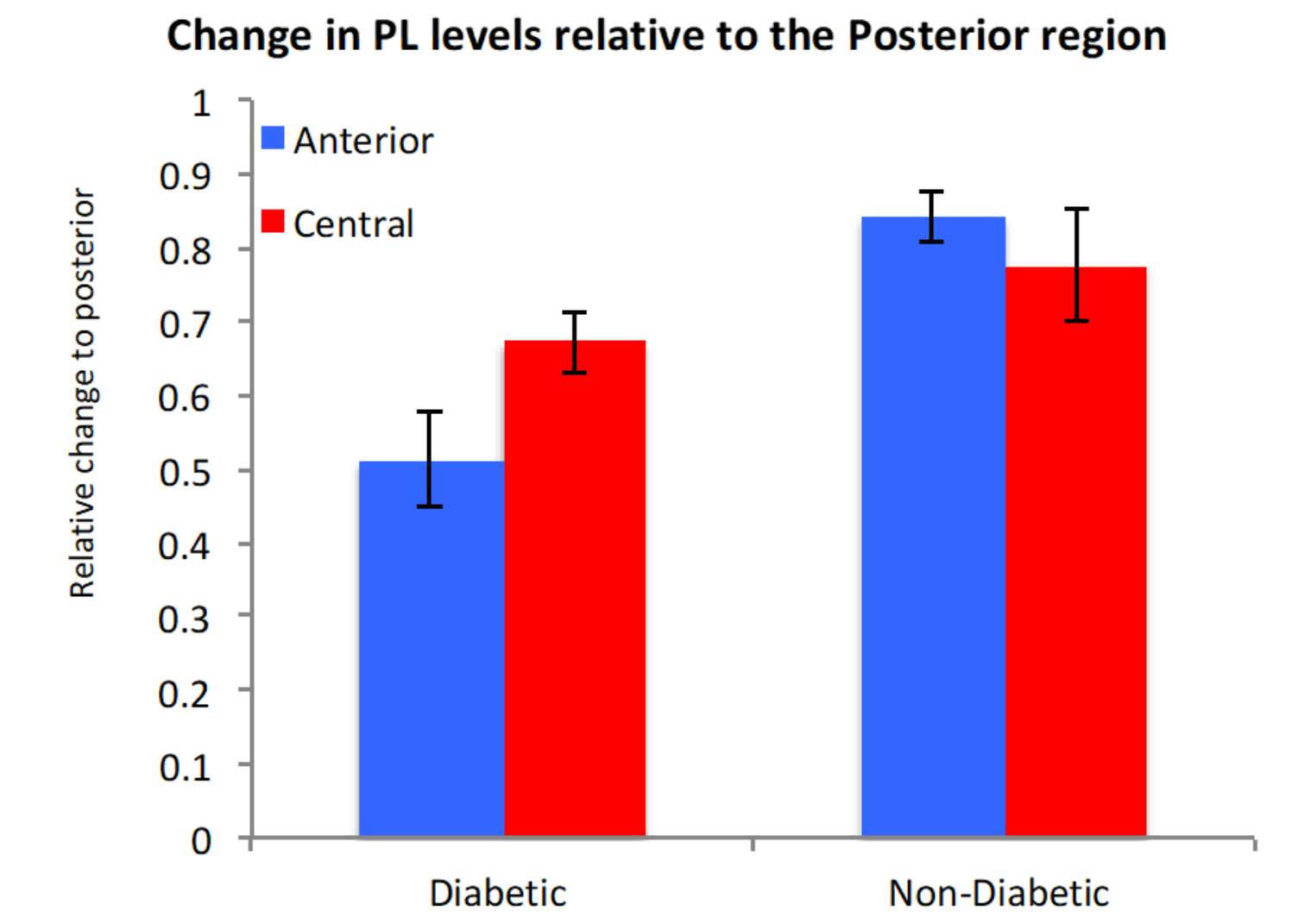

Figure 3-2. Comparison of the total PL composition relative to the posterior region in the regional analysis of VHd and VHnd using in-vitro techniques. The posterior region has the highest abundance of each PL species and is used as a standard to determine the relative change for the anterior and central regions. Data are presented as mean \pm SEM for each eye $(n=6)$. Two-way ANOVA showed there was variation between the VHd and VHnd data sets $(\rho<0.05)$. 
$\mathrm{VH} n d)$, followed by the anterior extract $(10.6 \pm 1.4$ for $\mathrm{VH} d$ and $2.8 \pm 1.1$ for $\mathrm{VH} n d)$, and the central extract $(4.6 \pm 0.7$ for $\mathrm{VH} d$ and $4.6 \pm 2.1$ for $\mathrm{VH} n d)$. The extracts show that $\mathrm{PC}(32: 0)$ and $\mathrm{PC}(36: 1)$ are the second and third most abundant PC species in the regional extracts. The posterior region shows the highest normalized relative intensity for $\mathrm{PC}(32: 0)(11.4 \pm 2.4$ for $\mathrm{VH} d$ and $3.3 \pm 1.3$ for $\mathrm{VH} n d)$. $\mathrm{PC}(32: 0)$ in the anterior $(5.6 \pm$ 0.8 for $\mathrm{VH} d$ and $1.0 \pm 0.4$ for $\mathrm{VH} n d)$ and central regions $(4.3 \pm 0.5$ for $\mathrm{VH} d$ and $1.8 \pm 0.9$ for $\mathrm{VH} n d)$ are more comparable to each other. For PC(36:1), the anterior regions show that the average normalized relative intensity is $3.6 \pm 0.5$ for $\mathrm{VH} d$ and $1.0 \pm 0.3$ for $\mathrm{VH} n d$, the central regions values are $1.8 \pm 0.8$ for $\mathrm{VH} d$ and $1.6 \pm 0.7$ for $\mathrm{VH} n d$, and the posterior region values are $5.1 \pm 0.5$ for $\mathrm{VH} d$ and $3.4 \pm 1.3$ for $\mathrm{VH} n d$.

\section{Sphingomyelin (SM) regional distribution}

The distribution of SM also varied between extracts of $\mathrm{VH} d$ and $\mathrm{VH} n d$. The extract of the anterior region of $\mathrm{VH} d$ had a relative SM intensity of $1.7 \pm 0.2$ and the posterior had 1.6 \pm 0.3 for SM. The corresponding extracts of VHnd showed lower levels of SM but relative abundance of $\mathrm{SM}$ in the posterior region was nearly twice $(1.0 \pm 0.3)$ greater than in the anterior region $(0.45 \pm 0.15)$.

\section{Ex-situ Results}

\section{Mass spectral traces}

Representative mass spectral traces obtained for the 'stamped' regions of $\mathrm{VH} d$ and $\mathrm{VH} n d$ are shown in Figure 3-3. The posterior regions showed the highest relative abundance of PLs, followed by the anterior and then the central regions. The VHd stamp had the 

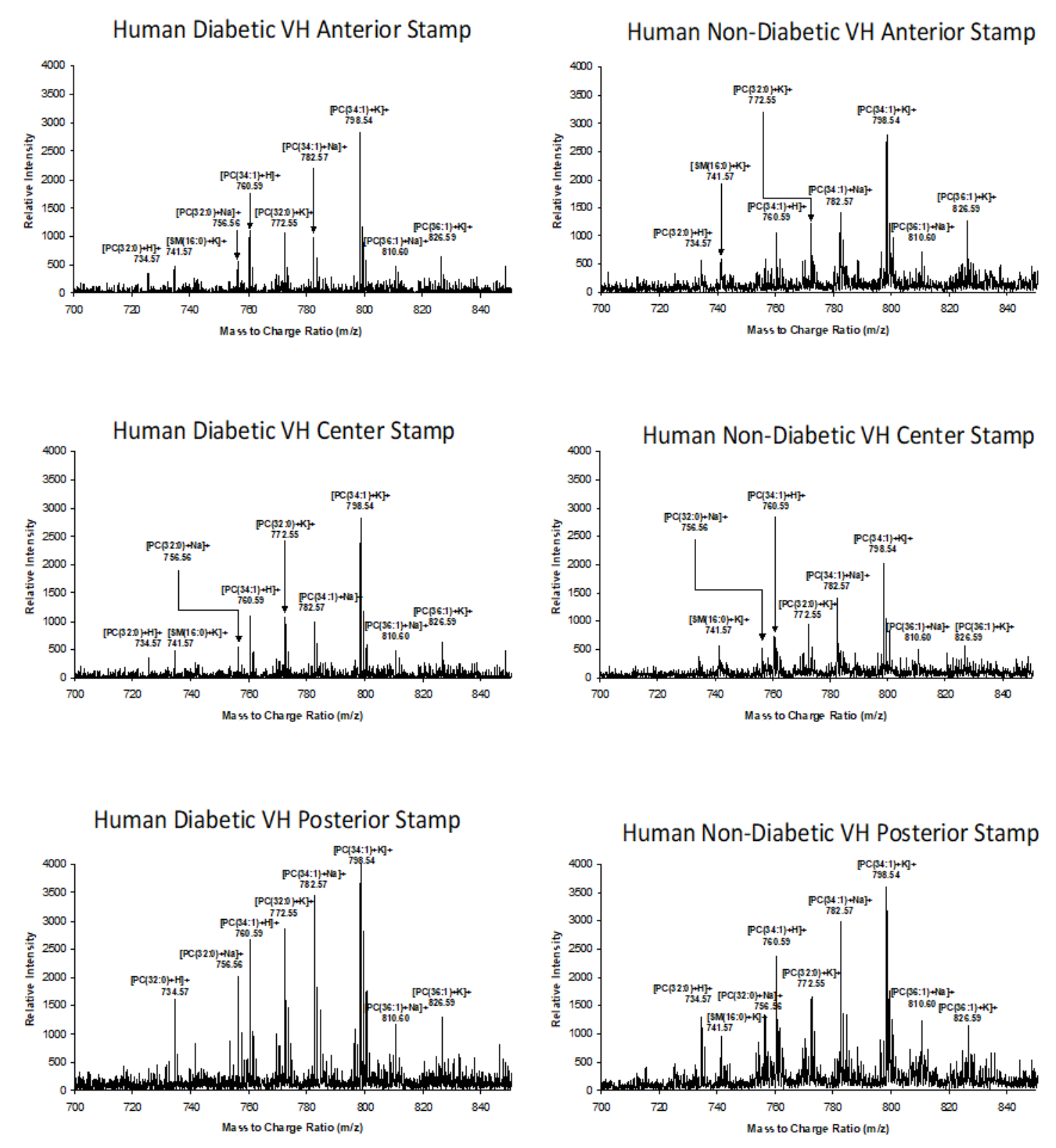

Figure 3-3. MALDI-TOF spectra of stamped VH $d$ and VHnd. The posterior region of the stamps shows the highest relative intensity of PLs. 
highest relative intensity for $[\mathrm{PC}(34: 1)+\mathrm{K}]^{+}$in the posterior $(5.5 \pm 3.7)$ compared to the corresponding regions in $\mathrm{VH} n d$ stamps $(1.5 \pm 0.8)$. The $\mathrm{VH} d$ stamp exhibited comparable $\mathrm{S} / \mathrm{N}$ ratios for $[\mathrm{SM}(16: 0)+\mathrm{K})]^{+}$in the anterior $(1.5 \pm 0.8)$ and the posterior $(1.5 \pm 0.8)$ but these values were significantly ( $\rho=0.005$ ) lower for the $\mathrm{VH} n d$ (posterior: $0.92 \pm 0.23$ and anterior: $0.78 \pm 0.22)$.

PC regional distribution

Figure 3-4 shows the distribution of the total amount of PLs throughout the stamps of $\mathrm{VH} d$ and VHnd. Two-way ANOVA confirmed variations between the location on the stamp and between VHd and VHnd $(\rho=0.02)$. The stamps of VH $d$ showed the highest relative abundance of PLs compared to those of $\mathrm{VH} n d$. The posterior region showed the greatest relative abundance of PCs followed by the anterior and central regions in both $\mathrm{VH} d(32 \pm 4.7,23 \pm 4.9,17 \pm 2.5$, respectively) and $\mathrm{VH} n d .(16 \pm 4.5,11 \pm 2.9,12 \pm 3.1$, respectively). The most prominent species throughout the stamps was $\mathrm{PC}(34: 1)$. The posterior region of $\mathrm{VH} d$ detected $15 \pm 2.3$ relative intensity for $\mathrm{PC}(34: 1)$ compared to 7.7 \pm 1.1 for the anterior region. The VHnd showed less variation in the relative intensity of the posterior region $(9.2 \pm 2.4)$ compared to the anterior region $(6.3 \pm 1.4)$ for $\mathrm{PC}(34: 1)$. $\mathrm{PC}(32: 0)$ was more abundant in the $\mathrm{VH} d(7.1 \pm 1.4$ posterior, $6.6 \pm 1.8$ central, and $4.1 \pm$ 1.5 anterior) compared to the $\mathrm{VH} n d(3.6 \pm 1.1$ posterior, $2.8 \pm 0.8$ central, and $3.0 \pm 1.0$ anterior). This trend was followed with $\mathrm{PC}(36: 1)$ in the $\mathrm{VH} d(4.9 \pm 0.7$ posterior, $3.6 \pm$ 0.7 central, and $2.7 \pm 0.4$ anterior) and $\mathrm{VH} n d(3.0 \pm 0.9$ posterior, $2.2 \pm 0.5$ central, and $2.2 \pm 0.7$ anterior) as well.

SM regional distribution 


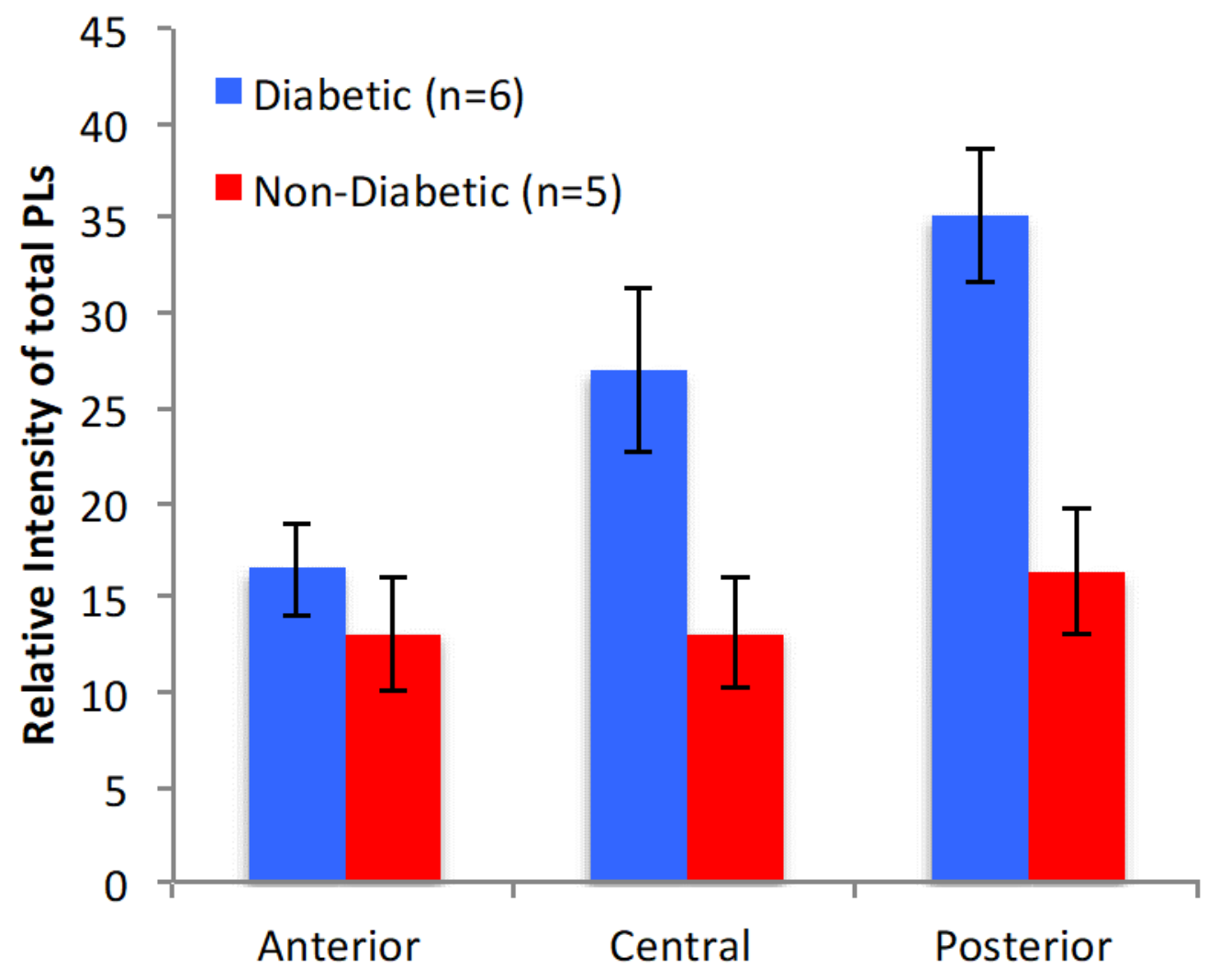

Figure 3-4. Comparison of the PL composition in the regional ex-situ analysis of $\mathrm{VH} d$ and VHnd stamps. The posterior region has the highest abundance of total PL species, followed by the anterior and central regions. Data are presented as mean \pm SEM for each group. Two-way ANOVA showed there was variation between the VHd and VHnd as well as the location on the stamps $(\rho<0.05)$. 
For VHd stamps, higher abundance of SM was observed in the posterior $(2.5 \pm 0.4)$ compared to the anterior $(1.9 \pm 0.2)$ region. The opposite trend was seen for $\mathrm{VH} n d$ stamps for which the anterior regions had a higher relative abundance of SM $(2.6 \pm 1.3)$ compared to the posterior regions $(1.9 \pm 0.5)$.

\section{Glucose regional distribution}

The analysis of glucose did not require the use of a matrix because HA acted as a matrix and was able to absorb the $337 \mathrm{~nm}$ wavelength of the laser and ionize glucose. Stamps of both $\mathrm{VH} d$ and $\mathrm{VH} n d$ were analyzed without matrix to determine glucose levels and showed a variation between the two groups ( $\rho=0.003$, 2-way ANOVA; Fig. 3-5). Higher relative abundance of glucose was observed for $\mathrm{VH} d$ than $\mathrm{VH} n d$. The posterior region of VH $d$ stamps had a significantly higher level of glucose $(12 \pm 1.3)$ compared to the central $(6.7 \pm 0.8)$ and anterior $(8.1 \pm 1.0)$ regions. The VHnd stamps showed more comparable levels of glucose throughout the three regions (posterior: $6.5 \pm 0.7$, central: $5.9 \pm 1.2$ and anterior: $4.7 \pm 0.9$ ).

\section{DISCUSSION}

For this investigation we chose a soft form of ionization, MALDI, not only to minimize analyte fragmentation but also to enable the direct analysis of ionizable species without significant sample manipulation. Although the presence of PLs was reported

previously, their regional distribution was not known. ${ }^{164}$ Similarly, glucose has been detected in the $\mathrm{VH}$ but its regional variations were not known in human tissue. The $\mathrm{VH}$ acts as a repository of metabolites that migrate from adjacent organs. ${ }^{59}$ The ex-situ 


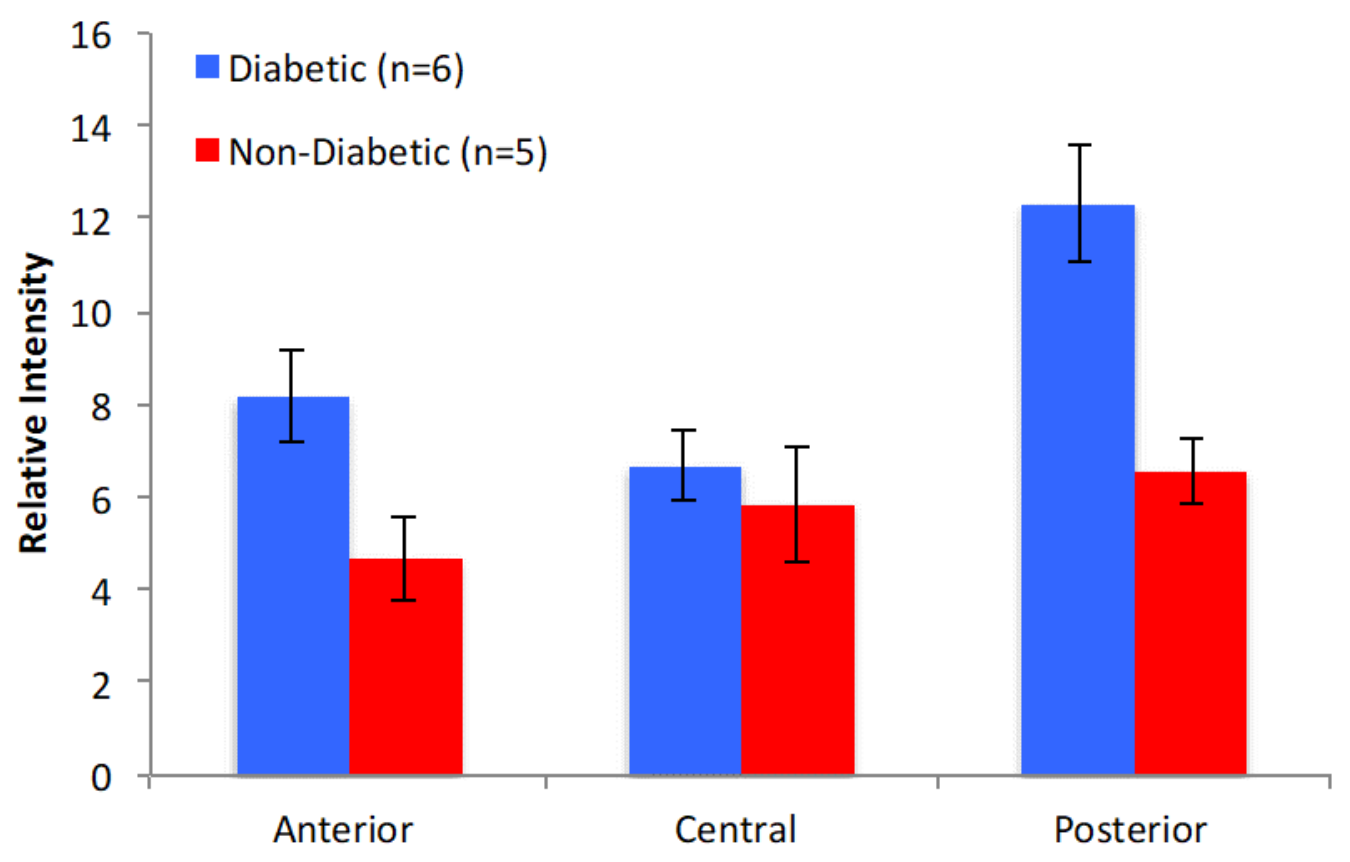

Figure 3-5. Relative intensities of glucose in regions of diabetic VH tissue compared to non-diabetic tissue. In both diabetic and non-diabetic the higher glucose levels were found in the posterior region. Compared to non-diabetic VHs, diabetic VHs had a significantly greater abundance of glucose throughout the tissue. Data are presented as mean \pm SEM for each group. Two-way ANOVA showed there were variations between the VHd and VHnd stamps as well as the location on the stamp for the abundance of glucose $(\rho<0.05)$. 
analysis and in-vitro analysis showed similar trends with the distribution of PLs. The posterior region contained the most PLs in both $\mathrm{VH} d$ and $\mathrm{VH} n d$. The most abundant species was PC(34:1) and the levels of this PL were also found to be present in the highest amount in VHs from porcine with previous data found in porcine eyes VHs. ${ }^{163}$

The sensitivity and precision of the in-vitro analyses of human VHs were higher than those for ex-situ studies. This is the result of both, higher levels of PLs in the extract as well as the use of an internal calibrant. Therefore, the quantitative trends obtained invitro are more reliable. The ex-situ analysis was more qualitative but showed regional changes similar to those observed in-vitro. Results from this method indicated that there was a higher percentage of saturated PC species in the anterior than in the posterior regions of both $\mathrm{VH} d$ and $\mathrm{VH} n d$. Previous studies have shown that species from the lens migrate into the VH. ${ }^{156,157}$ Since the lens has more saturated than unsaturated PLs, the saturated PCs could be related to lenticular PLs. However, dihydro-sphingomyelin (DHSM), the most abundant PL ( 55\%) found in human lens membranes, was not detectable in the VH extracts. ${ }^{165}$ This suggests that the migration of PLs from the lens into the $\mathrm{VH}$ is significant lower relative to that from the retina. Furthermore, the strong H-bonding interactions among the interfacial and headgroup regions of DHSM are expected to keep this PL in the lens membranes.

The age of diabetics $(58 \pm 11)$ and non-diabetics $(63 \pm 13)$ were similar so the observed trends are related to diabetes rather than age-related changes. The most noticeable compositional difference between $\mathrm{VH} d$ and $\mathrm{VH} n d$ was the greater abundance of PLs and glucose in $\mathrm{VH} d$. This finding can be related to the weakening of the retinal 
barrier with diabetes. As a result, greater levels of these compounds can migrate from the retina into the $\mathrm{VH}$. In addition, the rate of migration is expected to be higher.

When the $\mathrm{VH}$ liquefies, the $\mathrm{HA} /$ collagen $/ \mathrm{H}_{2} \mathrm{O}$ network collapses. This process is exacerbated by diabetes. ${ }^{162}$ Patients with diabetes have a weaker BRB, which allows molecules to flow more freely into the retina. Since the $\mathrm{VH}$ is a repository for surrounding tissues, the more permeable barrier can contribute to a higher level of glucose and PLs found in the VH along with other metabolites. Diabetes has been shown to increase passive glucose transport into the VH using fluorescein. ${ }^{67}$ Changes in the barrier between the inner retina and VH could lead to the increased permeability ${ }^{67}$

We propose that the greater contents of PLs and glucose in the posterior VH causes the breakage of the strong $\mathrm{H}$-bonding network that gives the $\mathrm{VH}$ its gelatinous consistency. Since HA consists of a spiral-like chain of hydrophobic and hydrophilic patches, the acyl chains of PLs could interact with the hydrophobic side of twirl while the PL headgroups form ionic or H-bonds of HA and 'break apart' the VH arrangement. Similarly, glucose could interact with the HA via H-bonding. Since there are higher amounts of PLs and glucose in the VH of diabetics than non-diabetics, it is proposed that these compounds speed up the collapse of the network leading to the acceleration of liquefaction.

Model studies are underway to determine the interactions between PLs, HA, and glucose to improve our understanding of liquefaction at the molecular and structural levels. 


\section{CHAPTER 4}

\section{INTERACTIONS OF HYALURONAN WITH GLUCOSE AND PHOSPHOLIPIDS DETERMINED USING MALDI/MS AND NMR SPECTROSCOPY}

\section{INTRODUCTION}

Our group has reported regional changes in the levels of phospholipids (PLs) in vitreous humors (VHs) from porcine and human eyes. In addition, significant increases in glucose contents were observed in VHs from non-diabetics and diabetics. The higher levels of both PLs and glucose detected in the posterior part of the VH led us to propose that the intercalation of these compounds within the strong hydrogen-bonded network of water ( $99 \%)$, hyaluronan (HA) and collagen contribute to the liquefaction of the gelatinous VH.

The current works focuses on model studies designed to learn the molecular interactions that take place when PLs and/or glucose are added to hydrated HA. HA is a type of glycosaminoglycan (GAG) made up of repeating units of a disaccharide composed of D-glucuronic acid (U) and N-acetyl-D-glucosamine (A), linked via alternating $\beta-(1 \rightarrow 4)$ and $\beta-(1 \rightarrow 3)$ glycosidic bonds. HA is synthesized in the endoplasmic reticulum and forms long unbranched chains that at concentrations as low as $1 \mathrm{mg} / \mathrm{mL}$ HA becomeentanged. ${ }^{10,3,16}$ This leads to interactions that cause the twisting of the HA chains and causes formation of alternating hydrophilic and hydrophobic 
patches. ${ }^{10}$ The non-ideal osmotic pressure of HA contributes to water homeostasis in the VH. Due to the size and entanglement of the HA chains, diffusion processes are slowed down. ${ }^{16}$

Whereas the interactions of type II collagen and HA have been explored and shown to be minimal, hydrophobic interactions are believed to occur between the terminal region of the collagen and HA but the specific sites involved in these associations remain to be determined. ${ }^{17,19} \mathrm{HA}$ can also interact with proteoglycans, cell membranes, and other receptors such as CD-44. ${ }^{19}$ The interactions between PLs and HA have also been studied using rotary shadowing electron microscopy, gel permeation chromatography, multi-angle laser-light-scattering photometry and nuclear magnetic resonance (NMR) spectroscopy. HA could affect the organization of PLs as they aggregate around HA. ${ }^{15}$ PLs bind to the hydrophobic patches of HA strands that can reduce the HA stiffness. ${ }^{14}$ The specific details of these molecular interactions need to be further explored.

The molecular interactions between glucose and other vitreal components have not been reported previously. In this study, we have investigated the interactions of glucose with HA in the presence and absence of PLs in continuation of a project focusing on the monomeric HA unit and the effect on RIE. ${ }^{166}$

One of the analytical methods used to investigate these possible interaction is matrix-assisted laser desorption ionization (MALDI) mass spectrometry (MS). Although MALDI/MS is a sensitive tool for qualitative and quantitative analysis, it has not been applied, to our knowledge, to reveal intra- and intermolecular interactions. MALDI is a soft form of ionization that involves the use of a matrix (in high concentration) that co- 
crystallizes with the analyte(s). When a laser pulse reaches the sample, the matrix absorbs the radiation and transfers part of it to analytes that are then desorbed and ionized via adduct-formation or electron transfer. These ions enter the mass analyzer and are separated based on their mass-to-charge $(\mathrm{m} / \mathrm{z})$ ratio. ${ }^{118}$ Relevant to our application is the fact that different analytes present in the sample with the same concentration do not produce peaks of comparable intensity in the MS spectrum. ${ }^{167}$ The intensity of each analyte signal depends on its relative ionization efficiency (RIE). Importantly, RIEs are not only affected by the chemical make up of the analyte but also the interactions between the matrix and analyte and other compounds in the sample as they co-crystallize. The activation energies achievable at the temperatures reached in the ion plume, as well as possible collisions once in the ion plume also affect the RIEs. ${ }^{115}$ The RIEs also depend on the environment surrounding the analyte. For example, different types of tissue can lead to changes in RIEs for a specific PL. ${ }^{168}$ For these reasons, this work explores the changes in RIE for PLs and Glu in the presence of HA, in both its polymeric and ultrasonicated forms.

The interactions among these molecules were also studied in solution phase using NMR spectroscopy to help us understand the interactions that take place in the liquid phase. If these interactions remain as the solvent evaporates, they are expected to strengthen. Therefore, it is possible that the RIEs measured via MALDI/MS be affected. In this work, we have measured changes in RIEs when two or more of the compounds mentioned above are present. The findings from these model studies have been tested on actual VHs obtained from porcine eyes to learn the potential molecular/structural changes that take place during vitreous liquefaction. 


\section{EXPERIMENTAL}

\section{Chemicals}

para-Nitroaniline (PNA), 2,5-dihydroxybenzoic acid (DHB), $\alpha$-D-glucose, hyaluronic acid sodium salt from Streptococcus equi (HA) (mol wt $\sim 1.5-1.8$ x $10^{6} \mathrm{Da}$ ), L- $\alpha-$ phosphatidylcholine $(\mathrm{PC})$ from egg yolk, acetonitrile $(\mathrm{ACN})$, and methanol $(\mathrm{MeOH})$ were purchased from Aldrich Chemical Co., Inc. (Milwaukee, WI). Porcine eyes were collected from a local abattoir and immediately dissected or frozen at $-4 \mathrm{C}$.

\section{Matrix Preparation}

The matrices used were 2,5-dihydroxybenzoic acid (DHB) dissolved in water:acetonitrile (1:1) with $1 \%$ trifluoroacetic acid $\left(20 \mathrm{mg} \mathrm{DHB} / \mathrm{mL} \mathrm{H}_{2} \mathrm{O}: \mathrm{ACN}\right)$ and para-nitroanaline (PNA) dissolved in methanol (26 mg PNA/mL methanol).

\section{Model Studies}

Solutions of glucose and HA were prepared in deionized $\mathrm{H}_{2} \mathrm{O}$ for MALDI/MS studies and $\mathrm{D}_{2} \mathrm{O}$ for NMR studies. Two solutions of the HA polymer were prepared by dissolving $5 \mathrm{mg}$ of $\mathrm{HA}$ in $1 \mathrm{~mL}$ of $\mathrm{H}_{2} \mathrm{O}$. One of these solutions was ultrasonicated for 10 minutes with a 2-second pulse $(20 \mathrm{~V})$ using a Sonics and Materials, Danbury, CT, ultrasonicator. PC was dissolved in methanol for MALDI/MS studies and MeOD for NMR studies. The final concentrations were $5 \mathrm{mg} / \mathrm{mL}$ for each component of the mixtures. The RIE values were calculated by calculating the ratio of the sum of the relative intensitiesfor all the signals corresponding to ions of a given analyte versus the sum of the relative intensities of all matrix-related peaks.

\section{Porcine Vitreous Humor Analysis}


A frozen porcine $\mathrm{VH}$ was removed from the eye and cut in half along the sagittal plane. One half was 'stamped' on a clean stainless steel MALDI plate by placing it on the plate and leaving it for $10 \mathrm{~s}$. After removing it, the tissue was ultrasonicated for 10 minutes. About $0.5 \mathrm{~g}$ of the ultrasonicated $\mathrm{VH}$ was removed with a spatula and spread very thinly onto a second MALDI plate. A $0.2-\mu \mathrm{L}$ aliquot of the matrix (either PNA or DHB) was applied on top of the tissue. The second half was extracted using the Bligh and Dyer method ${ }^{141}$ by adding $3.75 \mathrm{~mL}$ of $\mathrm{MeOH}: \mathrm{CHCl}_{3}$ (2:1) per gram of tissue. After sonication, $1.25 \mathrm{~mL}$ of $\mathrm{CHCl}_{3}$ and $1.25 \mathrm{~mL}$ of $\mathrm{H}_{2} \mathrm{O}$ were added.

1,2-dimyristoyl-sn-glycero-3 phosphocholine (DMPC) $(0.005 \mathrm{mg} / \mathrm{mL})$ was added to the organic layer of the extract as an internal calibrant. PNA or DHB was premixed with the analyte, spotted directly on a MALDI plate, and analyzed.

\section{Data Acquisition}

\section{MALDI-TOF/MS Studies}

A matrix-assisted laser desorption ionization time of flight/mass spectrometer (MALDI-TOF/MS, Voyager Biospectrometry DE workstation, Applied Biosystems, Foster City, CA) with a nitrogen laser that emits at $337 \mathrm{~nm}$ was used to analyze all samples. Positive and negative-ion spectra were collected in the reflector mode. An accelerating voltage of $20 \mathrm{kV}$ was applied after an extraction delay time of $75 \mathrm{nsec}$. The laser energy was $70 \pm 5 \mu \mathrm{J}$ and the pulse rate $3 \mathrm{~Hz}$. The laser intensity was adjusted to minimize fragmentation and produce high sensitivity. A mass range of $50-1200 \mathrm{~m} / \mathrm{z}$ was used and 50 shots were averaged per spectrum in both positive and negative mode. Data Explorer software version 4.8 (Applied Biosystems, Foster City, CA) was used to analyze the data. The relative intensities of matrix-related peaks were added and compared to the 
sum of intensities observed for all analyte-related peaks. The ratio of the values was calculated to determine the relative ionization efficiency (RIE) of the analyte.

\section{NMR Studies}

${ }^{1} \mathrm{H}$ NMR spectra were obtained using a Varian VNMRS $700 \mathrm{MHz}$ NMR spectrometer (Palo Alto, CA). Spectra were acquired with 250 scans, $45^{\circ}$ pulse width, and a relaxation delay of 1.000 seconds at $25^{\circ} \mathrm{C}$. The $\mathrm{D}_{2} \mathrm{O}$ resonance was used as reference for the correct determination of chemical shifts.

\section{Statistical Analysis}

One-way Analysis of Variance (ANOVA) was used to compare the effect of HA on the RIE of the analyte. $\mathrm{p} \leq 0.05$ was considered statistically significant. Data are reported as mean \pm one standard deviation for each group.

\section{RESULTS}

In this section we describe first the results obtained by MALDI-TOF/MS and NMR when HA was mixed with PLs. The second portion of this section covers the results obtained when glucose was added to HA alone and HA with PLs. Some of the HA solutions were ultrasonicated to cause fragmentation of the HA polymer and see the effects of disrupting the HA represent conditions similar to those that occur during liquefaction of the $\mathrm{VH}^{169}$ Finally, the results obtained using the vitreous humor from porcine eyes are presented.

\section{Changes in RIE for PLs in the presence of HA and Glucose}

Solutions of PC and PC with HA were premixed with the DHB matrix and analyzed using MALDI-TOF/MS. The HA polymer could not be detected in the mass spectral range used to observe PLs. HA fragments were not observed either. On the other hand, 
PC ionized easily and was detected before and after premixing it with HA (Fig. 4-1). When other PLs and glucose were also mixed with PC, their RIEs were slightly decreased (see Fig. 4-2). The most notable differences in the RIE of PC were seen in the presence of HA ( $\rho=0.025$, 1-way ANOVA). When polymeric HA and PC were premixed, the relative ionization efficiency (RIE) of PC was reduced. The decrease was greater when ultrasonicated HA was mixed with PC. These findings suggest there are interactions between PC and HA in the liquid phase that remain after solvent evaporation. Furthermore, the level of these interactions is increased when ultrasonicated HA is present. The RIE values decreased from $0.40 \pm 0.19$ for PC alone to $0.26 \pm 0.12$ in the presence of $\mathrm{HA}$ and to $0.13 \pm 0.06$ when mixed with ultrasonicated HA. This decreasing trend in the RIE of PC was also reflected in the reduction of the S/N for PC in the mass spectra. The $\mathrm{S} / \mathrm{N}$ of $\mathrm{PC}(34: 1)$ was $(4.7 \pm 2.4) \times 10^{3}$ for PC alone but decreased to $(1.4 \pm$ 0.6) $x 10^{3}$ when HA was added. In the presence of Glu, the $\mathrm{S} / \mathrm{N}$ of $\mathrm{PC}(34: 1)$ was $(2.6 \pm$ 1.0) $\times 10^{3}$.

\section{Changes in NMR spectral features for PLs in the presence of HA and Glucose}

PC was mixed with HA and NMR spectra were acquired as seen in Figure 4-3. When compared to HA alone, the chemical shifts of every HA resonance in the mixture decreased (shifted upfield) indicating that the HA strands were more shielded in the presence of PC. In addition, the resonances corresponding to protons in the acyl chains of PC were no longer detectable in the mixture due to significant broadening. The spectral 
a)

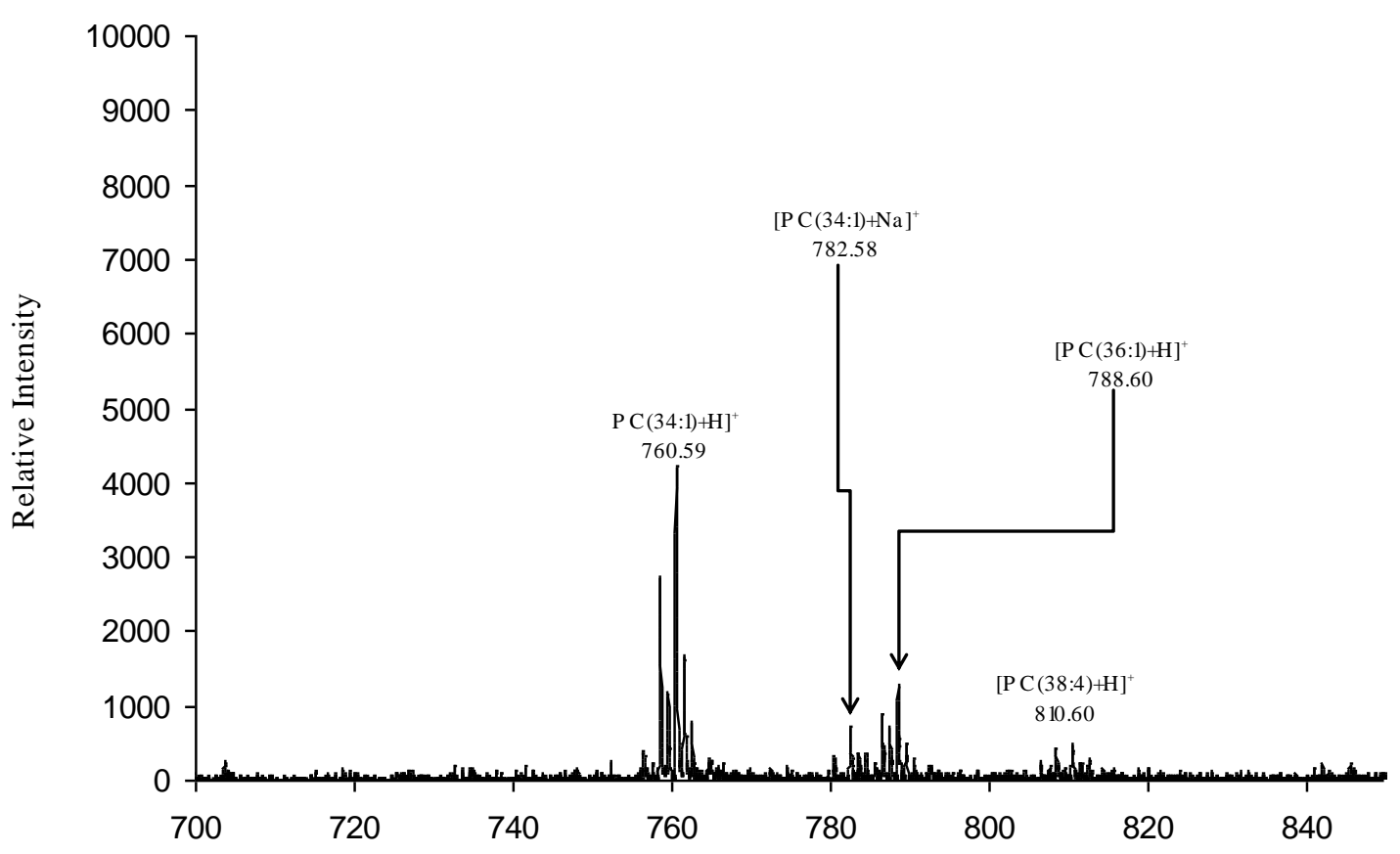

b)

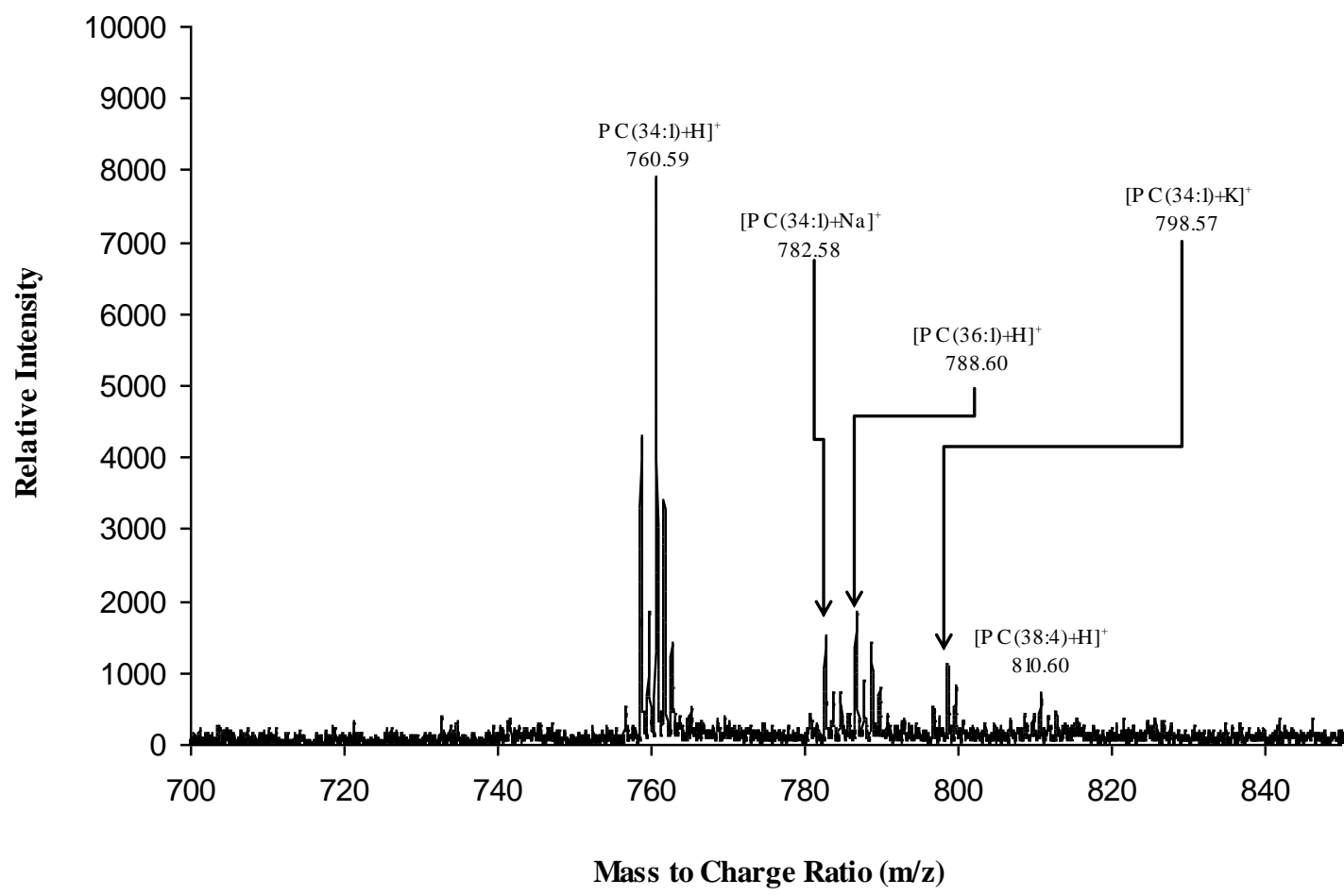

Figure 4-1. The MALDI spectra of a) PC and b) PC with HA. The relative intensities of the PC species decrease in the presence of HA. 


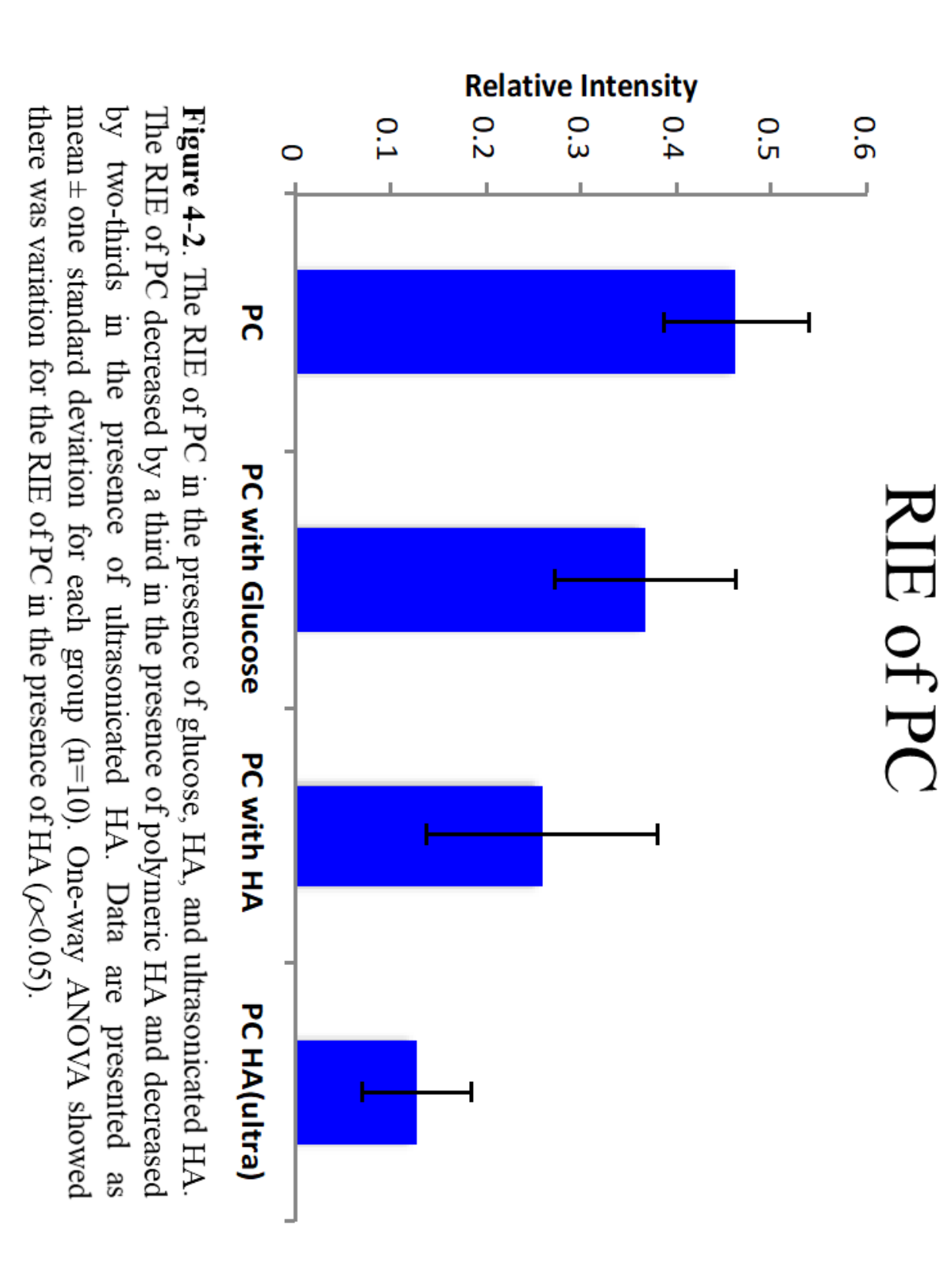


a)

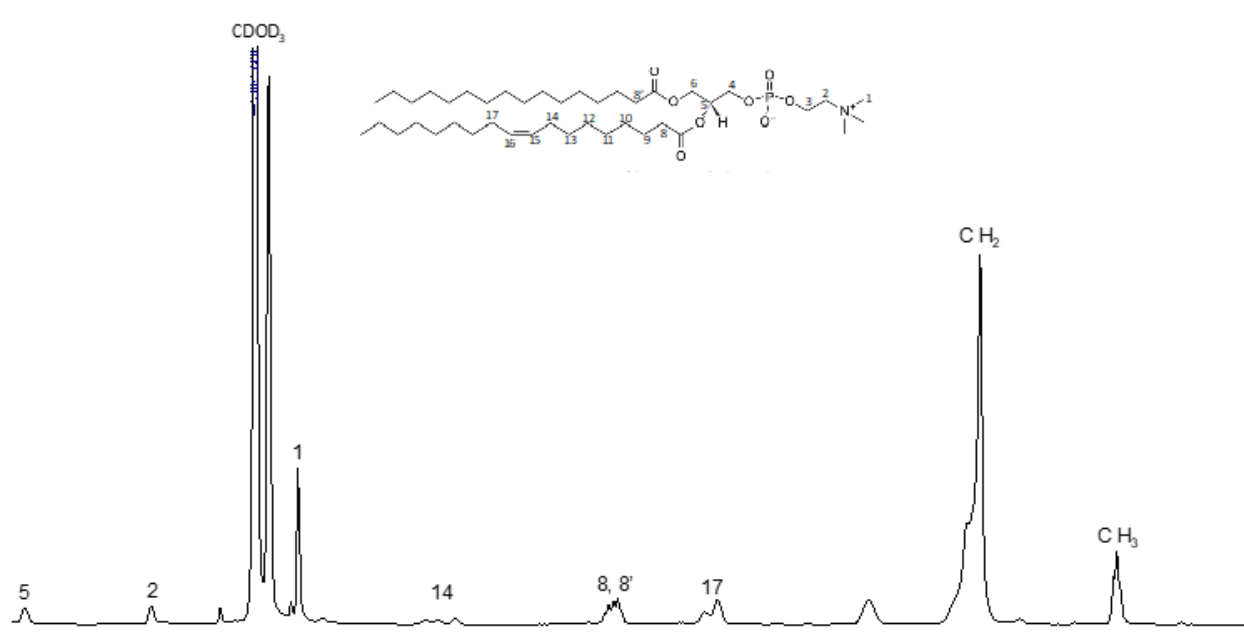

b)
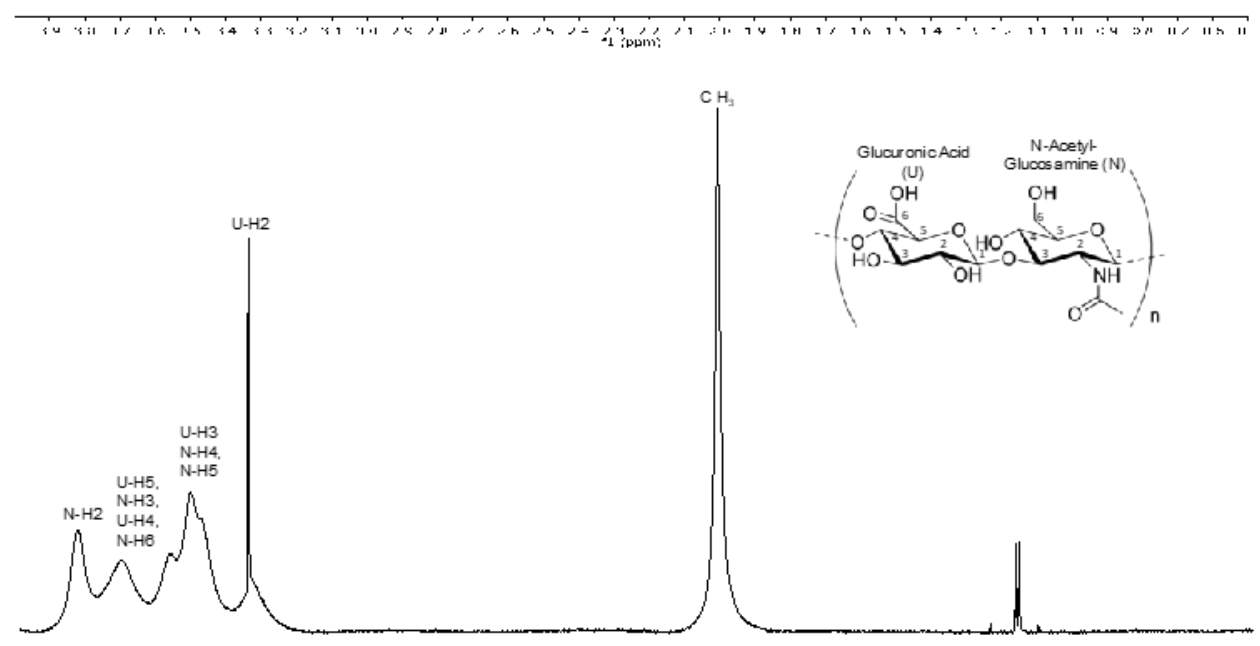

c)

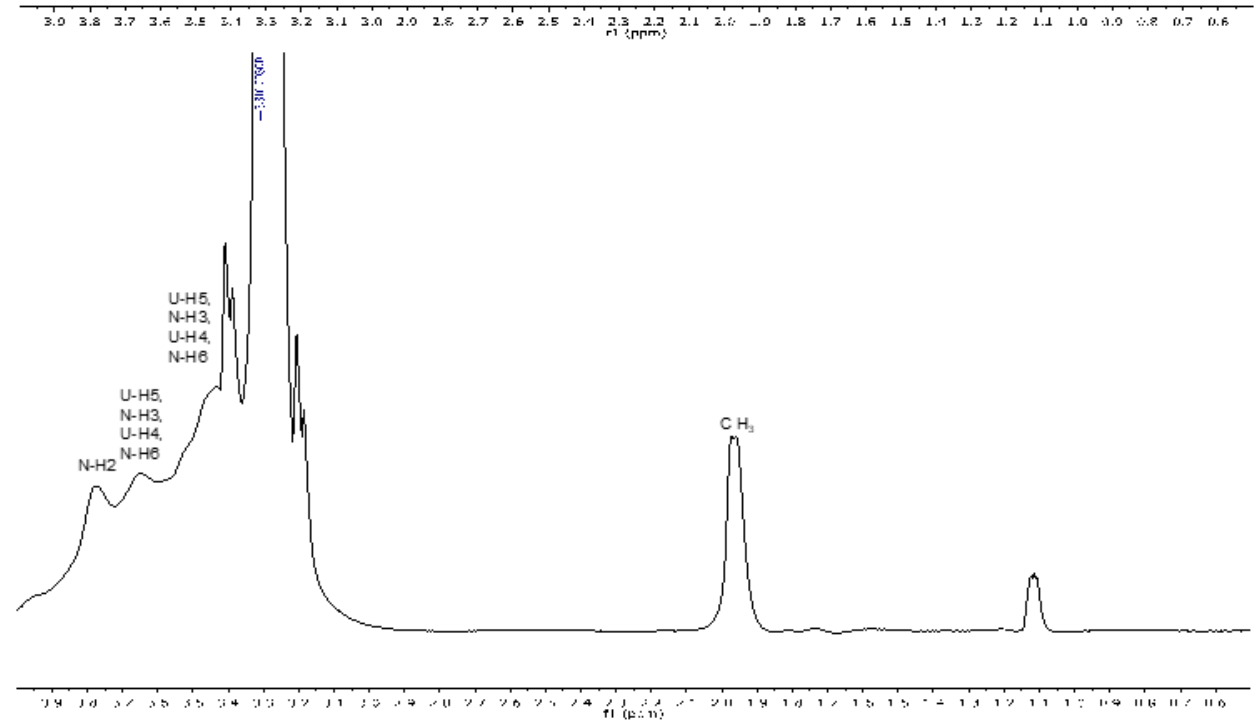

Figure 4-3. The NMR spectra of a) PC, b) HA, and c) PC mixed with HA. 
changes when PC was mixed with ultrasonicated HA showed trends similar to those seen when non-sonicated HA was added. However, the resonances for the PC acyl chain protons were detectable. The resonances corresponding to protons in the choline headgroup of PC overlapped with HA-related resonances and could not be resolved.

\section{Changes in RIE for glucose in the presence of HA}

Aqueous solutions containing glucose alone as well as glucose and HA were analyzed using MALDI-TOF/MS by premixing the solutions with DHB (see Fig. 4-4). When glucose was analyzed with the matrix only, the RIE was low $(7.5 \pm 0.9) \times 10^{-3}$ and the reproducibility was poor. These factors were also reflected by the S/N of $45 \pm 30$. Low sensitivity and precision were also observed for glucose in the presence of PLs as seen in Figure 4-5. Indeed, the RIE for Glu was only $(8.4 \pm 0.3) \times 10^{-3}$ when PC was present. However, when glucose was premixed with HA, the ionization efficiency increased significantly to $0.21 \pm 0.11\left(\rho=8.1 \times 10^{-5}\right.$, 1-way ANOVA). This led to an improvement in the $\mathrm{S} / \mathrm{N}$ for glucose, which increased significantly from $45 \pm 30$ (no HA) for glucose alone to $580 \pm 280$ when HA was present in the solution. A comparable improvement in the RIE of glucose $(0.15 \pm 0.09)$ was observed when the sample contained ultrasonicated HA. Further studies showed that the RIE of glucose was also enhanced when premixed with other negatively charged species such as phosphatidic acid (PA).

\section{Interactions in the porcine $\mathrm{VH}$}

A porcine $\mathrm{VH}$ was cut in half and each half was extracted using the Bligh and Dyer extraction method. One of the halves underwent ultrasonication after each addition of solvent whereas the second half was only lightly mixed after each addition of solvent. For the detection of PLs, PNA was used as the matrix. As shown in Figure 4-7, the mass 


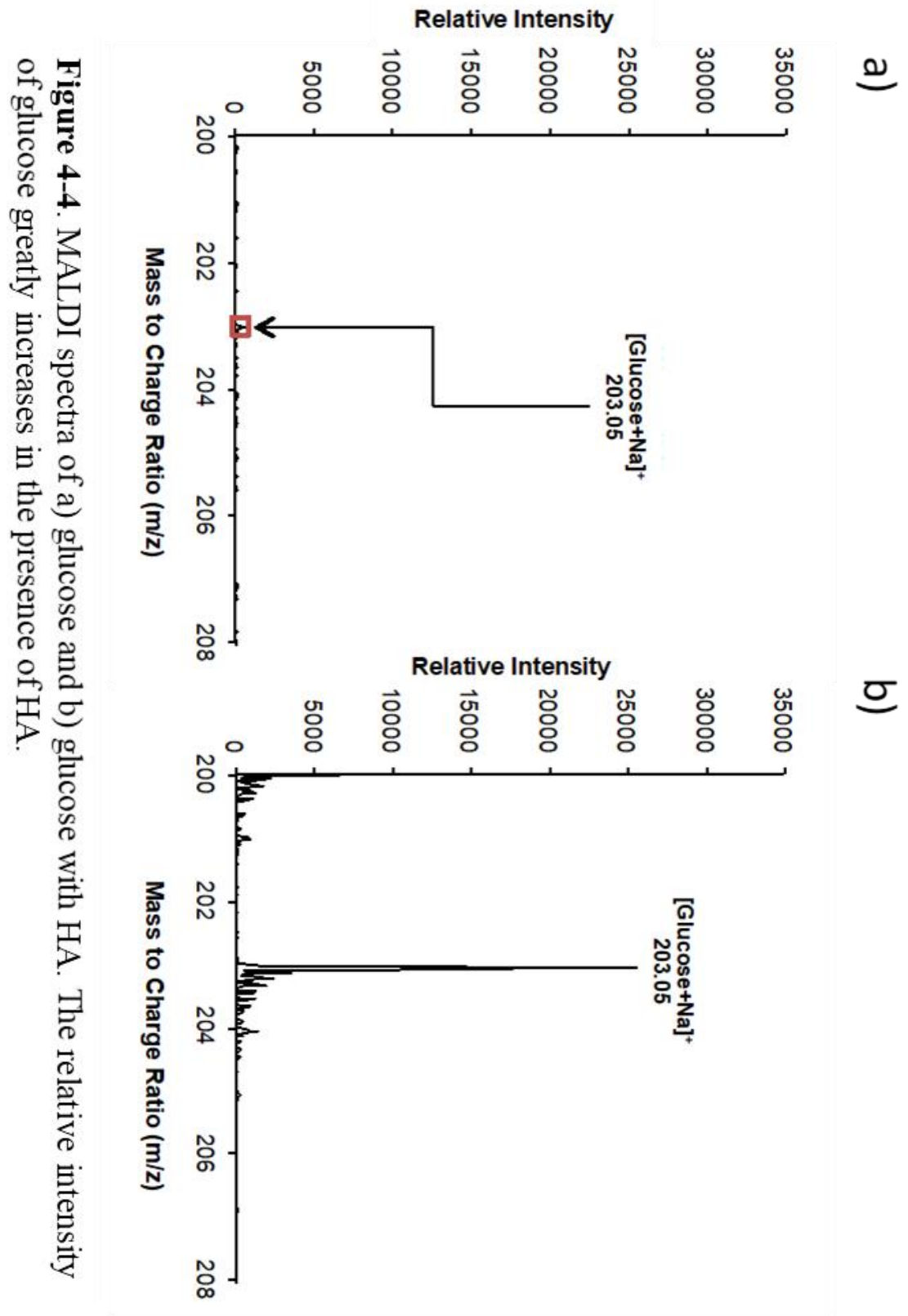




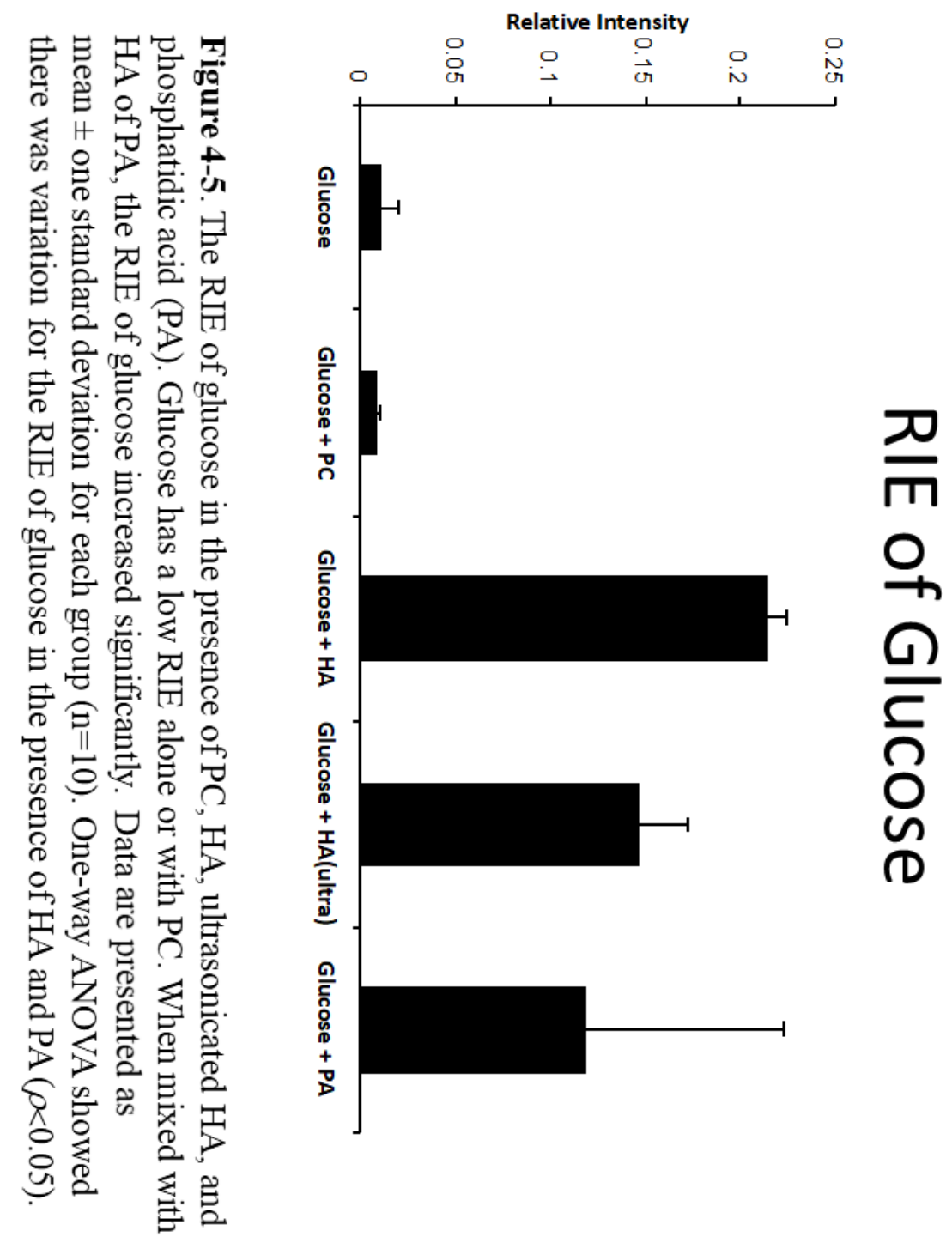


a)

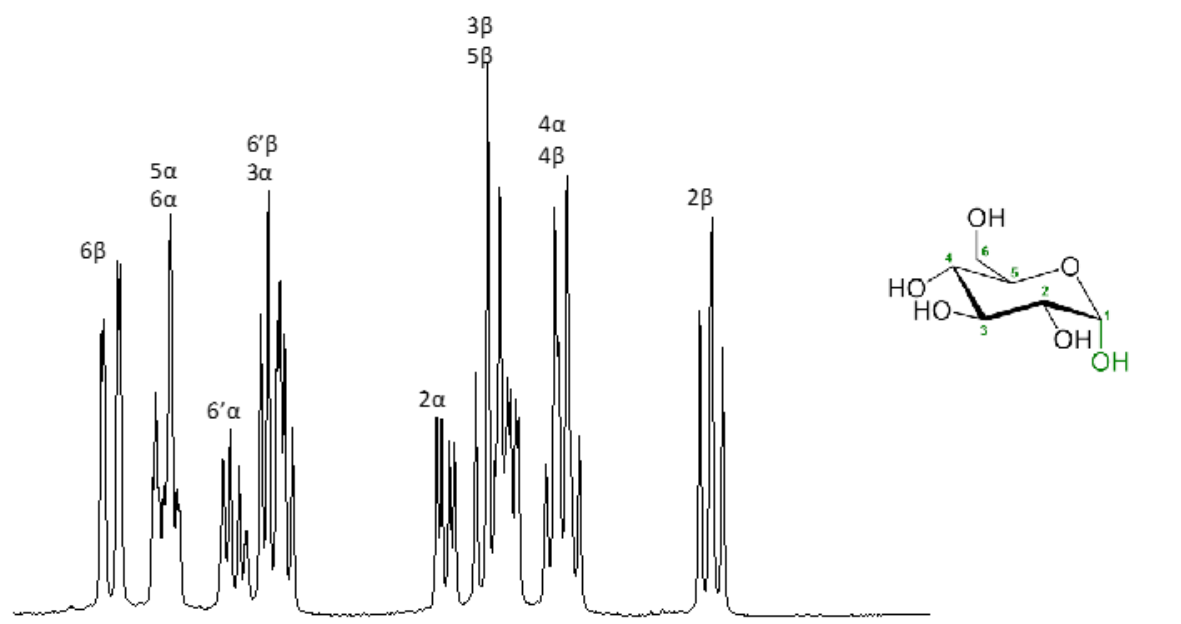

b)
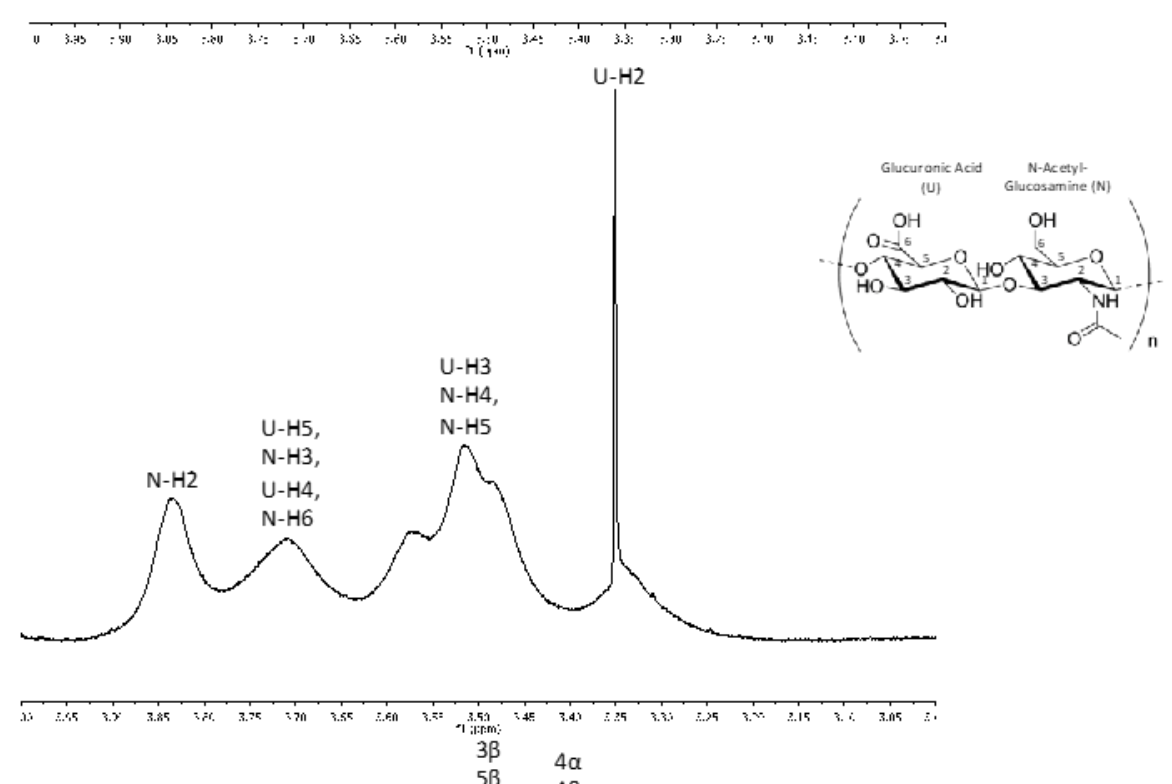

c)

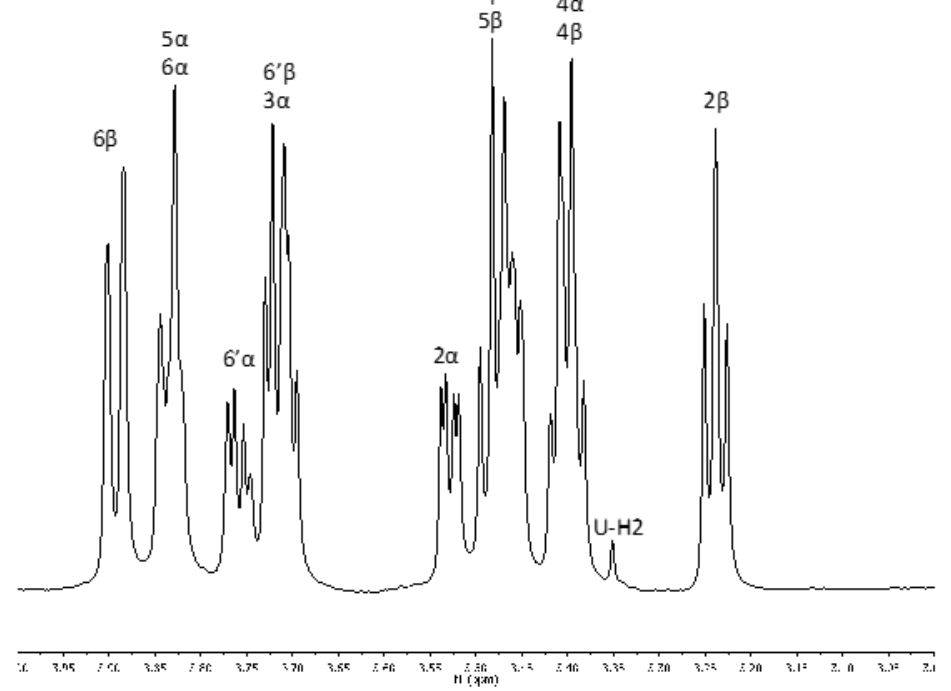

Figure 4-6. NMR spectra of a) glucose, b) HA, and c) mixture of glucose and HA. 
highest relative abundance followed by $\mathrm{PC}(32: 0)$ and $\mathrm{PC}(38: 4)$. PC(40:6) was only spectral data showed greater reduction in RIEs for PC-related peaks when the extract was ultrasonicated, but less with the non-sonicated VH. sonication. A similar trend was observed for all PLs; indeed lower RIEs and $\mathrm{S} / \mathrm{N}$ were seen in the presence of ultrasonicated VH rather than non-sonicated VH. The extracts showed PC(34:1) had the detectable in the presence of detected ultrasonicated VH. DMPC in known amounts was also added to the matrix-extract premix as a standard for quantitative comparisons, as seen in Figure 4-8. The normalized intensity of $\mathrm{PC}(32: 0)$ with no sonication $(0.46 \pm 0.06)$ was higher than that corresponding to ultrasonicated solutions $(0.094 \pm 0.022)$. The same trend was seen for PC(34:1) whose RIE decreased from $0.97 \pm 0.18$ without sonication to $0.25 \pm 0.06$ for the ultrasonicated ones.

A fresh porcine $\mathrm{VH}$ allowed for the detection of glucose using DHB as the matrix. Glucose was detected in the non-sonicated and the ultrasonicated samples. The S/N of glucose was $23 \pm 5.6$ with no sonication and decreased to $9.6 \pm 1.5$ with ultrasonication. This trend is similar to that observed in the model studies of glucose with HA and ultrasonicated HA.

The addition of the relative intensities of all PL signals gave higher values in the non-ultrasonicated samples than in those that were ultrasonicated. This indicates that the ultrasonication is breaking down the collagen-HA biomatrix and the PLs can interact more effectively with the shorter, more exposed HA polymer chains resulting in greater blocking of the PL ionization sites.

\section{DISCUSSION}


a)

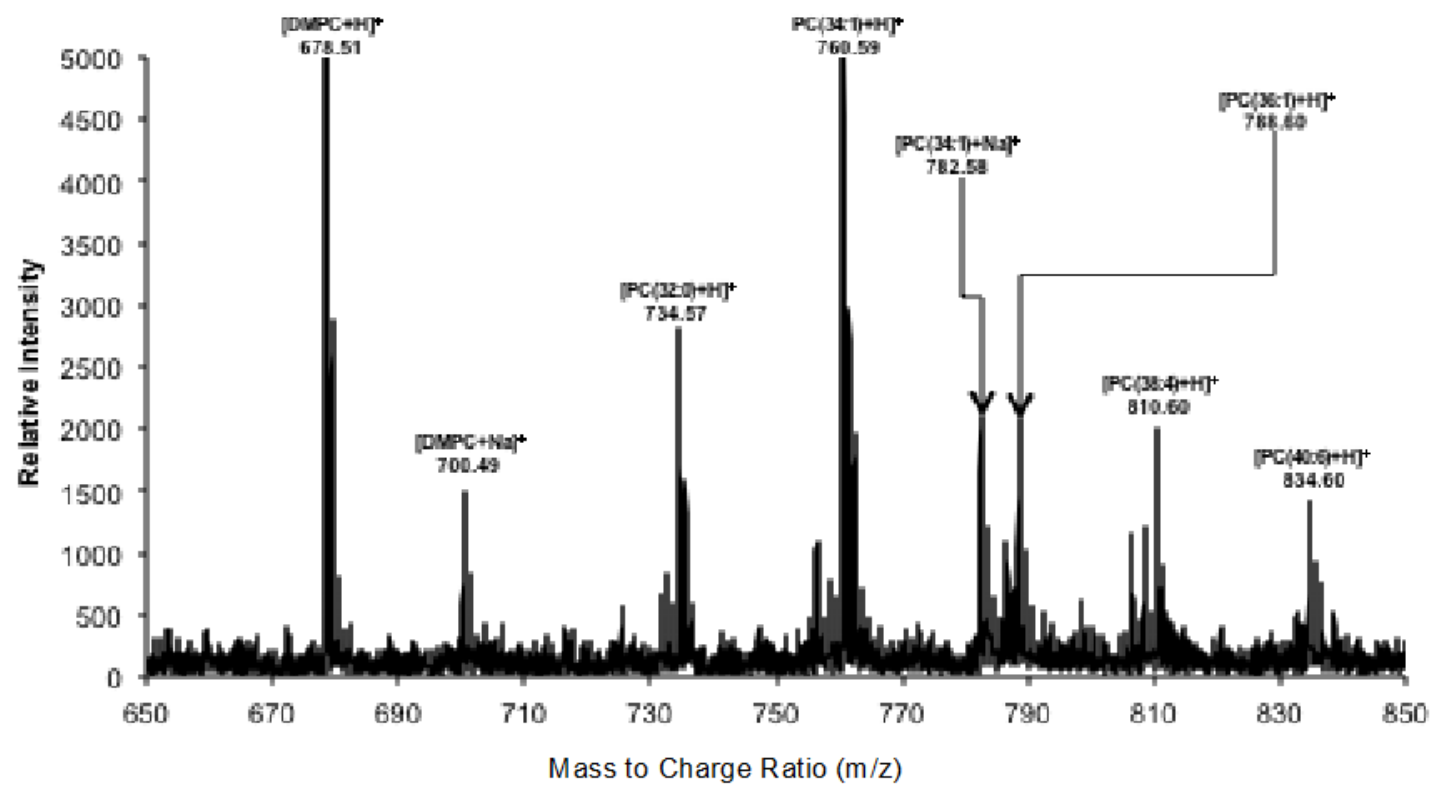

b)

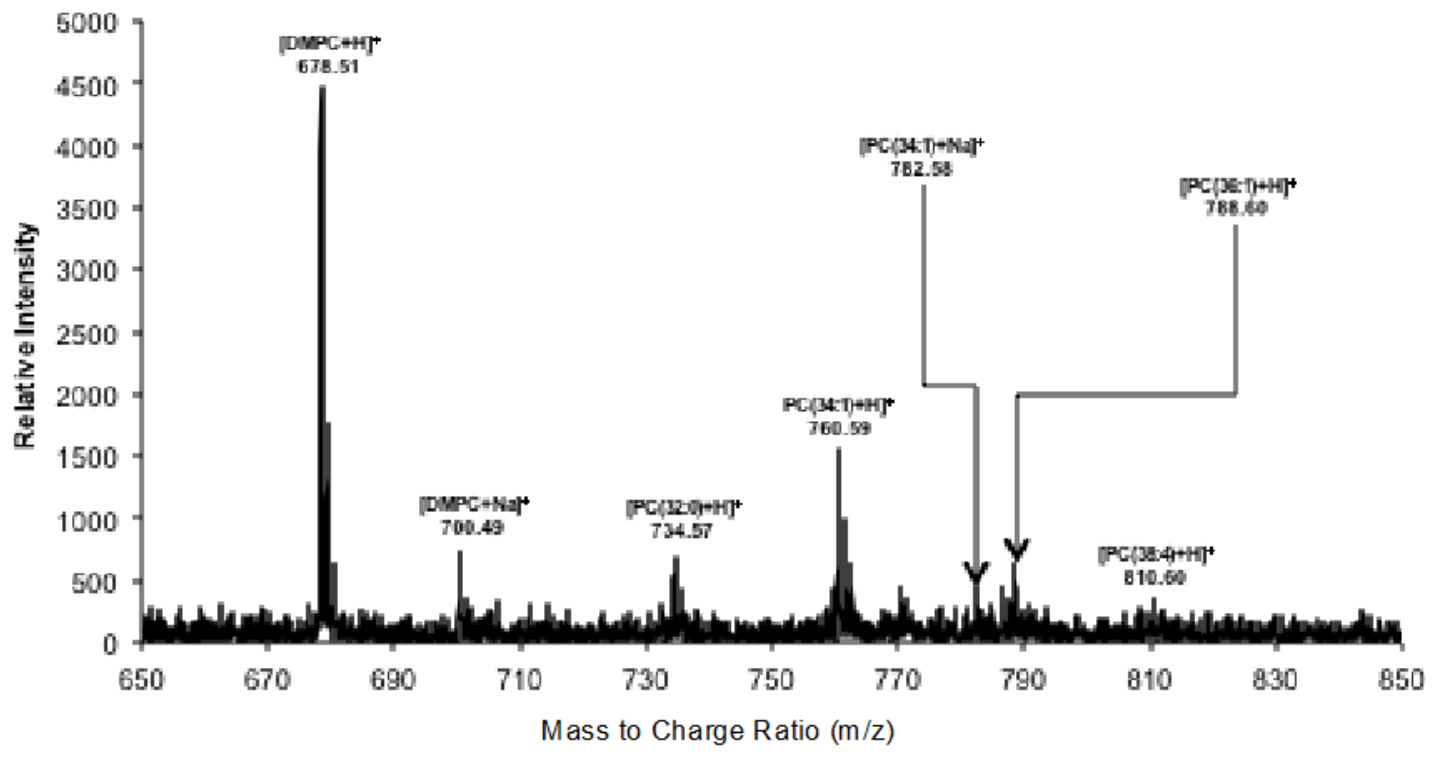

Figure 4-7. The MALDI spectra of an extracted porcine VH using the Bligh and Dyer method a) without sonication and b) with ultrasonication. DMPC was added as an internal calibrant to compare the relative abundance of PLs. The extraction with no sonication shows the highest relative abundance of PLs. 


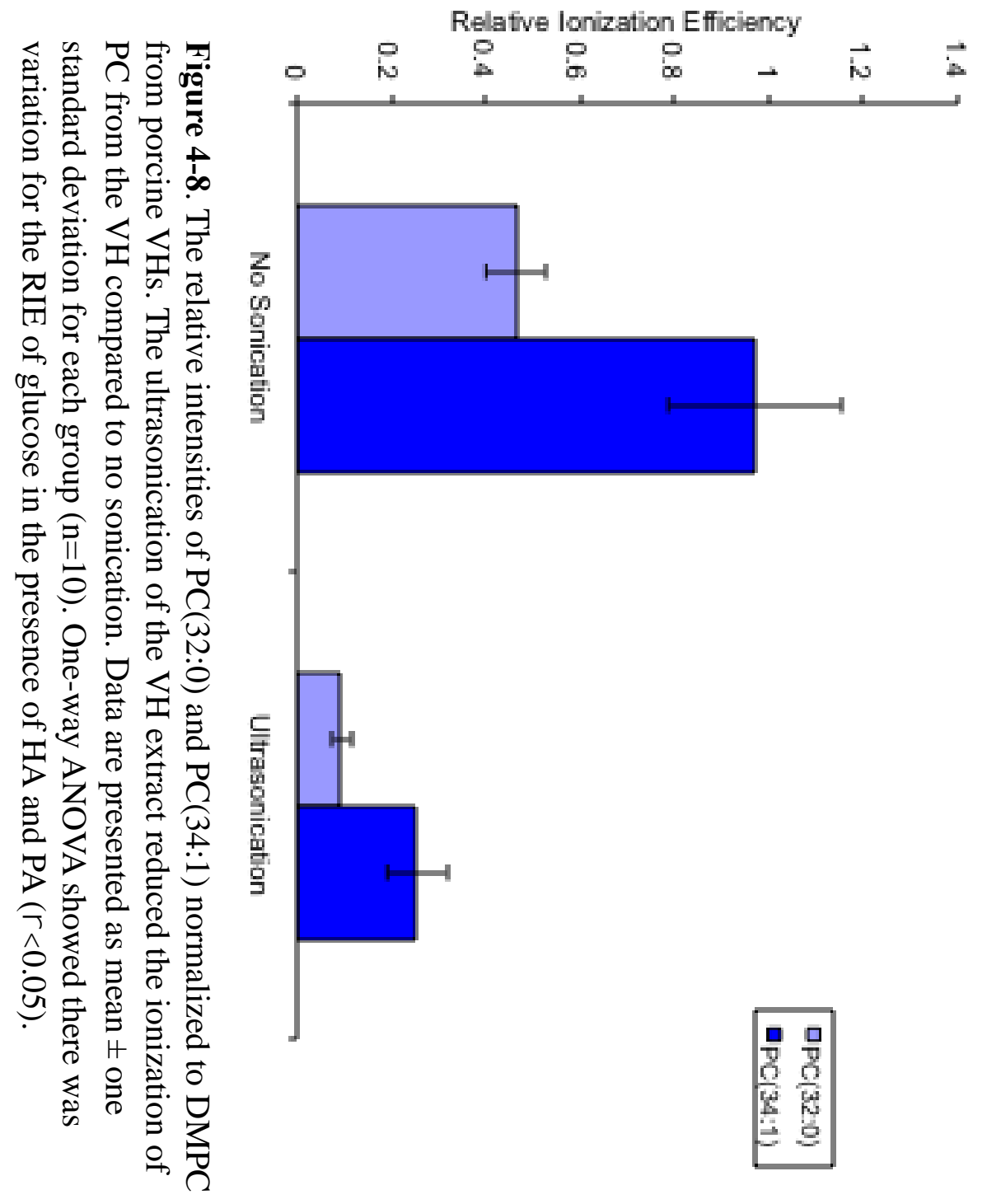




\section{Model Studies of PC and HA and/or Glucose}

The interaction of HA and PC has been previously explored and HA has been shown to interact with hydrophobic surfaces. ${ }^{170}$ Model studies have shown the RIE of PC is reduced in half when premixed with monomeric HA. ${ }^{166}$ It has been proposed that the acyl chains of the PC interact with the hydrophobic patches on the HA. ${ }^{14}$ However, HA has also been reported to interact with partially positive head group of PC and not with the negatively charged phosphoglycerol. ${ }^{171}$ Our model studies show that when HA and PC were mixed, the RIE of PC decreased. Adding ultrasonicated HA to PC decreased the RIE of PC even further which suggests when the HA is fragmented, it is more available for interactions with PC headgroups where the cationization occurs. The positively charged choline in the headgroup of PC is proposed to interact with the negatively charged carboxylate group of HA, which is more exposed after ultrasonication due to fragmentation of the polymeric HA. This results in the 'blocking' of the ionization site of PC. The acyl chains of PC also interact with the HA by twisting around the hydrophobic patches of the HA strands. This is supported by the NMR data showing the absence of resonances for protons in the PC acyl tails when PC is mixed with polymeric HA. This is the result of extreme broadening of these resonances due to a much faster transversal relaxation and suggests that he PC acyl tails can be intertwined with HA strands so that the hydrophobic patches of HA are in close proximity to the hydrophobic tails of PC. When the HA is fragmented the HA strands are not able for the PC acyl tails to twist around causing the acyl protons to be detected through NMR. Therefore, interactions between HA and PC do take place in liquid phase, as seen in the NMR results.

\section{Model Studies Glucose}


Model studies of glucose with HA show that in the presence of HA, the RIE of glucose increases. This significant increase suggests that glucose and HA interact allowing glucose to ionize more readily. NMR data also supports an interaction between the two. The data show a decrease in the $\mathrm{T}_{2}$ values of the protons on the 6' and 4 carbons as well as broadening of glucose resonances when HA is added. This suggests the interaction between glucose and HA involves the protons on the 6' and 4 carbons of the glucose molecule. It is mostly likely that the protons are interacting with the carboxylate group on the HA. The negative charge from the HA could be delocalized onto the glucose making it more electronegative and thus of greater attraction to positive ions. Similarly, when negatively-charged PA was present, the RIE for glucose increased. The data indicate that the negative charge of the PA headgroup also enhances the electonegativity of glucose thus making it easier to ionize by addition of a cation.

\section{VH tissue results}

Due to the VH containing $98 \%$ water, other vitreal components are present in very small concentrations that cannot be detected by NMR spectroscopy. However, MALDITOF/MS results showed that there are changes in the interactions of molecules with polymeric and fragmented HA. The ultrasonication of the extracts simulates liquefaction in the $\mathrm{VH}$. The porcine $\mathrm{VH}$ extracts that did not undergo ultrasonication showed that glucose and PC had a higher RIE than in the VH extraction that used ultrasonication. This suggests that the ultrasonication breaks down the HA and the rest of the biomatrix and allows the PLs to interact with the shortened HA strands, which reduce the ionization of PC. Further studies were conducted to confirm that the ultrasonication process alone did not cause the fragmentation of PLs. PC was ultrasonicated and was compared to PC not 
sonicated and showed similar RIEs and relative intensities. Since the ultrasonication has no effect on the ionization of PLs, the changes are due to a break down of the biomatrix causing an increase in the interactions between the biomatrix, specifically the fragmented HA, and the PLs and inhibiting ionization of them.

\section{Model and Tissue comparisons}

The results from the model and tissue studies are in agreement. They both showed that there are interactions between PC and HA as well as glucose and HA supported by changes in RIE. The model studies showed that there was a reduction in RIE of PC and glucose when mixed with polymeric HA compared to fragmented HA. The reduced RIE is repeated in the tissue study of ultrasonicated VH extracts when the RIE of PC is reduced compared to extracts that were not sonicated. This trend is also seen with glucose comparing the $\mathrm{VH}$ stamp versus the ultrasonicated stamp and observing a decrease in the RIE.

\section{CONCLUSIONS}

The interactions of vitreal components are of high interest when exploring possible causes of liquefaction. Interactions of HA-collagen-water give the VH a gel-like consistency and when this network is disrupted, liquefaction occurs. Interactions with hyaluronan and other vitreal components were explored to determine what effect they could have on disrupting this gelatinous network.

We determined that PC and HA interact based on reduced RIE using MALDI/MS and changes in chemical shift using NMR. Hydrophobic interactions between the hydrophobic patches of HA helices and the acyl chains of PC as well as between the 
positively-charged choline moiety of PC with the negatively charged carboxylate group of the deprotonated glucoronic acid of HA.

Glucose and HA also indicate an interaction based on the enhancement of RIE from MALDI/MS and peak broadening of glucose in the presence of HA using NMR. The data suggests an interaction occurs between the 6' and 4 hydroxyl groups on glucose and the carboxylate group on GlcA of HA.

Tissue studies indicate the ultrasonication of the VH biomatrix caused a reduction in the RIE of PC and glucose. This data agrees with model studies that RIEs of vitreal components are reduced when mixed with fragmented HA when compared to polymeric HA.

This work is pertinent to future studies of the VH. The biomatrix of the VH plays a role in the interactions of vitreal components and can be observed with changes in RIE using MALDI when NMR is not a viable option. With the information on the changes in RIE, the quantification of vitreal components can be more accurate for future studies. 


\title{
CHAPTER 5
}

\section{CONCLUSIONS AND FUTURE DIRECTIONS}

\begin{abstract}
Although the vitreous humor occupies $\sim 80 \%$ of the ocular volume, it is the least investigated component of the eye. Yet, its biological functions are very important: regulation of ocular growth and shape during development, prevention of migration of large macromolecules that could affect transparency and barrier for the diffusion of $\mathrm{O}_{2}$ that could cause oxidation of lenticular components. ${ }^{59}$ Despite its very high water content $(98 \%-99 \%)$, the $\mathrm{VH}$ is gelatinous but its consistency changes with age as it liquefies.
\end{abstract}

The projects described in this dissertation were designed to investigate the changes in molecular composition/structure that could contribute to liquefaction. MALDI/MS enabled the qualitative and quantitative analysis of regional changes in the levels of PLs and glucose in VHs from porcine and human eyes. In addition, both MALDI/MS and NMR spectroscopy have provided important and complementary information on the interactions between HA and PLs on glucose. This new knowledge is relevant to our understanding of vitreal liquefaction. The following paragraphs reiterate the main conclusions reached in the analytical and physiological aspects of this research.

\section{ANALYTICAL CONCLUSIONS}

Tissue Sectioning 
The 'stamp' method used for ex-situ studies gave fairly reproducible results and required no sample preparation thus preventing possible chemical alterations in the tissue. However, the sensitivity achieved with this approach was fairly low. For this reason, invitro studies were carried out for the regional analysis of PLs by dividing the $\mathrm{VH}$ in anterior, central and posterior regions. PLs were extracted using the Bligh and Dyer method. ${ }^{141}$ Higher sensitivity and reproducibility were achieved and more species were detected.

\section{Matrix Choices}

PNA was the best matrix to study PLs both ex-situ and in-vitro. However, for the detection of glucose, a more acidic matrix, DHB, was needed. Although DHB gave better results than PNA, the low ionization efficiency of glucose resulted in poor sensitivity and precision. Interestingly and surprisingly, larger glucose signals were detected when either HA or porcine VH was present. No eternal matrix was needed. Our hypothesis is that HA in the VH biomatrix acts as a 'biomatrix' that absorbs the laser radiation and transfers it to glucose allowing it to be ionized. Furthermore, and as presented in Chapter 4, the interaction(s) between HA and glucose appears to increase the electronegativity of glucose causing it to attract cations more effectively. This approach was proven unsuccessful for the detection of PLs.

\section{Impact of Molecular Interactions on Relative Ionization Efficiencies}

MALDI/MS is a sensitive and powerful tool for in vitro and ex situ imaging studies. However, another application of this method developed in this project is the characterization of molecular interactions inferred from changes in relative ionization 
efficiencies. The change in RIE values of the molecules showed that the ability to ionize was either enhanced or reduced depending on molecular associations. For example, the interaction of HA and PLs reduced the RIE of PC and SM. The positively charged choline headgroup in both PLs is proposed to interact with the negatively charged carboxylate of the HA molecule thus reducing the access of cations to the phosphate moiety of the headgroup. On the other hand, the interaction between HA and glucose was shown to enhance the RIE of glucose. The negative charge of the carboxylate group of HA could be delocalized onto the glucose by formation of H-bonds. As a result, glucose can be ionized more readily. This possibility was supported by the enhancement of the RIE of glucose when PA (in its anionic form) was added. It is proposed that the delocalization of the negative charge of the phosphate group of PA onto glucose by Hbond formation promotes the more effective ionization of glucose.

Another important trend observed in the model studies was the greater decrease in RIEs of PLs when fragment (rather than intact polymeric HA) was added. Similar, while the RIE was still higher in the presence of fragmented HA, the increase was not as significant as when intact HA was added. These changes in RIEs should be taken into account when quantitative studies are pursued in biological samples where the biomatrix can affect the degree of ionization of the analytes.

\section{PHYSIOLOGICAL CONCLUSIONS}

\section{Regional Distribution of Phospholipids and Glucose in Mammalian VHs}

Porcine and human VHs showed similar regional trends for the relative abundances of PLs. It was found that the posterior region of the $\mathrm{VH}$ has a significantly 
higher relative abundance of PLs compared to the anterior and central regions. Furthermore, the PL composition in the posterior region was found to be very similar to that of the retina. Higher levels of glucose were also detected in the posterior vitreal regions. Since the $\mathrm{VH}$ acts as a repository site after development, it is concluded that these compounds migrate from the retina into the $\mathrm{VH}$. Their diffusion within the $\mathrm{VH}$ is slow as shown by the low PL levels in the central region.

\section{Impact of Diabetes in the Levels of Phospholipids and Glucose in Human VHs}

In patients with diabetes, the BRB weakens allowing the migration of more molecules from the blood vessels into the retina and eventually into the $\mathrm{VH} .{ }^{172}$ The contents of both PLs and glucose were found to be significantly higher (around three times) in the $\mathrm{VH}$ of diabetics relative to the $\mathrm{VH}$ of non-diabetics of comparable age. The higher levels of PLs and glucose seen in the posterior regions of the VHs of diabetics are proposed to contribute to the accelerated liquefaction of the $\mathrm{VH}$. Indeed, liquefaction begins in the posterior region of the $\mathrm{VH}$, where the levels of PLs and glucose are the highest. It is proposed that the intercalation of either or both PLs and glucose within the HA/collagen/water network disrupt the tight mesh of cooperative H-bonds and cause the liquefaction of the gel.

\section{INTERACTIONS OF HA WITH PLs AND GLUCOSE}

Model studies showed that interactions take place between HA and PLs as well as between HA and glucose. These interactions could cause the collapse of the gelatinous network. The inclusion of PLs and glucose within the HA/collagen network could initiate 
the disruption of the extended hydrogen-bonding network and lead to liquefaction. A novel use of MALDI/MS was applied and showed that changes in RIE can be interpreted in terms of molecular associations that take place in the solvated sample but remain even after solvent evaporation.

\section{FUTURE STUDIES}

Future studies will focus on the inclusion of collagen to the biomatrix, age-related changes in $\mathrm{VH}$ from non-diabetics, and differences in the $\mathrm{VH}$ composition of diabetics affected by type I and II diabetes. The interactions among collagen and PLs, glucose, and HA will be explored to understand the potential role(s) played by collagen in the liquefaction process. It has been proposed that breakdown of collagen fibrils contributes to disruption of the gel-like network ${ }^{48,173}$.

The knowledge gained in these studies could lead to approaches that retard or eliminate liquefaction of the VH. This is the most challenging area of future research and it will require collaborative projects with bio-engineering as well as continued interaction with the Department of Ophthalmology. This is a worthwhile goal since by inhibiting the migration of PLs and glucose into the $\mathrm{VH}$ could prevent liquefaction and thus prevention of serious ocular diseases.

\section{CONCLUDING REMARKS}

Protocols were developed to overcome the challenges of working with VHs. Exsitu and in-vitro techniques were optimized in order to work with analytes in minute quantities within a highly hydrated ( $99 \%)$ medium. Under optimal conditions, porcine 
and human VHs were analyzed to determine regional composition and determine causes of liquefaction. The results showed high abundances of PLs and glucose in the posterior region, where vitreal liquefaction begins. The model studies confirm the interactions of PLs and glucose within the gel-like network of $\mathrm{HA} /$ collagen $/ \mathrm{H}_{2} \mathrm{O}$. Future studies need to be conducted to find ways to prevent the migration of compounds from the retina into the $\mathrm{VH}$ or even selectively remove these species from the $\mathrm{VH}$ to reverse the disruption of the vitreal network. 


\section{REFERENCES}

(1) Bishop, P. The Biochemical Structure of Mammalian Vitreous. Eye 1996, 10 (6), 664-670.

(2) Krause Ac. Chemistry of the Vitreous Humor: I. Chemical Composition of the Proteins. Arch. Ophthalmol. 1934, 11 (6), 960-963.

(3) Meyer, K.; Palmer, J. W. The Polysaccharide of the Vitreous Humor. J. Biol. Chem. 1934, 107 (3), 629-634.

(4) Simoni, R. D.; Hill, R. L.; Vaughan, M.; Hascall, V. The Discovery of Hyaluronan by Karl Meyer. J. Biol. Chem. 2002, 277 (39), e27-e27.

(5) Fakhari, A.; Berkland, C. Applications and Emerging Trends of Hyaluronic Acid in Tissue Engineering, as a Dermal Filler, and in Osteoarthritis Treatment. Acta Biomater. 2013, 9 (7), 7081-7092.

(6) Necas, J.; Bartosikova, L.; Brauner, P.; Kolar, J. Hyaluronic Acid (Hyaluronan): A Review. Vet. Med. (Praha) 2008.

(7) Theocharis, D. A.; Skandalis, S. S.; Noulas, A. V.; Papageorgakopoulou, N.; Theocharis, A. D.; Karamanos, N. K. Hyaluronan and Chondroitin Sulfate Proteoglycans in the Supramolecular Organization of the Mammalian Vitreous Body. Connect. Tissue Res. 2008, 49 (3), 124-128.

(8) Cowman, M. K.; Lee, H.-G.; Schwertfeger, K. L.; McCarthy, J. B.; Turley, E. A. The Content and Size of Hyaluronan in Biological Fluids and Tissues. Front. Immunol. 2015, 6 .

(9) Day, A.; Prestwich, G. Hyaluronan-Binding Proteins: Tying Up the Giant. J. Biol. Chem. 2001, No. 277, 4585-4588.

(10) Fraser, J. R. E.; Laurent, T. C.; Laurent, U. B. G. Hyaluronan: Its Nature, Distribution, Functions and Turnover. J. Intern. Med. 1997, 242 (1), 27-33.

(11) Hunger, J.; Bernecker, A.; Bakker, H. J.; Bonn, M.; Richter, R. P. Hydration Dynamics of Hyaluronan and Dextran. Biophys. J. 2012, 103 (1), L10-L12.

(12) Ivanov, D.; Neamtu, A. Molecular Dynamics Evaluation of Hyaluronan Interactions with Dimethylsilanediol in Aqueous Solution. Rev. Roum. Chim. 2013, 58, 229-238.

(13) Almond, A.; Sheehan, J. K.; Brass, A. Molecular Dynamics Simulations of the Two Disaccharides of Hyaluronan in Aqueous Solution. Glycobiology 1997, 7 (5), 597-604.

(14) Ghosh, P.; Hutadilok, N.; Adam, N.; Lentini, A. Interactions of Hyaluronan (Hyaluronic Acid) with Phospholipids as Determined by Gel Permeation Chromatography, Multi-Angle Laser-Light-Scattering Photometry and 1H-NMR Spectroscopy. Int. J. Biol. Macromol. 1994, 16 (5), 237-244.

(15) Pasquali-Ronchetti, I.; Quaglino, D.; Mori, G.; Bacchelli, B.; Ghosh, P. Hyaluronan-Phospholipid Interactions. J. Struct. Biol. 1997, 120 (1), 1-10. 
(16) Laurent, T. C.; Laurent, U. B.; Fraser, J. R. E. The Structure and Function of Hyaluronan: An Overview. Immunol. Cell Biol. 1996, 74 (2), A1-A7.

(17) Scott, J. E. Proteoglycan-Fibrillar Collagen Interactions. Biochem. J. 1988, 252 (2), 313-323.

(18) McDevitt, C. A.; Marcelino, J.; Tucker, L. Interaction of Intact Type VI Collagen with Hyaluronan. FEBS Lett. 1991, 294 (3), 167-170.

(19) Kielty, C. M.; Whittaker, S. P.; Grant, M. E.; Shuttleworth, C. A. Type VI Collagen Microfibrils: Evidence for a Structural Association with Hyaluronan. $J$. Cell Biol. 1992, 118 (4), 979-990.

(20) Sebag, J. The Vitreous; Springer-Verlag, 1989.

(21) Scott, J. E.; Heatley, F. Hyaluronan Forms Specific Stable Tertiary Structures in Aqueous Solution: A 13C NMR Study. Proc. Natl. Acad. Sci. 1999, 96 (9), 48504855.

(22) David Nelson; Michael Cox. Principles of Biochemistry, 6th ed.

(23) Le Goff, M. M.; Bishop, P. N. Adult Vitreous Structure and Postnatal Changes. Eye 2008, 22 (10), 1214-1222.

(24) Gelse, K.; Pöschl, E.; Aigner, T. Collagens — structure, Function, and Biosynthesis. Adv. Drug Deliv. Rev. 2003, 55 (12), 1531-1546.

(25) Gregory, K. E.; Oxford, J. T.; Chen, Y.; Gambee, J. E.; Gygi, S. P.; Aebersold, R.; Neame, P. J.; Mechling, D. E.; Bächinger, H. P.; Morris, N. P. Structural Organization of Distinct Domains within the Non-Collagenous N-Terminal Region of Collagen Type XI. J. Biol. Chem. 2000, 275 (15), 11498-11506.

(26) Bos, K. J.; Holmes, D. F.; Kadler, K. E.; McLeod, D.; Morris, N. P.; Bishop, P. N. Axial Structure of the Heterotypic Collagen Fibrils of Vitreous Humour and cartilage1. J. Mol. Biol. 2001, 306 (5), 1011-1022.

(27) Bishop, P. N.; Holmes, D. F.; Kadler, K. E.; McLeod, D.; Bos, K. J. Age-Related Changes on the Surface of Vitreous Collagen Fibrils. Investig. Opthalmology Vis. Sci. 2004, 45 (4), 1041.

(28) Blaschke, U. K.; Eikenberry, E. F.; Hulmes, D. J. S.; Galla, H.-J.; Bruckner, P. Collagen XI Nucleates Self-Assembly and Limits Lateral Growth of Cartilage Fibrils. J. Biol. Chem. 2000, 275 (14), 10370-10378.

(29) Kokavec, S. H. M. J. Biochemical Analysis of the Living Human Vitreous. Clin. Amp Exp. Ophthalmol. 2016.

(30) D'Asaro, B. S. BIOCHEMISTRY OF THE EYE: III. Lipids of the Lens, Cornea, Iris, Ciliary Body, and Retina. AMA Arch. Ophthalmol. 1954, 51 (5), 596.

(31) Krause AC. Chemistry of the Vitreous Humor: Iii. Lipids. Arch. Ophthalmol. 1935, 13 (6), 1022-1025.

(32) Kim, J. O.; Cotlier, E. Phospholipid Distributions and Fatty Acid Composition of Lysophosphatidylcholine and Phosphatidylcholine in Rabbit Aqueous Humor, Lens and Vitreous. Exp. Eye Res. 1976, 22 (6), 569-576.

(33) Reddy, D. V. N.; Kinsey, V. E. Composition of the Vitreous Humor in Relation to That of Plasma and Aqueous Humors. Arch. Ophthalmol. 1960, 63 (4), 715-720.

(34) Reardon, A.; Heinegård, D.; McLeod, D.; Sheehan, J. K.; Bishop, P. N. The Large Chondroitin Sulphate Proteoglycan Versican in Mammalian Vitreous. Matrix Biol. J. Int. Soc. Matrix Biol. 1998, 17 (5), 325-333. 
(35) Wight, T. N. Versican: A Versatile Extracellular Matrix Proteoglycan in Cell Biology. Curr. Opin. Cell Biol. 2002, 14 (5), 617-623.

(36) Gao, B.; Chen, X.; Timothy, N.; Aiello, L. Characterization of the Vitreous Proteome in Diabetes without Diabetic Retinopathy and Diabetes with Proliferative Diabetic Retinopathy. J. Proteome Res. 2008, No. 7, 2516-2525.

(37) Takano, S.; Ishiwata, S.; Nakazawa, M.; Mizugaki, M.; Tamai, M. Determination of Ascorbic Acid in Human Vitreous Humor by High-Performance Liquid Chromatography with UV Detection. Curr. Eye Res. 1997, 16 (6), 589-594.

(38) Kokavec, J.; Min, S. H.; Tan, M. H.; Gilhotra, J. S.; Newland, H. S.; Durkin, S. R.; Grigg, J.; Casson, R. J. Biochemical Analysis of the Living Human Vitreous. Clin. Experiment. Ophthalmol. 2016, 44 (7), 597-609.

(39) Lundquist, O.; Österlin, S. Glucose Concentration in the Vitreous of Nondiabetic and Diabetic Human Eyes. Graefes Arch. Clin. Exp. Ophthalmol. 1994, 232 (2), 71-74.

(40) Freddo, T. F.; Chaum, E. Anatomy of the Eye and Orbit: The Clinical Essentials; Lippincott Williams \& Wilkins, 2017.

(41) Sebag, J. Ageing of the Vitreous. Eye 1987, 1 (2), 254-262.

(42) Forrester, J. V.; Dick, A. D.; McMenamin, P. G.; Roberts, F.; Pearlman, E. The Eye E-Book: Basic Sciences in Practice; Elsevier Health Sciences, 2015.

(43) Richer, S. P.; Rose, R. C. Water Soluble Antioxidants in Mammalian Aqueous Humor: Interaction with UV B and Hydrogen Peroxide. Vision Res. 1998, 38 (19), 2881-2888.

(44) Holekamp, N. Vitreous Research: The Oxygen Story.

(45) A. Pirie. The Effect of Hyaluronidase Injection on the Vitreous Humor of the Rabbit.

(46) Deguine, V.; Menasche, M.; Ferrari, P.; Fraisse, L.; Pouliquen, Y.; Robert, L. Free Radical Depolymerization of Hyaluronan by Maillard Reaction Products: Role in Liquefaction of Aging Vitreous. Int. J. Biol. Macromol. 1998, 22 (1), $17-$ 22.

(47) Los, L. I.; van der Worp, R. J.; van Luyn, M. J. A.; Hooymans, J. M. M. AgeRelated Liquefaction of the Human Vitreous Body: LM and TEM Evaluation of the Role of Proteoglycans and Collagen. Investig. Opthalmology Vis. Sci. 2003, 44 (7), 2828.

(48) Bishop, P. N. Structural Macromolecules and Supramolecular Organisation of the Vitreous Gel. Prog. Retin. Eye Res. 2000, 19 (3), 323-344.

(49) Tan, L. E.; Orilla, W.; Hughes, P. M.; Tsai, S.; Burke, J. A.; Wilson, C. G. Effects of Vitreous Liquefaction on the Intravitreal Distribution of Sodium Fluorescein, Fluorescein Dextran, and Fluorescent Microparticles. Invest. Ophthalmol. Vis. Sci. 2011, 52 (2), 1111-1118.

(50) Lumi, X.; Hawlina, M.; Glavač, D.; Facskó, A.; Moe, M. C.; Kaarniranta, K.; Petrovski, G. Ageing of the Vitreous: From Acute Onset Floaters and Flashes to Retinal Detachment. Ageing Res. Rev. 2015, 21, 71-77.

(51) Abdelkawi, S. A.; Abdel-Salam, A. M.; Ghoniem, D. F.; Ghaly, S. K. Vitreous Humor Rheology after Nd:YAG Laser Photo Disruption. Cell Biochem. Biophys. 2014, 68 (2), 267-274. 
(52) Balazs, E. A. The Role of Hyaluronan in the Structure and Function of the Biomatrix of Connective Tissues. Struct. Chem. 2009, 20 (2), 233-243.

(53) Huang, D.; Swanson, E. A.; Lin, C. P.; Schuman, J. S.; Stinson, W. G.; Chang, W.; Hee, M. R.; Flotte, T.; Gregory, K.; Puliafito, C. A.; et al. Optical Coherence Tomography. Science 1991, 254 (5035), 1178-1181.

(54) Gentile, R. C.; Landa, G.; Pons, M. E.; Eliott, D.; Rosen, R. B. Macular Hole Formation, Progression, and Surgical Repair: Case Series of Serial Optical Coherence Tomography and Time Lapse Morphing Video Study. BMC Ophthalmol. 2010, 10, 24.

(55) M.D, D. A. B.; M.D, C. P. W. Retinal Detachment: Priniciples and Practice; Oxford University Press, 2009.

(56) Rodriguez-Peralta, L. A. Hematic and Fluid Barriers of the Retina and Vitreous Body. J. Comp. Neurol. 1968, 132 (1), 109-123.

(57) Chen, M.-S.; Hou, P.-K.; Tai, T.-Y.; Lin, B. J. Blood-Ocular Barriers. Tzu Chi Med. J. 2008, 20 (1), 25-34.

(58) Cunha-vaz, J. G. The Blood-Ocular Barriers: Past, Present, and Future. Doc. Ophthalmol. 93 (1-2), 149-157.

(59) Sebag, J. The Vitreous; Springer-Verlag, 1989.

(60) Sander, B.; Larsen, M.; Engler, C.; Moldow, B.; Larsen, N. Diabetic Macular Oedema: A Comparison of Vitreous Fluorometry, Angiography, and Retinopathy. Br. J. Ophthalmol. 86 (3), 316-320.

(61) Maritim, A. C.; Sanders, R. A.; Watkins, J. B. Diabetes, Oxidative Stress, and Antioxidants: A Review. J. Biochem. Mol. Toxicol. 2003, 17 (1), 24-38.

(62) Kowluru, R. A.; Mishra, M. Oxidative Stress, Mitochondrial Damage and Diabetic Retinopathy. Biochim. Biophys. Acta BBA - Mol. Basis Dis. 2015, 1852 (11), 2474-2483.

(63) Loukovaara, S.; Nurkkala, H.; Tamene, F.; Gucciardo, E.; Liu, X.; Repo, P.; Lehti, K.; Varjosalo, M. Quantitative Proteomics Analysis of Vitreous Humor from Diabetic Retinopathy Patients. J. Proteome Res. 2015, 14 (12), 5131-5143.

(64) Jedziniak, J. A.; Chylack, L. T.; Cheng, H. M.; Gillis, M. K.; Kalustian, A. A.; Tung, W. H. The Sorbitol Pathway in the Human Lens: Aldose Reductase and Polyol Dehydrogenase. Invest. Ophthalmol. Vis. Sci. 1981, 20 (3), 314-326.

(65) Barba, I.; Garcia-Ramírez, M.; Hernández, C.; Alonso, M. A.; Masmiquel, L.; García-Dorado, D.; Simó, R. Metabolic Fingerprints of Proliferative Diabetic Retinopathy: An 1H-NMR-Based Metabonomic Approach Using Vitreous Humor. Invest. Ophthalmol. Vis. Sci. 2010, 51 (9), 4416-4421.

(66) Blair, N. P.; Zeimer, R. C.; Rusin, M. M.; Cunha-Vaz, J. G. Outward Transport of Fluorescein from the Vitreous in Normal Human Subjects. Arch. Ophthalmol. Chic. Ill 1960 1983, 101 (7), 1117-1121.

(67) DiMattio, J. In Vivo Use of Neutral Radiolabelled Molecular Probes to Evaluate Blood-Ocular Barrier Integrity in Normal and Streptozotocin-Diabetic Rats | SpringerLink.

(68) Ferrante do Amaral, C. E.; Wolf, B. Current Development in Non-Invasive Glucose Monitoring. Med. Eng. Phys. 2008, 30 (5), 541-549. 
(69) Géhl, Z.; Bakondi, E.; Resch, M. D.; Hegedüs, C.; Kovács, K.; Lakatos, P.; Szabó, A.; Nagy, Z.; Virág, L. Diabetes-Induced Oxidative Stress in the Vitreous Humor. Redox Biol. 2016, 9, 100-103.

(70) Sparvero, L.; Amoscato, A.; Kochanek, P.; Pitt, B.; Kagan, V.; Bayır, H. MassSpectrometry Based Oxidative Lipidomics and Lipid Imaging: Applications in Traumatic Brain Injury. J. Neurochem. 2010, 115 (6), 1322-1336.

(71) Spickett, C. M.; Pitt, A. R. Oxidative Lipidomics Coming of Age: Advances in Analysis of Oxidized Phospholipids in Physiology and Pathology. Antioxid. Redox Signal. 2015, 22 (18), 1646-1666.

(72) Reis, A.; Spickett, C. M. Chemistry of Phospholipid Oxidation. Biochim. Biophys. Acta BBA - Biomembr. 2012, 1818 (10), 2374-2387.

(73) Zhang, Y.; Cocklin, R. R.; Bidasee, K. R.; Wang, M. Rapid Determination of Advanced Glycation End Products of Proteins Using MALDI-TOF-MS and PERL Script Peptide Searching Algorithm. J. Biomol. Tech. JBT 2003, 14 (3), 224-230.

(74) Rabbani, N.; Ashour, A.; Thornalley, P. J. Mass Spectrometric Determination of Early and Advanced Glycation in Biology. Glycoconj. J. 2016, 33 (4), 553-568.

(75) Nagaraj, R. H.; Linetsky, M.; Stitt, A. W. The Pathogenic Role of Maillard Reaction in the Aging Eye. Amino Acids 2012, 42 (4), 1205-1220.

(76) Nowotny, K.; Jung, T.; Höhn, A.; Weber, D.; Grune, T. Advanced Glycation End Products and Oxidative Stress in Type 2 Diabetes Mellitus. Biomolecules 2015, 5 (1), 194-222.

(77) Sebag, J. Imaging Vitreous. Eye 2002, 16 (4), 429-439.

(78) Aguayo, J.; Glaser, B.; Mildvan, A.; Cheng, H. M.; Gonzalez, R. G.; Brady, T. Study of Vitreous Liquifaction by NMR Spectroscopy and Imaging. Invest. Ophthalmol. Vis. Sci. 1985, 26 (5), 692-697.

(79) Sebag, J. Seeing the Invisible: The Challenge of Imaging Vitreous. J. Biomed. Opt. 2004, 9 (1), 38-46.

(80) Estrada, R.; Stolowich, N.; Yappert, M. C. Influence of Temperature on 31P NMR Chemical Shifts of Phospholipids and Their Metabolites I. In Chloroformmethanol-water. Anal. Biochem. 2008, 380 (1), 41-50.

(81) Borchman, D.; Byrdwell, W. C.; Yappert, M. C. Regional and Age-Dependent Differences in the Phospholipid Composition of Human Lens Membranes. Invest. Ophthalmol. Vis. Sci. 1994, 35 (11), 3938-3942.

(82) Borchman, D.; Ozaki, Y.; Lamba, O. P.; Byrdwell, W. C.; Yappert, M. C. Age and Regional Structural Characterization of Lipid Hydrocarbon Chains from Human Lenses by Infrared, and near-Infrared Raman, Spectroscopies. Biospectroscopy 1996, 2 (2), 113-123.

(83) Greiner, J. V.; Auerbach, D. B.; Leahy, C. D.; Glonek, T. Distribution of Membrane Phospholipids in the Crystalline Lens. Invest. Ophthalmol. Vis. Sci. 1994, 35 (10), 3739-3746.

(84) Berry, K. A. Z.; Gordon, W. C.; Murphy, R. C.; Bazan, N. G. Spatial Organization of Lipids in the Human Retina and Optic Nerve by MALDI Imaging Mass Spectrometry. J. Lipid Res. 2014, 55 (3), 504-515. 
(85) Estrada, R.; Yappert, M. C. Regional Phospholipid Analysis of Porcine Lens Membranes by Matrix-Assisted Laser Desorption/Ionization Time-of-Flight Mass Spectrometry. J. Mass Spectrom. JMS 2004, 39 (12), 1531-1540.

(86) Grey, A. C.; Chaurand, P.; Caprioli, R. M.; Schey, K. L. MALDI Imaging Mass Spectrometry of Integral Membrane Proteins from Ocular Lens and Retinal Tissue. J. Proteome Res. 2009, 8 (7), 3278-3283.

(87) Grey, A. C. MALDI Imaging of the Eye: Mapping Lipid, Protein and Metabolite Distributions in Aging and Ocular Disease. Int. J. Mass Spectrom. 2016, 401, $31-$ 38.

(88) Ly, A.; Schöne, C.; Becker, M.; Rattke, J.; Meding, S.; Aichler, M.; Suckau, D.; Walch, A.; Hauck, S. M.; Ueffing, M. High-Resolution MALDI Mass Spectrometric Imaging of Lipids in the Mammalian Retina. Histochem. Cell Biol. 2014, 143 (5), 453-462.

(89) Palmer, A. D.; Griffiths, R.; Styles, I.; Claridge, E.; Calcagni, A.; Bunch, J. Sucrose Cryo-Protection Facilitates Imaging of Whole Eye Sections by MALDI Mass Spectrometry. J. Mass Spectrom. 2012, 47 (2), 237-241.

(90) Vidová, V.; Pól, J.; Volný, M.; Novák, P.; Havlíček, V.; Wiedmer, S. K.; Holopainen, J. M. Visualizing Spatial Lipid Distribution in Porcine Lens by MALDI Imaging High-Resolution Mass Spectrometry. J. Lipid Res. 2010, 51 (8), 2295-2302.

(91) Harris, D. Quantitative Chemical Analysis, 7th ed.; W. H. Freeman, 2006.

(92) Siuzdak, G. An Introduction to Mass Spectrometry Ionization: An Excerpt from The Expanding Role of Mass Spectrometry in Biotechnology, 2nd Ed.; MCC Press: San Diego, 2005. JALA J. Assoc. Lab. Autom. 2004, 9 (2), 50-63.

(93) Skoog, D. A.; Holler, F. J.; Crouch, S. R. Principles of Instrumental Analysis; Thomson Brooks/Cole, 2007.

(94) Becker, K. H.; Tarnovsky, V. Electron-Impact Ionization of Atoms, Molecules, Ions and Transient Species. Plasma Sources Sci. Technol. 1995, 4 (2), 307.

(95) Munson, M. S. B.; Field, F. H. Chemical Ionization Mass Spectrometry. I. General Introduction. J. Am. Chem. Soc. 1966, 88 (12), 2621-2630.

(96) Field, F. H. Chemical Ionization Mass Spectrometry. 1968, 1, 42-49.

(97) Ho, C.; Lam, C.; Chan, M.; Cheung, R.; Law, L.; Lit, L.; Ng, K.; Suen, M.; Tai, H. Electrospray Ionisation Mass Spectrometry: Principles and Clinical Applications. Clin. Biochem. Rev. 2003, 24 (1), 3-12.

(98) Fenn, J. B.; Mann, M.; Meng, C. K.; Wong, S. F.; Whitehouse, C. M. Electrospray Ionization-principles and Practice. Mass Spectrom. Rev. 9 (1), $37-$ 70.

(99) Ito, Y.; Takeuchi, T.; Ishii, D.; Goto, M. Direct Coupling of Micro HighPerformance Liquid Chromatography with Fast Atom Bombardment Mass Spectrometry. J. Chromatogr. A 1985, 346, 161-166.

(100) Barber, M.; Bordoli, R. S.; Sedgwick, R. D.; Tyler, A. N. Fast Atom Bombardment of Solids as an Ion Source in Mass Spectrometry. Nature 1981, 293 (5830), 270-275.

(101) Zalm, P. C. Secondary Ion Mass Spectrometry. 1994, 45, 753-772. 
(102) Brunelle, A.; Touboul, D.; Laprévote, O. Biological Tissue Imaging with Timeof-Flight Secondary Ion Mass Spectrometry and Cluster Ion Sources. J. Mass Spectrom. 40 (8), 985-999.

(103) Takáts, Z.; Wiseman, J. M.; Gologan, B.; Cooks, R. G. Mass Spectrometry Sampling Under Ambient Conditions with Desorption Electrospray Ionization. Science 2004, 306 (5695), 471-473.

(104) Hoffmann, E. de. Mass Spectrometry. In Kirk-Othmer Encyclopedia of Chemical Technology; American Cancer Society, 2005.

(105) Mellon, F. A. MASS SPECTROMETRY | Principles and Instrumentation. In Encyclopedia of Food Sciences and Nutrition (Second Edition); Caballero, B., Ed.; Academic Press: Oxford, 2003; pp 3739-3749.

(106) Scigelova, M.; Hornshaw, M.; Giannakopulos, A.; Makarov, A. Fourier Transform Mass Spectrometry. Mol. Cell. Proteomics MCP 2011, 10 (7).

(107) Karas, M.; Bahr, U.; Gießmann, U. Matrix-Assisted Laser Desorption Ionization Mass Spectrometry. Mass Spectrom. Rev. 10 (5), 335-357.

(108) Hillenkamp, F.; Karas, M. The MALDI Process and Method. In MALDI MS; Hillenkamp, F., Peter-Katalinić, J., Eds.; Wiley-VCH Verlag GmbH \& Co. KGaA, 2007; pp 1-28.

(109) Gross, J. H. Matrix-Assisted Laser Desorption/Ionization. In Mass Spectrometry; Springer, Berlin, Heidelberg, 2011; pp 507-559.

(110) Knochenmuss, R. MALDI and Related Methods: A Solved Problem or Still a Mystery? Mass Spectrom. 2013, 2 (Spec Iss).

(111) Molin, L.; Seraglia, R.; Czarnocki, Z.; Maurin, J. K.; Pluciński, F. A.; Traldi, P. On the Primary Ionization Mechanism(s) in Matrix-Assisted Laser Desorption Ionization https://www.hindawi.com/journals/jamc/2012/161865/ (accessed Jun 18, 2018).

(112) Zenobi, R.; Knochenmuss, R. Ion Formation in MALDI Mass Spectrometry. Mass Spectrom. Rev. 17 (5), 337-366.

(113) Knochenmuss, R. Ion Formation Mechanisms in UV-MALDI. Analyst 2006, 131 (9), 966-986.

(114) Kirmess, K. M.; Knochenmuss, R.; Blanchard, G. J.; Kinsel, G. R. MALDI Ionization Mechanisms Investigated by Comparison of Isomers of Dihydroxybenzoic Acid. J. Mass Spectrom. 51 (1), 79-85.

(115) Knochenmuss, R.; Zenobi, R. MALDI Ionization: The Role of In-Plume Processes. Chem. Rev. 2003, 103 (2), 441-452.

(116) Fuchs, B.; Süß, R.; Schiller, J. An Update of MALDI-TOF Mass Spectrometry in Lipid Research. Prog. Lipid Res. 2010, 49 (4), 450-475.

(117) Cohen, S. L.; Chait, B. T. Influence of Matrix Solution Conditions on the MALDI/MS Analysis of Peptides and Proteins. Anal. Chem. 1996, 68 (1), 31-37.

(118) Schiller, J.; Süß, R.; Arnhold, J.; Fuchs, B.; Leßig, J.; Müller, M.; Petković, M.; Spalteholz, H.; Zschörnig, O.; Arnold, K. Matrix-Assisted Laser Desorption and Ionization Time-of-Flight (MALDI-TOF) Mass Spectrometry in Lipid and Phospholipid Research. Prog. Lipid Res. 2004, 43 (5), 449-488.

(119) Teuber, K.; Schiller, J.; Fuchs, B.; Karas, M.; Jaskolla, T. W. Significant Sensitivity Improvements by Matrix Optimization: A MALDI-TOF Mass 
Spectrometric Study of Lipids from Hen Egg Yolk. Chem. Phys. Lipids 2010, 163 (6), 552-560.

(120) Cerruti, C. D.; Benabdellah, F.; Laprévote, O.; Touboul, D.; Brunelle, A. MALDI Imaging and Structural Analysis of Rat Brain Lipid Negative Ions with 9Aminoacridine Matrix. Anal. Chem. 2012, 84 (5), 2164-2171.

(121) Sun, G.; Yang, K.; Zhao, Z.; Guan, S.; Han, X.; Gross, R. W. Matrix-Assisted Laser Desorption/Ionization-Time of Flight Mass Spectrometric Analysis of Cellular Glycerophospholipids Enabled by Multiplexed Solvent Dependent Analyte-Matrix Interactions. Anal. Chem. 2008, 80 (19), 7576-7585.

(122) Rujoi, M.; Estrada, R.; Yappert, M. C. In Situ MALDI-TOF MS Regional Analysis of Neutral Phospholipids in Lens Tissue. Anal. Chem. 2004, 76 (6), $1657-1663$.

(123) Thomas, A.; Charbonneau, J. L.; Fournaise, E.; Chaurand, P. Sublimation of New Matrix Candidates for High Spatial Resolution Imaging Mass Spectrometry of Lipids: Enhanced Information in Both Positive and Negative Polarities after 1,5Diaminonapthalene Deposition. Anal. Chem. 2012, 84 (4), 2048-2054.

(124) Teuber, K.; Schiller, J.; Fuchs, B.; Karas, M.; Jaskolla, T. W. Significant Sensitivity Improvements by Matrix Optimization: A MALDI-TOF Mass Spectrometric Study of Lipids from Hen Egg Yolk. Chem. Phys. Lipids 2010, 163 (6), 552-560.

(125) Lorkiewicz, P.; Cecilia Yappert, M. 2-(2-Aminoethylamino)-5-Nitropyridine as a Basic Matrix for Negative-Mode Matrix-Assisted Laser Desorption/Ionization Analysis of Phospholipids. J. Mass Spectrom. 2009, 44 (1), 137-143.

(126) Lee, J. W.; Nishiumi, S.; Yoshida, M.; Fukusaki, E.; Bamba, T. Simultaneous Profiling of Polar Lipids by Supercritical Fluid Chromatography/Tandem Mass Spectrometry with Methylation. J. Chromatogr. A 2013, 1279, 98-107.

(127) Fukuyama, Y.; Funakoshi, N.; Takeyama, K.; Hioki, Y.; Nishikaze, T.; Kaneshiro, K.; Kawabata, S.; Iwamoto, S.; Tanaka, K. 3-Aminoquinoline/PCoumaric Acid as a MALDI Matrix for Glycopeptides, Carbohydrates, and Phosphopeptides. Anal. Chem. 2014, 86 (4), 1937-1942.

(128) Hsu, J.; Chang, S. J.; Franz, A. H. MALDI-TOF and ESI-MS Analysis of Oligosaccharides Labeled with a New Multifunctional Oligosaccharide Tag. $J$. Am. Soc. Mass Spectrom. 2006, 17 (2), 194-204.

(129) Yang, H. J.; Lee, A.; Lee, M. K.; Kim, W.; Kim, J. Detection of Small Neutral Carbohydrates Using Various Supporting Materials in Laser Desorption/Ionization Mass Spectrometry. Bull. Korean Chem. Soc. 2010, 31 (1), $35-40$.

(130) Guilhaus, M. Special Feature: Tutorial. Principles and Instrumentation in Timeof-Flight Mass Spectrometry. Physical and Instrumental Concepts. J. Mass Spectrom. 30 (11), 1519-1532.

(131) Ingle, J.; Crouch, S. Spectrochemical Analysis; Prentice Hall: Englewood Cliffs, NJ, 1988.

(132) Kaufmann Raimund; Chaurand Pierre; Kirsch Dieter; Spengler Bernhard. PostSource Decay and Delayed Extraction in Matrix-Assisted Laser Desorption/Ionization-Reflectron Time-of-Flight Mass Spectrometry. Are There Trade-Offs? Rapid Commun. Mass Spectrom. 1996, 10 (10), 1199-1208. 
(133) Jacobsen, N. NMR Spectroscopy Explained; Wiley Interscience: Hoboken, NJ, 2007.

(134) Engel, T.; Hehre, W. Quantum Chemistry and Spectroscopy, 3rd ed.; Prentice Hall, 2010.

(135) Kroon, J.; Kroon-Batenburg, L. M. J.; Leeflang, B. R.; Vliegenthart, J. F. G. Intramolecular versus Intermolecular Hydrogen Bonding in Solution. J. Mol. Struct. 1994, 322, 27-31.

(136) Zuiderweg, E. Mapping Protein-Protein Interactions in Solution by NMR Spectroscopy - Biochemistry (ACS Publications). Am. Chem. Soc. 2002, 41 (1).

(137) Fernández-Alonso, M. del C.; Díaz, D.; Berbis, M. Á.; Marcelo, F.; Cañada, J.; Jiménez-Barbero, J. Protein-Carbohydrate Interactions Studied by NMR: From Molecular Recognition to Drug Design. Curr. Protein Pept. Sci. 2012, 13 (8), 816-830.

(138) Hoffmann, E. de; Stroobant, V. Mass Spectrometry: Principles and Applications; John Wiley \& Sons, 2007.

(139) Steven, R. T.; Race, A. M.; Bunch, J. Para-Nitroaniline Is a Promising Matrix for MALDI-MS Imaging on Intermediate Pressure MS Systems. J. Am. Soc. Mass Spectrom. 2013, 24 (5), 801-804.

(140) Dueñas, M. E.; Carlucci, L.; Lee, Y. J. Matrix Recrystallization for MALDI-MS Imaging of Maize Lipids at High-Spatial Resolution. J. Am. Soc. Mass Spectrom. 2016, 27 (9), 1575-1578.

(141) Bligh, E. G.; Dyer, W. J. A Rapid Method of Total Lipid Extraction and Purification. Can. J. Biochem. Physiol. 1959, 37 (1), 911-917.

(142) Byrdwell, W. C.; Sato, H.; Schwarz, A. K.; Borchman, D.; Yappert, M. C.; Tang, D. 31P NMR Quantification and Monophasic Solvent Purification of Human and Bovine Lens Phospholipids. Lipids 2002, 37 (11), 1087-1092.

(143) Keller, B. O.; Sui, J.; Young, A. B.; Whittal, R. M. Interferences and Contaminants Encountered in Modern Mass Spectrometry. Anal. Chim. Acta 2008, 627 (1), 71-81.

(144) Schwarzinger, C.; Gabriel, S.; Beißmann, S.; Buchberger, W. Quantitative Analysis of Polymer Additives with MALDI-TOF MS Using an Internal Standard Approach. J. Am. Soc. Mass Spectrom. 2012, 23 (6), 1120-1125.

(145) Tris(2,4-di-tert-butylphenyl) phosphite 441791 http://www.sigmaaldrich.com/catalog/product/aldrich/441791 (accessed Apr 26, 2017).

(146) Fuchs, B.; Schiller, J.; Süß, R.; Schürenberg, M.; Suckau, D. A Direct and Simple Method of Coupling Matrix-Assisted Laser Desorption and Ionization Time-ofFlight Mass Spectrometry (MALDI-TOF MS) to Thin-Layer Chromatography (TLC) for the Analysis of Phospholipids from Egg Yolk. Anal. Bioanal. Chem. 2007, 389 (3), 827-834.

(147) Pól, J.; Vidová, V.; Hyötyläinen, T.; Volný, M.; Novák, P.; Strohalm, M.; Kostiainen, R.; Havlíček, V.; Wiedmer, S. K.; Holopainen, J. M. Spatial Distribution of Glycerophospholipids in the Ocular Lens. PLOS ONE 2011, 6 (4).

(148) Ellis, S. R.; Wu, C.; Deeley, J. M.; Zhu, X.; Truscott, R. J. W.; Panhuis, M. in het; Cooks, R. G.; Mitchell, T. W.; Blanksby, S. J. Imaging of Human Lens Lipids by 
Desorption Electrospray Ionization Mass Spectrometry. J. Am. Soc. Mass Spectrom. 2010, 21 (12), 2095-2104.

(149) Anderson, D. M. G.; Ablonczy, Z.; Koutalos, Y.; Spraggins, J.; Crouch, R. K.; Caprioli, R. M.; Schey, K. L. High Resolution MALDI Imaging Mass Spectrometry of Retinal Tissue Lipids. J. Am. Soc. Mass Spectrom. 2014, 25 (8), 1394-1403.

(150) Roy, M. C.; Nakanishi, H.; Takahashi, K.; Nakanishi, S.; Kajihara, S.; Hayasaka, T.; Setou, M.; Ogawa, K.; Taguchi, R.; Naito, T. Salamander Retina

Phospholipids and Their Localization by MALDI Imaging Mass Spectrometry at Cellular Size Resolution. J. Lipid Res. 2011, 52 (3), 463-470.

(151) Serna, J.; García-Seisdedos, D.; Alcázar, A.; Lasunción, M. Á.; Busto, R.; Pastor, Ó. Quantitative Lipidomic Analysis of Plasma and Plasma Lipoproteins Using MALDI-TOF Mass Spectrometry. Chem. Phys. Lipids 2015, 189, 7-18.

(152) Nouri-Sorkhabi, M. H.; Agar, N. S.; Sullivan, D. R.; Gallagher, C.; Kuchel, P. W. Phospholipid Composition of Erythrocyte Membranes and Plasma of Mammalian Blood Including Australian Marsupials; Quantitative 31P NMR Analysis Using Detergent. Comp. Biochem. Physiol. B Biochem. Mol. Biol. 1996, 113 (2), 221227.

(153) Zschörnig, O.; Richter, V.; Rassoul, F.; Süß, R.; Arnold, K.; Schiller, D. J. Analysis of Human Blood Plasma by MALDI-TOF MS - Evaluation of Critical Parameters. Anal. Lett. 2006, 39 (6), 1101-1113.

(154) Yin, W.; Carballo-Jane, E.; McLaren, D. G.; Mendoza, V. H.; Gagen, K.; Geoghagen, N. S.; McNamara, L. A.; Gorski, J. N.; Eiermann, G. J.; Petrov, A.; et al. Plasma Lipid Profiling across Species for the Identification of Optimal Animal Models of Human Dyslipidemia. J. Lipid Res. 2012, 53 (1), 51-65.

(155) Hayasaka, T.; Goto-Inoue, N.; Zaima, N.; Shrivas, K.; Kashiwagi, Y.; Yamamoto, M.; Nakamoto, M.; Setou, M. Imaging Mass Spectrometry with Silver Naeoparticles Reveals the Distribution of Fatty Acids in Mouse Retinal Sections. J. Am. Soc. Mass Spectrom. 2010, 21 (8), 1446-1454.

(156) Borchman, D.; Yappert, M. C. Lipids and the Ocular Lens. J. Lipid Res. 2010, 51 (9), 2473-2488.

(157) Shitama, T.; Hayashi, H.; Noge, S.; Uchio, E.; Oshima, K.; Haniu, H.; Takemori, N.; Komori, N.; Matsumoto, H. Proteome Profiling of Vitreoretinal Diseases by Cluster Analysis. PROTEOMICS - Clin. Appl. 2008, 2 (9), 1265-1280.

(158) Bishop, P. N.; Takanosu, M.; le Goff, M.; Mayne, R. The Role of the Posterior Ciliary Body in the Biosynthesis of Vitreous Humour. Eye 2002, 16 (4), 454-460.

(159) Bron, A. J.; Sparrow, J.; Brown, N. a. P.; Harding, J. J.; Blakytny, R. The Lens in Diabetes. Eye 1993, 7 (2), 260-275.

(160) Ishida, S.; Usui, T.; Yamashiro, K.; Kaji, Y.; Ahmed, E.; Carrasquillo, K. G.; Amano, S.; Hida, T.; Oguchi, Y.; Adamis, A. P. VEGF164 Is Proinflammatory in the Diabetic Retina. Invest. Ophthalmol. Vis. Sci. 2003, 44 (5), 2155-2162.

(161) Park, S.-H.; Park, J.-W.; Park, S.-J.; Kim, K.-Y.; Chung, J.-W.; Chun, M.-H.; Oh, S.-J. Apoptotic Death of Photoreceptors in the Streptozotocin-Induced Diabetic Rat Retina. Diabetologia 2003, 46 (9), 1260-1268. 
(162) Sebag, J. Abnormalities of Human Vitreous Structure in Diabetes. Graefes Arch. Clin. Exp. Ophthalmol. Albrecht Von Graefes Arch. Klin. Exp. Ophthalmol. 1993, 231 (5), 257-260.

(163) Schnepf, A.; Yappert, M. C.; Borchman, D. Regional Distribution of Phospholipids in Porcine Vitreous Humor. Exp. Eye Res. 2017, 160, 116-125.

(164) Krause AC. Chemistry of the Vitreous Humor: Iii. Lipids. Arch. Ophthalmol. 1935, 13 (6), 1022-1025.

(165) Yappert, M. C.; Rujoi, M.; Borchman, D.; DuPre, D. B. Dihydrosphingomyelin in Primate Lens Membranes: Correlation with Lens Growth. Invest. Ophthalmol. Vis. Sci. 2003, 44 (13), 4480-4480.

(166) Magness. Biophysical and Bioanalytical Studies of Hyaluronan and Lipids.

(167) Brügger, B.; Erben, G.; Sandhoff, R.; Wieland, F. T.; Lehmann, W. D. Quantitative Analysis of Biological Membrane Lipids at the Low Picomole Level by Nano-Electrospray Ionization Tandem Mass Spectrometry. Proc. Natl. Acad. Sci. U. S. A. 1997, 94 (6), 2339-2344.

(168) Stoeckli, M.; Staab, D.; Schweitzer, A. Compound and Metabolite Distribution Measured by MALDI Mass Spectrometric Imaging in Whole-Body Tissue Sections. Int. J. Mass Spectrom. 2007, 260 (2), 195-202.

(169) Kubo, K.; Nakamura, T.; Takagaki, K.; Yoshida, Y.; Endo, M. Depolymerization of Hyaluronan by Sonication. Glycoconj. J. 1993, 10 (6), 435-439.

(170) Spagnoli, C.; Korniakov, A.; Ulman, A.; Balazs, E. A.; Lyubchenko, Y.; Cowman, M. Hyaluronan Conformations on Surfaces: Effect of Surface Charge and Hydrophobicity - ScienceDirect. Carbohydr. Res. 2005.

(171) Taglienti, A.; Cellesi, F.; Crescenzi, V.; Sequi, P.; Valentini, M.; Tirelli, N. Investigating the Interactions of Hyaluronan Derivatives with Biomolecules. The Use of Diffusional NMR Techniques. Macromol. Biosci. 2006, 6 (8), 611-622.

(172) Klaassen, I.; Van Noorden, C. J. F.; Schlingemann, R. O. Molecular Basis of the Inner Blood-Retinal Barrier and Its Breakdown in Diabetic Macular Edema and Other Pathological Conditions. Prog. Retin. Eye Res. 2013, 34, 19-48.

(173) Deemter, M. van; Pas, H. H.; Kuijer, R.; Worp, R. J. van der; Hooymans, J. M. M.; Los, L. I. Enzymatic Breakdown of Type II Collagen in the Human Vitreous. Invest. Ophthalmol. Vis. Sci. 2009, 50 (10), 4552-4560. 


\section{APPENDIX}

\section{COPYRIGHT STATEMENT}

Chapter 2 includes text and figures from the article " Regional Distribution of Phospholipids in Porcine Vitreous Humor" published in Experimental Eye Research 2017, volume 160, pages 116-125. Permission to reprint this material was granted by Elsevier on July 10, 2018. 


\section{Dear Abigail Schnepf}

We hereby grant you permission to reprint the material detailed below at no charge in your thesis subject to the following conditions:

1. If any part of the material to be used (for example, figures) has appeared in our publication with credit or acknowledgement to another source, permission must also be sought from that source. If such permission is not obtained then that material may not be included in your publication/copies.

2. Suitable acknowledgment to the source must be made, either as a footnote or in a reference list at the end of your publication, as follows:

"This article was published in Publication title, Vol number, Author(s), Title of article, Page Nos, Copyright Elsevier (or appropriate Society name) (Year)."

3. Your thesis may be submitted to your institution in either print or electronic form.

4. Reproduction of this material is confined to the purpose for which permission is hereby given.

5. This permission is granted for non-exclusive world English rights only. For other languages please reapply separately for each one required. Permission excludes use in an electronic form other than submission. Should you have a specific electronic project in mind please reapply for permission

6. This includes permission for UMI to supply single copies, on demand, of the complete thesis. Should your thesis be published commercially, please reapply for permission.

Yours sincerely

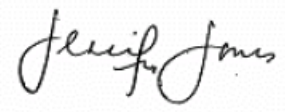

Jennifer Jones

Permissions Specialist

Elsevier Limited, a company registered in England and Wales with company number 1982084, whose registered office is The Boulevard, Langford Lane, Kidlington, Oxford, OX5 1GB, United Kingdom. 


\section{CURRICULUM VITAE}

NAME:

ADDRESS:

DOB:

EDUCATION

\& TRAINING:
Abigail Nicole Schnepf

Department of Chemistry University of Louisville

2320 South Brook Street

Louisville, KY 40292

Indianapolis, Indiana - August $2^{\text {nd }}, 1990$
B.S., Chemistry

Bellarmine University

2008-2012

M.S., Analytical Chemistry

University of Louisville

2013-2016

Ph.D., Analytical Chemistry

University of Louisville

2016-2018

PUBLICATIONS: $\quad$ Schnepf, A.; Yappert, M. C.; Borchman, D. Regional Distribution of Phospholipids in Porcine Vitreous Humor. Exp. Eye Res.

2017, 160, 116-125.

PROFESSIONAL SOCIETIES:

Association for Research in Vision and Opthalmology

\section{NATIONAL MEETING}

PRESENTATIONS: Abigail Schnepf, Douglas Borchman, and M.C. Yappert. 2018. "Mass spectral study of the impact of diabetes in the distribution of glucose and lipids in human vitreous humor" ARVO Annual Meeting, Honolulu, HI.

Abigail Schnepf and M.C. Yappert. 2018. "Impact of hyaluronan on the ionization efficiency of glucose and phospholipids using 
MALDI-MS" ASMS $66^{\text {th }}$ Conference on Mass Spectrometry and Allied Topics, San Diego, CA.

Abigail Schnepf, Douglas Borchman, and M.C. Yappert. 2017. "Cholesterol, phospholipids and glucose detection in vitreous humor from diabetic human donors" ASMS $65^{\text {th }}$ Conference on Mass Spectrometry and Allied Topics, Indianapolis, IN.

Abigail Schnepf, Douglas Borchman, and M.C. Yappert. 2016. "In-situ and in-vitro Regional Analysis of Lipids in Mammalian Vitreous Humor using MALDI-MS" $64^{\text {th }}$ ASMS Conference on Mass Spectrometry and Allied Topics, San Antonio, TX.

Abigail Schnepf, Douglas Borchman, and M.C. Yappert. 2015. "Imaging of lipids in the ocular vitreous humor using matrixassisted laser desorption ionization-mass spectrometry" The Southeastern Regional Meeting of the American Chemical Society, Memphis, TN.

ACTIVITIES: Chemistry Graduate Student Association Treasurer 2014-2017 